\title{
Development of an enantioselective synthesis of (-)-euonyminol.
}

\author{
Martin Tomanik, ${ }^{1}$ Zhi Xu, ${ }^{1}$ Facheng Guo, ${ }^{1,2}$ Zechun Wang, ${ }^{1}$ Ke R. Yang, ${ }^{1,2}$ Victor S. Batista, ${ }^{1,2}$ \\ and Seth B. Herzon $1,3, *$
}

${ }^{1}$ Department of Chemistry, Yale University, New Haven, Connecticut 06520, United States.

${ }^{2}$ Energy Sciences Institute, Yale University, West Haven, Connecticut 06516, United States.

${ }^{3}$ Department of Pharmacology, Yale School of Medicine, New Haven, Connecticut 06520, United States.

The Journal of Organic Chemistry.

Supporting Information

\section{Index:}

Complete set of reaction conditions described in Table 3 S1

Comparison of ${ }^{1} \mathrm{H}$ NMR data of synthetic euonyminol (1)

Comparison of ${ }^{13} \mathrm{C}$ NMR data of synthetic and calculated euonyminol (1).................................S4

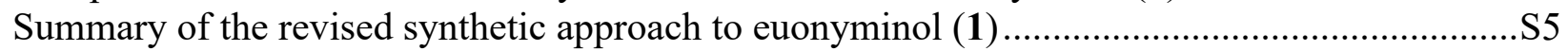

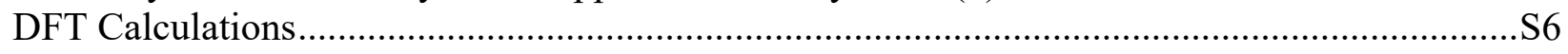

Catalog of nuclear magnetic resonance spectra.......................................................................S33

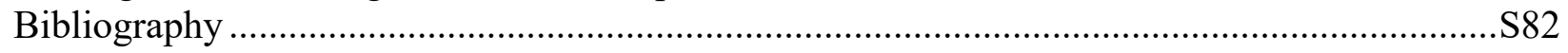




\section{Complete set of reaction conditions described in Table 3.}

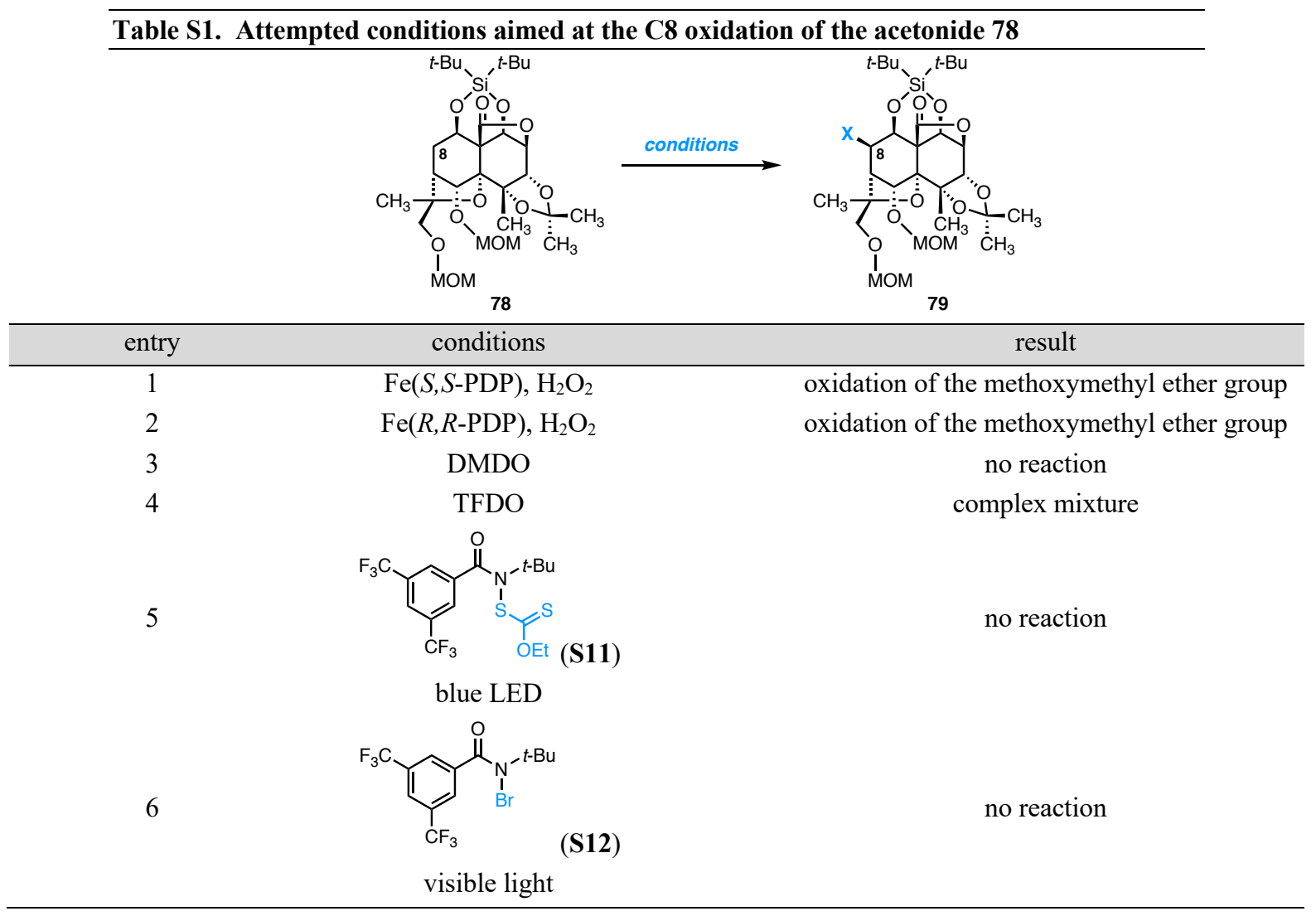

Entry 1: A solution of $\quad\left(2 S, 2^{\prime} S-(-)-\left[N, N^{\prime}-\right.\right.$ bis(2-pyridylmethyl) $]-2,2^{\prime}-$ bipyrrolidinebis(acetonitrile)iron(II) hexafluoroantimonate $(2.4 \mathrm{mg}, 2.60 \mu \mathrm{mol}, 0.25$ equiv) in acetonitrile $(150 \mu \mathrm{L})$ and a solution of aqueous hydrogen peroxide $\left(50 \mathrm{wt} \%\right.$ in $\mathrm{H}_{2} \mathrm{O}, 3.60 \mu \mathrm{L}, 51.2$ $\mu \mathrm{mol}, 5.0$ equiv) in in acetonitrile $(100 \mu \mathrm{L})$ were added via two syringe pumps over $1 \mathrm{~h}$ simultaneously to a solution of the lactone $\mathbf{7 8}(6.3 \mathrm{mg}, 10.2 \mu \mathrm{mol}, 1$ equiv) and acetic acid (1.0 $\mu \mathrm{L}, 5.10 \mu \mathrm{mol}, 0.50$ equiv) in acetonitrile $(200 \mu \mathrm{L})$ at $23{ }^{\circ} \mathrm{C}$. The reaction was monitored by LC/MS analysis, which indicated oxidation of the methoxymethyl ether group and subsequent decomposition.

Entry 2: $\quad$ A $\quad$ solution of $\quad\left(2 R, 2^{\prime} R-(-)-\left[N, N^{\prime}\right.\right.$-bis(2-pyridylmethyl) $]-2,2^{\prime}-$ bipyrrolidinebis(acetonitrile)iron(II) hexafluoroantimonate $(2.7 \mathrm{mg}, 2.90 \mu \mathrm{mol}, 0.25$ equiv) in acetonitrile $(160 \mu \mathrm{L})$ and a solution of aqueous hydrogen peroxide $\left(50 \mathrm{wt} \%\right.$ in $\mathrm{H}_{2} \mathrm{O}, 4.00 \mu \mathrm{L}, 58.6$ $\mu \mathrm{mol}, 5.0$ equiv) in in acetonitrile $(110 \mu \mathrm{L})$ were added via two syringe pumps over $1 \mathrm{~h}$ simultaneously to a solution of the lactone $78(7.2 \mathrm{mg}, 11.7 \mu \mathrm{mol}, 1$ equiv) and acetic acid (1.0 $\mu \mathrm{L}, 5.90 \mu \mathrm{mol}, 0.50$ equiv) in acetonitrile $(200 \mu \mathrm{L})$ at $23{ }^{\circ} \mathrm{C}$. The reaction was monitored by LC/MS analysis, which indicated oxidation of the methoxymethyl ether group and subsequent decomposition. 
Entry 3: A solution of dimethyldioxirane in acetone ( $60 \mathrm{mM}, 340 \mu \mathrm{L}, 20.3 \mu \mathrm{mol}, 2.50$ equiv) was added to a solution of the lactone $78\left(5.0 \mathrm{mg}, 8.10 \mu \mathrm{mol}, 1\right.$ equiv) in acetone $(200 \mu \mathrm{L})$ at $-78^{\circ} \mathrm{C}$. The reaction mixture was stirred for $30 \mathrm{~min}$ at $-78^{\circ} \mathrm{C}$. The product mixture was then warmed to $-40{ }^{\circ} \mathrm{C}$ over $30 \mathrm{~min}$. The product mixture was then warmed to $0{ }^{\circ} \mathrm{C}$ over $30 \mathrm{~min}$. The reaction was monitored by LC/MS analysis, which indicated no reaction.

Entry 4: A solution of methyl(trifluoromethyl)dioxirane in trifluoroacetone ( $\sim .5 \mathrm{M}, 50.0 \mu \mathrm{L}, 24.4$ $\mu \mathrm{mol}, 2.50$ equiv) was added dropwise via a cold glass pipette to a solution of the lactone 101 (5.0 $\mathrm{mg}, 9.80 \mu \mathrm{mol}, 1$ equiv) in dichloromethane $(200 \mu \mathrm{L})$ at $-78^{\circ} \mathrm{C}$. The reaction mixture was stirred for $30 \mathrm{~min}$ at $-78^{\circ} \mathrm{C}$. The product mixture was then warmed to $-40^{\circ} \mathrm{C}$ over $30 \mathrm{~min}$. The product mixture was then warmed to $0{ }^{\circ} \mathrm{C}$ over $30 \mathrm{~min}$. The reaction was monitored by LC/MS analysis, which indicated formation of a complex mixture of products.

Entry 5: The xanthylamide $\mathbf{S 1 1}{ }^{17 \mathrm{a}}$ (4.7 $\mathrm{mg}, 10.7 \mu \mathrm{mol}, 1.10$ equiv), the lactone $\mathbf{7 8}(6.0 \mathrm{mg}, 9.80$ $\mu \mathrm{mol}, 1$ equiv), and trifluorotoluene $(200 \mu \mathrm{L})$ were combined in a 1 -dram vial in a nitrogen-filled glovebox. The reaction vial was sealed and the sealed reaction vial was removed from the glovebox. The reaction vial was suspended above a stir plate and next to a Kessil Blue LED light such that the LED lamp was $1.0 \mathrm{~cm}$ away from the reaction vial. The reaction vial, the Kessil Blue LED lamp, and the stir plate were covered with an aluminum foil. The covered reaction vial was irradiated and stirred for $24 \mathrm{~h}$ at $23{ }^{\circ} \mathrm{C}$. The reaction was monitored by LC/MS analysis, which indicated no reaction.

Entry 6: The bromoamide $\mathbf{S 1 2}{ }^{17 \mathrm{~b}}$ (3.5 $\mathrm{mg}, 8.90 \mu \mathrm{mol}, 1.10$ equiv), the lactone $\mathbf{7 8}$ (5.0 $\mathrm{mg}, 8.10$ $\mu \mathrm{mol}, 1$ equiv), and benzene $(200 \mu \mathrm{L})$ were combined in a 1-dram vial in a nitrogen-filled glovebox. The reaction vial was sealed and the sealed reaction vial was removed from the glovebox. The reaction vial was suspended above a stir plate and next to a $100 \mathrm{~W}$ tungsten lightbulb such that the light source was $1.0 \mathrm{~cm}$ away from the reaction vial. The reaction vial, the light source, and the stir plate were covered with an aluminum foil. The covered reaction vial was irradiated and stirred for $24 \mathrm{~h}$ at $23{ }^{\circ} \mathrm{C}$. The reaction was monitored by LC/MS analysis, which indicated no reaction. 


\section{Comparison of ${ }^{1} \mathrm{H}$ NMR spectroscopic data of synthetic euonyminol (1).}

\begin{tabular}{|c|c|c|c|}
\hline \multicolumn{4}{|c|}{$\begin{array}{l}\text { Table S2. Comparison of synthetic }{ }^{1} \mathrm{H} \text { NMR data of euonyminol (1) from the White } \\
\text { and Herzon laboratories. }\end{array}$} \\
\hline & 13 & $\begin{array}{l}\mathrm{I}_{\mathrm{CH}} \mathrm{OH}_{3} \\
14 \\
\text { nol (1) } \\
\end{array}$ & \\
\hline \multirow[t]{2}{*}{ position } & $\begin{array}{l}\text { synthetic }(-)-1^{1} \\
{\left[500 \mathrm{MHz}, \mathrm{D}_{2} \mathrm{O}\right]}\end{array}$ & $\begin{array}{c}\text { synthetic }( \pm)-\mathbf{1}^{2} \\
{\left[\mathrm{D}_{2} \mathrm{O}\right]}\end{array}$ & \\
\hline & ${ }^{1} \mathrm{H}[\delta \mathrm{H}(\mathrm{ppm})$, mult, $\mathrm{J}(\mathrm{Hz})]$ & ${ }^{1} \mathrm{H}[\delta \mathrm{H}(\mathrm{ppm})$, mult, $\mathrm{J}(\mathrm{Hz})]$ & $\operatorname{abs}(\Delta \delta)$ \\
\hline $\mathrm{H}-1$ & $4.33(\mathrm{~d}, 3.5)$ & $4.30(\mathrm{~m})$ & 0.03 \\
\hline $\mathrm{H}-2$ & $4.03(t, 3.2)$ & $4.00(\mathrm{~m})$ & 0.03 \\
\hline $\mathrm{H}-3$ & $3.66(\mathrm{~d}, 2.9)$ & $3.65(\mathrm{~d}, 4)$ & 0.01 \\
\hline H-6 & $5.22(\mathrm{~s})$ & $5.10(\mathrm{~s})$ & 0.02 \\
\hline $\mathrm{H}-7$ & $2.39(\mathrm{~d}, 4.0)$ & $2.30(\mathrm{~s})$ & 0.09 \\
\hline H-8 & $4.21(\mathrm{dd}, 5.3,4.1)$ & $4.30(\mathrm{~m})$ & 0.09 \\
\hline H-9 & $4.37(\mathrm{~d}, 5.4)$ & $4.30(\mathrm{~m})$ & 0.07 \\
\hline $\mathrm{H}-12_{\mathrm{a}}$ & $3.92(\mathrm{~d}, 11.4)$ & $3.95(\mathrm{~m})$ & 0.03 \\
\hline $\mathrm{H}-12 \mathrm{~b}$ & $3.73(\mathrm{~d}, 11.4)$ & $3.68(\mathrm{~d}, 11)$ & 0.05 \\
\hline $\mathrm{H}-13$ & $1.44(\mathrm{~s})$ & $1.36(\mathrm{~s})$ & 0.08 \\
\hline $\mathrm{H}-14$ & $1.74(\mathrm{~s})$ & $1.58(\mathrm{~s})$ & 0.16 \\
\hline $\mathrm{H}-15_{\mathrm{a}}$ & $4.43(\mathrm{~d}, 13.1)$ & $4.30(\mathrm{~m})$ & 0.13 \\
\hline $\mathrm{H}-15_{b}$ & $4.09(\mathrm{~d}, 13.1)$ & $4.09(\mathrm{~d}, 11)$ & 0.00 \\
\hline
\end{tabular}


Comparison of ${ }^{13} \mathrm{C}$ NMR spectroscopic data of synthetic calculated euonyminol (1).

Table S3. Comparison of synthetic ${ }^{1} \mathrm{H}$ NMR data of euonyminol (1) from the White and Herzon laboratories.

\begin{tabular}{|c|c|c|c|}
\hline & $13 \mathrm{C}$ & 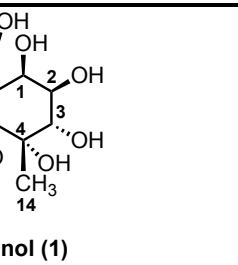 & \\
\hline position & $\begin{array}{c}\text { synthetic (-)-3 } \\
{\left[500 \mathrm{MHz}, \mathrm{CD}_{3} \mathrm{OD}\right]}\end{array}$ & Calculated & \\
\hline & ${ }^{13} \mathrm{C}[\delta \mathrm{C}(\mathrm{ppm})]$ & ${ }^{13} \mathrm{C}[\delta \mathrm{C}(\mathrm{ppm})]$ & abs(exp-calc) \\
\hline C-1 & 74.97 & 77.9 & 2.93 \\
\hline C-2 & 75.81 & 75.4 & 0.41 \\
\hline C-3 & 79.32 & 77.7 & 1.62 \\
\hline C-4 & 73.36 & 73.3 & 0.06 \\
\hline C-5 & 94.50 & 95.5 & 1.00 \\
\hline C-6 & 74.97 & 73.7 & 1.27 \\
\hline C-7 & 53.92 & 50.1 & 3.82 \\
\hline C- 8 & 70.98 & 71.2 & 0.22 \\
\hline C-9 & 76.86 & 79.3 & 2.44 \\
\hline C- 10 & 54.96 & 50.4 & 4.56 \\
\hline C-11 & 85.92 & 87.9 & 1.98 \\
\hline C- 12 & 68.89 & 73.2 & 4.31 \\
\hline C-13 & 18.89 & 21.8 & 2.91 \\
\hline C-14 & 25.85 & 26.6 & 0.75 \\
\hline C- 15 & 59.60 & 65.1 & 5.50 \\
\hline
\end{tabular}




\section{Summary of the revised synthetic approach to euonyminol (1).}
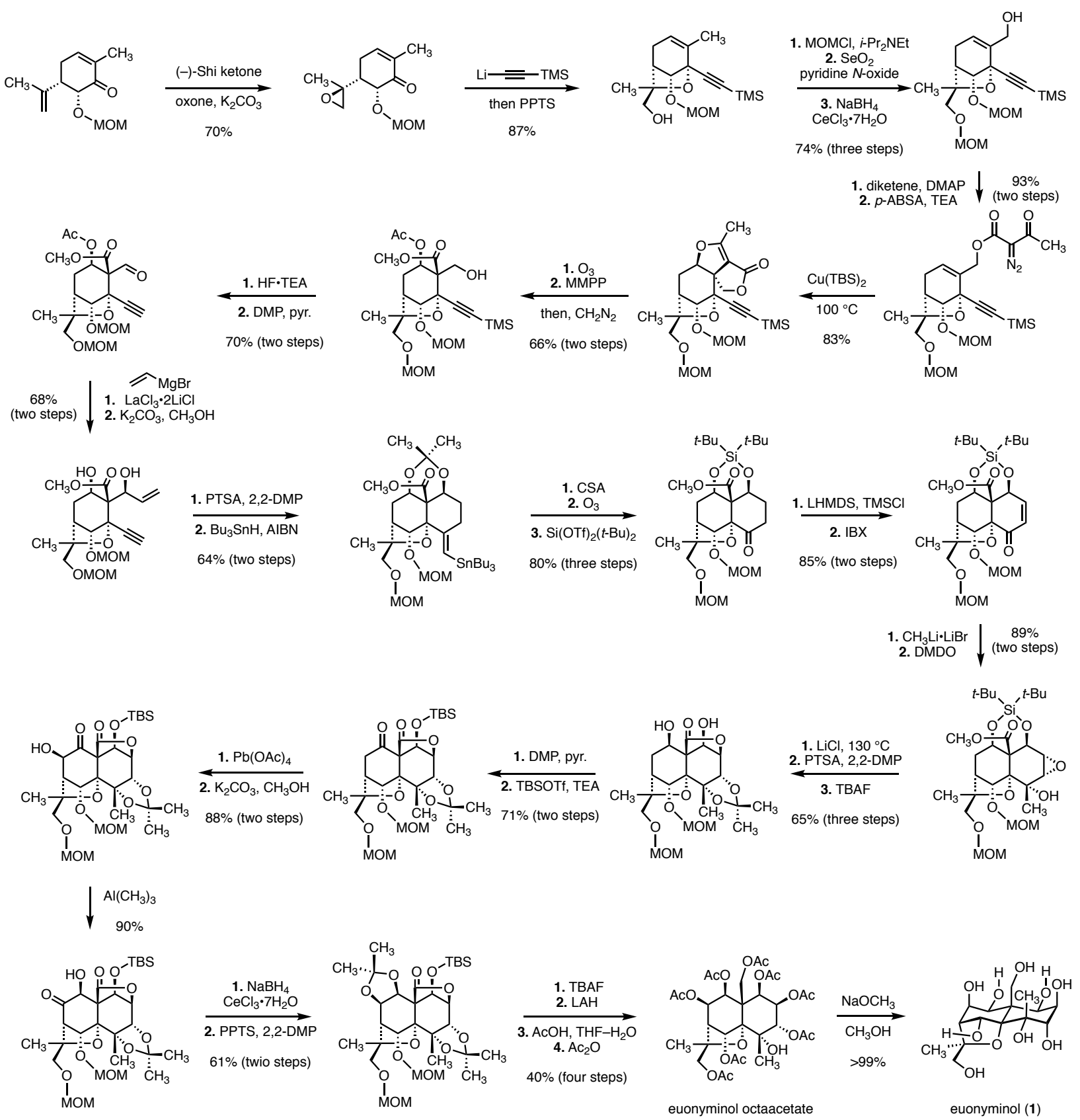


\section{DFT Calculations.}

Radical cyclization calculations of 71:

Density functional theory calculations were performed by Gaussian 2016 software package, revision A.03. ${ }^{3}$ All geometry optimizations, including transition state optimizations, were achieved by (U)B3LYP functional ${ }^{4-5}$ with Def2SVP basis sets ${ }^{6}$ for tin element, and 6-31G(d) basis sets ${ }^{7-8}$ for hydrogen, carbon, and oxygen elements. Single point energy calculations were achieved by (U)B3LYP functional with Def2TZVP basis sets for tin element, and 6-311+G(2df,p) basis sets ${ }^{9-}$ 18 for hydrogen, carbon, and oxygen elements. Frequency calculations were performed with transition state optimizations for inspection purpose. All calculations were performed with D3 Grimme's empirical dispersion. ${ }^{19}$

Table S4. Summary of energies for the optimized structures and transition states.

\begin{tabular}{ccccc}
\hline entry & $\begin{array}{c}\text { compound/ } \\
\text { transition state }\end{array}$ & $\begin{array}{c}\text { absolute energy } \\
\text { (Hartree) }\end{array}$ & $\begin{array}{c}\text { relative energy } \\
(\mathrm{kcal} / \mathrm{mol})\end{array}$ & $\begin{array}{c}\text { Imaginary Frequency } \\
\text { Number }\end{array}$ \\
\hline 1 & $\mathbf{7 1}$ & -1908.53049 & 0.00 & Not Applicable \\
2 & $\mathbf{7 2}$ & -1908.53797 & -4.68 & Not Applicable \\
3 & $\mathbf{7 3}$ & -1908.54384 & -8.38 & Not Applicable \\
4 & $\mathbf{7 4}$ & -1908.56517 & -21.76 & Not Applicable \\
5 & TS-1 & -1908.51710 & 8.41 & 1 \\
6 & $\mathbf{T S - 2}$ & -1908.51604 & 9.07 & 1 \\
7 & $\mathbf{T S}-3$ & -1908.51107 & 12.19 & 1 \\
8 & $\mathbf{7 5}$ & -1908.52533 & 3.24 & Not Applicable \\
9 & $\mathbf{7 6}$ & -1908.53327 & -1.74 & Not Applicable \\
10 & TS-4 & -1908.51920 & 7.09 & 1 \\
11 & TS-5 & -1908.50433 & 16.42 & 1 \\
12 & TS-6 & -1908.52812 & 1.49 & 1 \\
13 & TS-7 & -1908.52322 & 4.56 & 1 \\
\hline
\end{tabular}


Cartesian coordinates for optimized structures and transition states:

Compound 71:

C $3.451900-1.537600 \quad-0.165500$

C $\quad 4.447100 \quad 0.939600 \quad-0.174300$

C $\quad 4.187800 \quad-0.406900 \quad 0.615200$

$\begin{array}{llll}\text { C } & 5.598400 & -1.008400 & 0.894800\end{array}$

C $\quad 6.595800 \quad-0.017500 \quad 1.508400$

$\begin{array}{llll}\text { C } & 6.590300 & 1.332900 & 0.745700\end{array}$

$\begin{array}{llll}\text { C } & 5.155700 & 1.883400 & 0.843500\end{array}$

O $\quad 5.445800 \quad 0.690400 \quad-1.209800$

C $\quad 6.757500 \quad 1.164400 \quad-0.782100$

C $\quad 7.829500 \quad 0.175600 \quad-1.244600$

C $\quad 7.000700 \quad 2.497900-1.523600$

O $\quad 8.299700 \quad 3.014500 \quad-1.201700$

C $\quad 8.345200 \quad 4.305600 \quad-0.712700$

$\begin{array}{llll}\text { O } & 7.991700 & 4.325600 & 0.662500\end{array}$

C $\quad 8.086000 \quad 5.621500 \quad 1.219500$

C $\quad 3.266800 \quad 1.542400 \quad-0.809600$

C $2.902400 \quad 2.126800 \quad-1.916800$

O $\quad 5.517600 \quad-2.170200 \quad 1.700300$

O $\quad 3.443700 \quad-2.692000 \quad 0.691200$

C $4.702500 \quad-3.197700 \quad 1.144700$

C $\quad 4.365600 \quad-4.121300 \quad 2.306000$

C $\quad 5.430200 \quad-3.933600 \quad 0.009800$

$\mathrm{H} \quad 7.326800 \quad 2.025900 \quad 1.157000$

H $\quad 5.972100 \quad-1.291300 \quad-0.092300$

Sn $\quad 0.832900 \quad 2.751900 \quad-2.103800$

C $\quad-0.166800 \quad 2.065500 \quad-0.295000$

C $\quad 0.754200 \quad 4.930400 \quad-2.246400$

C $\quad-0.040800 \quad 1.839700 \quad-3.883700$

C $\quad 3.454400 \quad-0.102600 \quad 1.928800$

$\begin{array}{llll}\text { O } & 2.255300 & 0.483900 & 1.703600\end{array}$

O $\quad 3.880900 \quad-0.289300 \quad 3.046800$

C $\quad 1.567300 \quad 0.937300 \quad 2.884100$

H $\quad 4.037600 \quad-1.716500 \quad-1.078500$

C $\quad 2.012100 \quad-1.338500 \quad-0.550800$

H $\quad 7.591500 \quad-0.472600 \quad 1.496800$

$\begin{array}{llll}\mathrm{H} & 6.327500 & 0.147300 & 2.555400\end{array}$

$\mathrm{H} \quad 4.748300 \quad 1.763900 \quad 1.847500$

H $\quad 7.727400 \quad 0.012800 \quad-2.322900$

H $\quad 8.816900 \quad 0.606000 \quad-1.054500$

H $\quad 7.771600 \quad-0.795000 \quad-0.750800$

$\mathrm{H} \quad 6.972200 \quad 2.292300 \quad-2.601400$

H $\quad 6.220100 \quad 3.215200 \quad-1.276300$

H $9.383200 \quad 4.652100 \quad-0.834500$ 


$\begin{array}{rrrr}\mathrm{H} & 7.670200 & 4.981900 & -1.271200 \\ \mathrm{H} & 9.109400 & 6.024300 & 1.148000 \\ \mathrm{H} & 7.812400 & 5.540500 & 2.274100 \\ \mathrm{H} & 7.399700 & 6.331100 & 0.728100 \\ \mathrm{H} & 3.609600 & 2.280600 & -2.741100 \\ \mathrm{H} & 5.283500 & -4.544900 & 2.723500 \\ \mathrm{H} & 3.858400 & -3.537300 & 3.077700 \\ \mathrm{H} & 3.711600 & -4.930600 & 1.969500 \\ \mathrm{H} & 5.664000 & -3.274200 & -0.830600 \\ \mathrm{H} & 6.369100 & -4.350100 & 0.386300 \\ \mathrm{H} & 4.800100 & -4.745900 & -0.363800 \\ \mathrm{H} & -1.208800 & 1.798700 & -0.493100 \\ \mathrm{H} & -0.140800 & 2.846800 & 0.470700 \\ \mathrm{H} & -0.282600 & 5.276000 & -2.304700 \\ \mathrm{H} & 1.290100 & 5.277200 & -3.134900 \\ \mathrm{H} & 0.030600 & 0.750600 & -3.810900 \\ \mathrm{H} & -1.094500 & 2.119300 & -3.980000 \\ \mathrm{H} & 0.634200 & 1.369900 & 2.523300 \\ \mathrm{H} & 2.172200 & 1.689200 & 3.398600 \\ \mathrm{H} & 1.375400 & 0.101900 & 3.561900 \\ \mathrm{C} & 1.589500 & -1.248400 & -1.810600 \\ \mathrm{H} & 2.279900 & -1.264400 & -2.650700 \\ \mathrm{H} & 0.535700 & -1.128800 & -2.048600 \\ \mathrm{H} & 1.308500 & -1.314900 & 0.275300 \\ \mathrm{H} & 0.375500 & 1.190600 & 0.070400 \\ \mathrm{H} & 1.223200 & 5.371300 & -1.361200 \\ \mathrm{H} & 0.492200 & 2.166500 & -4.781600 \\ \mathrm{O} & 5.001700 & 3.246400 & 0.470600 \\ \mathrm{C} & 4.955600 & 4.127800 & 1.566700 \\ \mathrm{O} & 3.812700 & 3.966600 & 2.364200 \\ \mathrm{H} & 5.809300 & 3.979100 & 2.236900 \\ \mathrm{H} & 4.993900 & 5.130700 & 1.118200 \\ \mathrm{C} & 2.593100 & 4.166300 & 1.655200 \\ \mathrm{H} & 1.795700 & 4.173000 & 2.402400 \\ \mathrm{H} & 2.416100 & 3.362200 & 0.933300 \\ \mathrm{H} & 2.595100 & 5.129600 & 1.121100 \\ & & & \end{array}$

Tomanik et al. "Development of an enantioselective synthesis of (-)-euonyminol." J. Org. Chem. 
Compound 72:

\begin{tabular}{|c|c|c|c|}
\hline & & & \\
\hline & & 900 & \\
\hline & 4.305400 & -0.385500 & 2000 \\
\hline & 5.655600 & & \\
\hline & 800 & 0300 & \\
\hline & 537400 & 26900 & 81 \\
\hline & 082900 & 5400 & 20 \\
\hline & 5.331400 & 0.879800 & -1.1958 \\
\hline & 0.00 & & \\
\hline & 100 & & \\
\hline & 6.927200 & 2.664100 & -1.391200 \\
\hline & 8.251300 & 3.106900 & 5600 \\
\hline & 8.3 & 400 & -0 . \\
\hline & 7.9 & & \\
\hline & 8.06 & & \\
\hline & 2.98 & & -0. \\
\hline & 2.45 & & \\
\hline & 5.4 & -2 & \\
\hline & 3.23 & -2.4 & \\
\hline & 4.4 & & \\
\hline & 4.00 & & \\
\hline & $5.0^{\circ}$ & -3 & \\
\hline & 7.2 & & \\
\hline & 6.01 & -1.2 & -0. \\
\hline $\mathrm{sn}$ & 0.5 & & \\
\hline & -0.9 & & \\
\hline & 0.66 & & \\
\hline & -0.00 & & \\
\hline & 3.6 & -0.2 & \\
\hline $\mathrm{O}$ & 2.5 & & \\
\hline & & & \\
\hline & 1.90 & & \\
\hline & 4.1 & -1. & \\
\hline & 2.34 & -0.315700 & -0.8 \\
\hline $\mathrm{H}$ & & & \\
\hline & & & \\
\hline & 4.70 & 00 & \\
\hline & & & \\
\hline $\mathrm{H}$ & 8.71 & 000 & 300 \\
\hline $\mathrm{H}$ & & & \\
\hline & & & \\
\hline & & & \\
\hline $\mathrm{H}$ & 9.415800 & 4.672400 & -0.629100 \\
\hline & $7.74^{\prime}$ & 5.110400 & -1.13 \\
\hline & 010440 & 606600 & 1.354 \\
\hline
\end{tabular}

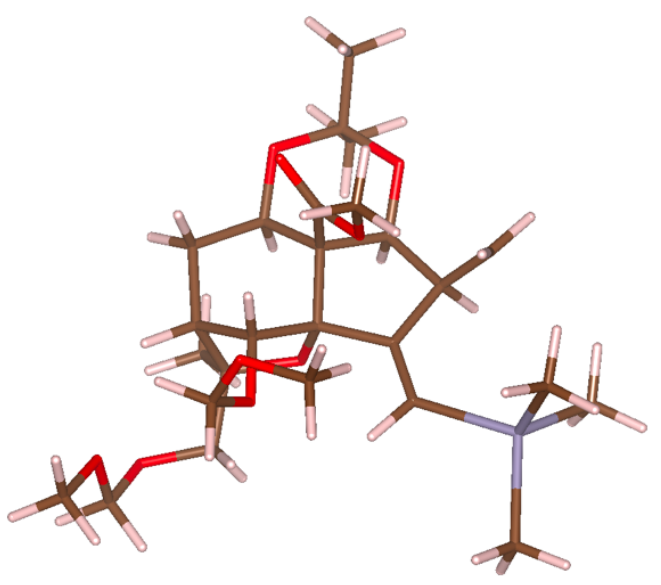

Tomanik et al. "Development of an enantioselective synthesis of (-)-euonyminol." J. Org. Chem. 


$\begin{array}{rrrr}\mathrm{H} & 7.730500 & 5.637600 & 2.408900 \\ \mathrm{H} & 7.434300 & 6.449500 & 0.847200 \\ \mathrm{H} & 3.066200 & 3.145400 & -0.943200 \\ \mathrm{H} & 4.857100 & -4.714400 & 2.187900 \\ \mathrm{H} & 3.592900 & -3.555700 & 2.665200 \\ \mathrm{H} & 3.239600 & -4.804800 & 1.436700 \\ \mathrm{H} & 5.410500 & -3.159800 & -1.222100 \\ \mathrm{H} & 5.936400 & -4.453200 & -0.135800 \\ \mathrm{H} & 4.333500 & -4.533100 & -0.915500 \\ \mathrm{H} & -0.625100 & 3.474200 & 0.341300 \\ \mathrm{H} & -1.173100 & 1.811000 & 0.028400 \\ \mathrm{H} & 0.971200 & 5.267200 & -2.426800 \\ \mathrm{H} & -0.304100 & 4.726200 & -3.538700 \\ \mathrm{H} & -0.905300 & 1.211400 & -3.931700 \\ \mathrm{H} & 0.813100 & 0.770400 & -4.058100 \\ \mathrm{H} & 1.002500 & 1.380900 & 2.989300 \\ \mathrm{H} & 2.576000 & 1.424100 & 3.851300 \\ \mathrm{H} & 1.658800 & -0.105400 & 3.760500 \\ \mathrm{H} & 2.235700 & -0.530700 & -1.902200 \\ \mathrm{C} & 1.004500 & -0.503900 & -0.206900 \\ \mathrm{H} & 0.455800 & -1.417900 & -0.410700 \\ \mathrm{H} & 0.632100 & 0.166100 & 0.554600 \\ \mathrm{H} & -1.923100 & 3.173900 & -0.836400 \\ \mathrm{H} & 1.399900 & 4.375500 & -3.902400 \\ \mathrm{H} & -0.199000 & 0.045900 & -2.785200 \\ \mathrm{O} & 4.920400 & 3.269500 & 0.641300 \\ \mathrm{C} & 4.993100 & 4.092000 & 1.784900 \\ \mathrm{O} & 3.948800 & 3.876700 & 2.695800 \\ \mathrm{H} & 5.917200 & 3.912300 & 2.343100 \\ \mathrm{H} & 4.978700 & 5.117900 & 1.390600 \\ \mathrm{C} & 2.653000 & 4.103300 & 2.147800 \\ \mathrm{H} & 1.953300 & 4.082100 & 2.987000 \\ \mathrm{H} & 2.382800 & 3.327500 & 1.423300 \\ \mathrm{H} & 2.595200 & 5.087300 & 1.656600\end{array}$

Tomanik et al. "Development of an enantioselective synthesis of (-)-euonyminol." J. Org. Chem. 
Compound 73:

$\begin{array}{lrrr}\mathrm{C} & 3.547900 & -1.275800 & -0.154800 \\ \mathrm{C} & 4.499700 & 0.917000 & -0.125600 \\ \mathrm{C} & 4.360700 & -0.356400 & 0.727700 \\ \mathrm{C} & 5.689200 & -1.039200 & 0.963400 \\ \mathrm{C} & 6.686800 & -0.035100 & 1.571300 \\ \mathrm{C} & 6.628300 & 1.314300 & 0.760700 \\ \mathrm{C} & 5.182500 & 1.914900 & 0.818600 \\ \mathrm{O} & 5.462400 & 0.704900 & -1.196800 \\ \mathrm{C} & 6.795600 & 1.115300 & -0.766200 \\ \mathrm{C} & 7.836200 & 0.089200 & -1.225900 \\ \mathrm{C} & 7.103000 & 2.431200 & -1.517000 \\ \mathrm{O} & 8.427400 & 2.876700 & -1.186200 \\ \mathrm{C} & 8.545700 & 4.198900 & -0.807600 \\ \mathrm{O} & 8.091200 & 4.368000 & 0.527500 \\ \mathrm{C} & 8.226400 & 5.703000 & 0.973500 \\ \mathrm{C} & 3.110200 & 1.021500 & -0.765800 \\ \mathrm{C} & 2.614100 & 2.138500 & -1.305200 \\ \mathrm{O} & 5.424600 & -2.236000 & 1.698900 \\ \mathrm{O} & 3.242100 & -2.434700 & 0.603600 \\ \mathrm{C} & 4.441600 & -3.107400 & 1.100000 \\ \mathrm{C} & 3.946600 & -3.978100 & 2.242900 \\ \mathrm{C} & 5.076200 & -3.923300 & -0.032500 \\ \mathrm{H} & 7.368800 & 2.016600 & 1.149900 \\ \mathrm{H} & 6.064500 & -1.322600 & -0.021800 \\ \mathrm{~S} n & 0.597200 & 2.415200 & -2.031600 \\ \mathrm{C} & -0.769300 & 1.302300 & -0.743500 \\ \mathrm{C} & 0.216100 & 4.553800 & -1.788600 \\ \mathrm{C} & 0.351800 & 1.857800 & -4.124100 \\ \mathrm{C} & 3.661300 & -0.059600 & 2.075600 \\ \mathrm{O} & 2.494600 & 0.608800 & 1.915200 \\ \mathrm{O} & 4.103000 & -0.308100 & 3.175100 \\ \mathrm{C} & 1.869700 & 1.054500 & 3.130400 \\ \mathrm{H} & 4.173500 & -1.536700 & -1.019600 \\ \mathrm{C} & 2.403600 & -0.373000 & -0.711600 \\ \mathrm{H} & 7.705700 & -0.435600 & 1.547600 \\ \mathrm{H} & 6.431500 & 0.154200 & 2.616400 \\ \mathrm{H} & 4.776100 & 1.857900 & 1.828200 \\ \mathrm{H} & 7.710900 & -0.085900 & -2.299900 \\ \mathrm{H} & 8.836900 & 0.497700 & -1.060000 \\ \mathrm{H} & 7.769600 & -0.873400 & -0.719700 \\ \mathrm{H} & 7.063700 & 2.224100 & -2.594300 \\ \mathrm{H} & 6.363200 & 3.189000 & -1.269900 \\ \mathrm{H} & 9.615300 & 4.451400 & -0.873200 \\ \mathrm{H} & 7.973600 & 4.873400 & -1.473600 \\ \mathrm{H} & 9.276900 & 6.035500 & 0.957000\end{array}$

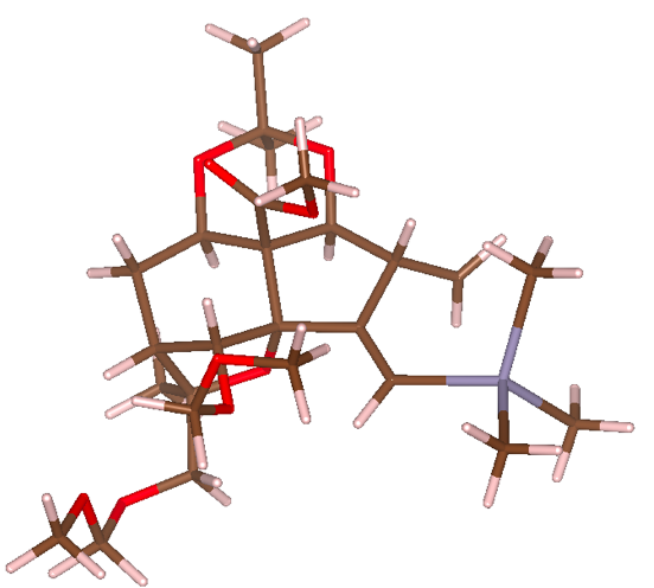




$\begin{array}{rrrr}\mathrm{H} & 7.862300 & 5.738500 & 2.003000 \\ \mathrm{H} & 7.632300 & 6.402600 & 0.362300 \\ \mathrm{H} & 3.249400 & 3.021600 & -1.259200 \\ \mathrm{H} & 4.779600 & -4.543800 & 2.668900 \\ \mathrm{H} & 3.523300 & -3.331600 & 3.015200 \\ \mathrm{H} & 3.180600 & -4.671300 & 1.884000 \\ \mathrm{H} & 5.440900 & -3.289400 & -0.845400 \\ \mathrm{H} & 5.924700 & -4.490400 & 0.360800 \\ \mathrm{H} & 4.338300 & -4.616400 & -0.446500 \\ \mathrm{H} & -0.811100 & 0.253600 & -1.048400 \\ \mathrm{H} & -1.777000 & 1.726200 & -0.789400 \\ \mathrm{H} & -0.809100 & 4.804800 & -2.077700 \\ \mathrm{H} & 0.904600 & 5.135900 & -2.409200 \\ \mathrm{H} & 0.519400 & 0.783400 & -4.235700 \\ \mathrm{H} & -0.657900 & 2.100400 & -4.469300 \\ \mathrm{H} & 0.940800 & 1.532800 & 2.817100 \\ \mathrm{H} & 2.519100 & 1.771200 & 3.641500 \\ \mathrm{H} & 1.668100 & 0.209900 & 3.793700 \\ \mathrm{C} & 1.866100 & -0.823700 & -2.024100 \\ \mathrm{H} & 2.452200 & -0.677400 & -2.926500 \\ \mathrm{H} & 0.999400 & -1.474800 & -2.076100 \\ \mathrm{H} & 1.599000 & -0.343200 & 0.028200 \\ \mathrm{H} & -0.411700 & 1.351400 & 0.289900 \\ \mathrm{H} & 0.363200 & 4.843100 & -0.742900 \\ \mathrm{H} & 1.075000 & 2.395100 & -4.744700 \\ \mathrm{O} & 5.058100 & 3.266400 & 0.389900 \\ \mathrm{C} & 5.106100 & 4.195400 & 1.450200 \\ \mathrm{O} & 4.029200 & 4.082700 & 2.341700 \\ \mathrm{H} & 6.008400 & 4.059200 & 2.054600 \\ \mathrm{H} & 5.120500 & 5.178000 & 0.958000 \\ \mathrm{C} & 2.755000 & 4.265200 & 1.730200 \\ \mathrm{H} & 2.028400 & 4.339800 & 2.543100 \\ \mathrm{H} & 2.497900 & 3.420600 & 1.081800 \\ \mathrm{H} & 2.726100 & 5.191600 & 1.135500\end{array}$

Tomanik et al. "Development of an enantioselective synthesis of (-)-euonyminol." J. Org. Chem. 
Compound 74:

$\begin{array}{lrrr}\mathrm{C} & 0.110000 & -2.794800 & -0.956400 \\ \mathrm{C} & 0.532200 & -0.986400 & 0.859400 \\ \mathrm{C} & -0.442100 & -2.105200 & 0.326400 \\ \mathrm{C} & -0.501600 & -3.271400 & 1.332700 \\ \mathrm{C} & -0.661600 & -2.847100 & 2.790100 \\ \mathrm{C} & 0.318900 & -1.679900 & 3.101900 \\ \mathrm{C} & -0.070600 & -0.488900 & 2.196600 \\ \mathrm{O} & 1.774800 & -1.650300 & 1.234800 \\ \mathrm{C} & 1.788000 & -1.923300 & 2.665300 \\ \mathrm{C} & 2.363000 & -3.316500 & 2.936700 \\ \mathrm{C} & 2.762000 & -0.897700 & 3.281500 \\ \mathrm{O} & 2.680300 & -1.008400 & 4.709300 \\ \mathrm{C} & 3.384700 & -0.031500 & 5.391200 \\ \mathrm{O} & 2.729300 & 1.214400 & 5.243700 \\ \mathrm{C} & 3.453700 & 2.287700 & 5.809200 \\ \mathrm{C} & 0.795300 & -0.024700 & -0.294300 \\ \mathrm{C} & 0.399900 & 1.254800 & -0.302100 \\ \mathrm{O} & -1.492000 & -4.194600 & 0.892200 \\ \mathrm{O} & -0.871800 & -3.740700 & -1.377000 \\ \mathrm{C} & -1.224500 & -4.743600 & -0.407900 \\ \mathrm{C} & -2.554500 & -5.307300 & -0.884900 \\ \mathrm{C} & -0.136000 & -5.823600 & -0.331700 \\ \mathrm{H} & 0.267100 & -1.433000 & 4.163300 \\ \mathrm{H} & 0.474000 & -3.753700 & 1.254400 \\ \mathrm{~S} \mathrm{~S} & 0.318100 & 2.563100 & -2.017300 \\ \mathrm{C} & -0.563200 & 1.447600 & -3.676700 \\ \mathrm{C} & -0.994200 & 4.202600 & -1.425300 \\ \mathrm{C} & 2.282400 & 3.326000 & -2.582300 \\ \mathrm{C} & -1.844100 & -1.527500 & 0.087500 \\ \mathrm{O} & -2.004100 & -1.012000 & -1.146500 \\ \mathrm{O} & -2.725600 & -1.485600 & 0.922600 \\ \mathrm{C} & -3.285700 & -0.416200 & -1.399000 \\ \mathrm{H} & 1.030900 & -3.310200 & -0.634000 \\ \mathrm{C} & 0.500200 & -1.830300 & -2.011500 \\ \mathrm{C} & 1.402600 & -0.734800 & -1.512400 \\ \mathrm{H} & -0.466800 & -3.706000 & 3.442100 \\ \mathrm{H} & -1.686700 & -2.515200 & 2.971600 \\ \mathrm{H} & -1.152700 & -0.385000 & 2.144300 \\ \mathrm{H} & 3.299200 & -3.431100 & 2.379800 \\ \mathrm{H} & 2.585200 & -3.402600 & 4.004700 \\ \mathrm{H} & 1.701400 & -4.137400 & 2.658600 \\ \mathrm{H} & 3.784700 & -1.134700 & 2.949000 \\ \mathrm{H} & 2.504600 & 0.108500 & 2.959200 \\ \mathrm{H} & 3.402900 & -0.334300 & 6.449400 \\ \mathrm{H} & 4.425600 & 0.053100 & 5.019100\end{array}$

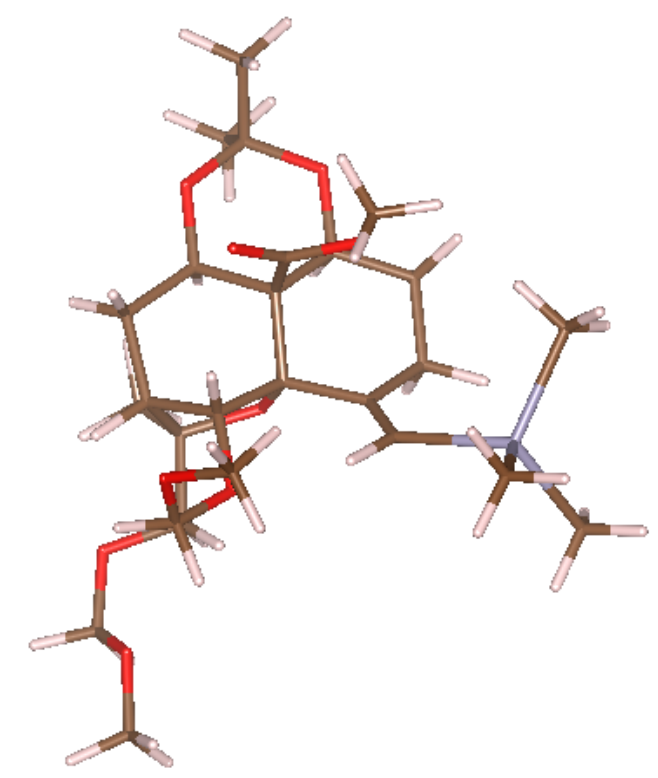

Tomanik et al. "Development of an enantioselective synthesis of (-)-euonyminol." J. Org. Chem. 


$\begin{array}{rrrr}\mathrm{H} & 3.600500 & 2.158700 & 6.894100 \\ \mathrm{H} & 2.868000 & 3.194200 & 5.638900 \\ \mathrm{H} & 4.443000 & 2.408500 & 5.337900 \\ \mathrm{H} & -0.007200 & 1.661900 & 0.617400 \\ \mathrm{H} & -2.899200 & -6.085400 & -0.198300 \\ \mathrm{H} & -3.291700 & -4.500900 & -0.908100 \\ \mathrm{H} & -2.447200 & -5.728400 & -1.888300 \\ \mathrm{H} & 0.827700 & -5.422300 & -0.006800 \\ \mathrm{H} & -0.438800 & -6.599000 & 0.377900 \\ \mathrm{H} & 0.002700 & -6.272600 & -1.319400 \\ \mathrm{H} & -1.035400 & 0.549000 & -3.270900 \\ \mathrm{H} & 0.206400 & 1.145700 & -4.393600 \\ \mathrm{H} & -0.572900 & 4.734300 & -0.566400 \\ \mathrm{H} & -1.976800 & 3.810900 & -1.143400 \\ \mathrm{H} & 2.729600 & 3.874100 & -1.748000 \\ \mathrm{H} & 2.942800 & 2.494800 & -2.847400 \\ \mathrm{H} & -3.242000 & -0.056100 & -2.427100 \\ \mathrm{H} & -3.464100 & 0.412200 & -0.707100 \\ \mathrm{H} & -4.082100 & -1.155800 & -1.279700 \\ \mathrm{H} & 2.366000 & -1.162500 & -1.192000 \\ \mathrm{H} & 1.619400 & -0.012900 & -2.304600 \\ \mathrm{H} & -0.108400 & -1.710300 & -2.898700 \\ \mathrm{H} & -1.314500 & 2.046500 & -4.200100 \\ \mathrm{H} & -1.126000 & 4.914800 & -2.245400 \\ \mathrm{H} & 2.200200 & 3.997800 & -3.442300 \\ \mathrm{O} & 0.483200 & 0.768300 & 2.583100 \\ \mathrm{C} & -0.044200 & 1.309600 & 3.779800 \\ \mathrm{O} & -1.438300 & 1.442100 & 3.773900 \\ \mathrm{H} & 0.196900 & 0.687000 & 4.645300 \\ \mathrm{H} & 0.459600 & 2.280700 & 3.876800 \\ \mathrm{C} & -1.938000 & 2.326000 & 2.781100 \\ \mathrm{H} & -2.988200 & 2.508900 & 3.021700 \\ \mathrm{H} & -1.874300 & 1.890600 & 1.775000 \\ \mathrm{H} & -1.393500 & 3.283300 & 2.783000\end{array}$

Tomanik et al. "Development of an enantioselective synthesis of (-)-euonyminol." J. Org. Chem. 
Compound 75:

C $\quad-1.031600 \quad-2.529000 \quad-2.095700$

C $-0.746300-1.080300 \quad-0.224200$

$\begin{array}{llll}\text { C } & -1.671400 & -2.201500 & -0.755900\end{array}$

$\begin{array}{llll}\text { C } & -1.553300 & -3.456100 & 0.091600\end{array}$

$\begin{array}{llll}\text { C } & -1.745400 & -3.146800 & 1.590200\end{array}$

$\begin{array}{llll}\text { C } & -0.903100 & -1.872400 & 1.974800\end{array}$

$\begin{array}{llll}\text { C } & -1.328000 & -0.630500 & 1.114200\end{array}$

$\begin{array}{llll}\mathrm{O} & 0.536300 & -1.687600 & 0.135900\end{array}$

$\begin{array}{llll}\text { C } & 0.580300 & -2.010700 & 1.553700\end{array}$

$\begin{array}{llll}\text { C } & 1.242200 & -3.379200 & 1.756100\end{array}$

$\begin{array}{llll}\text { C } & 1.519700 & -0.975300 & 2.218300\end{array}$

$\begin{array}{llll}\text { O } & 1.632900 & -1.243900 & 3.623800\end{array}$

C $\quad 1.353100 \quad-0.191200 \quad 4.472600$

$\begin{array}{llll}\mathrm{O} & -0.051000 & -0.016400 & 4.596300\end{array}$

$\begin{array}{llll}\text { C } & -0.382600 & 1.019200 & 5.499800\end{array}$

$\begin{array}{llll}\mathrm{O} & -0.767800 & 0.607500 & 1.546700\end{array}$

$\begin{array}{llll}\text { C } & -1.666300 & 1.408900 & 2.273300\end{array}$

$\begin{array}{llll}\mathrm{O} & -2.732500 & 1.884800 & 1.496600\end{array}$

$\begin{array}{llll}\text { C } & -2.310700 & 2.685600 & 0.396600\end{array}$

$\begin{array}{llll}\text { C } & -0.430100 & -0.205400 & -1.457400\end{array}$

$\begin{array}{llll}\text { C } & 0.739400 & 0.663500 & -1.380500\end{array}$

$\begin{array}{llll}\mathrm{O} & -2.437200 & -4.399100 & -0.533200\end{array}$

$\begin{array}{llll}\mathrm{O} & -1.812100 & -3.528600 & -2.747400\end{array}$

$\mathrm{H} \quad-1.414200 \quad-3.997000 \quad 2.196300$

$\mathrm{H} \quad-2.795700 \quad-2.972100 \quad 1.820800$

$\mathrm{H} \quad-0.999900 \quad-1.668900 \quad 3.043000$

$\mathrm{H} \quad-2.406600 \quad-0.516700 \quad 1.067000$

$\mathrm{H} \quad-0.534100 \quad-3.818900 \quad-0.028500$

$\mathrm{H} \quad 1.443100 \quad 0.411700 \quad-0.592000$

$\begin{array}{llll}\mathrm{H} & -2.122800 & 0.850600 & 3.097900\end{array}$

$\begin{array}{llll}\mathrm{H} & -1.053700 & 2.233300 & 2.666500\end{array}$

$\mathrm{H} \quad-3.216500 \quad 3.022700 \quad-0.113000$

$\begin{array}{llll}\mathrm{H} & -1.683700 & 2.118300 & -0.299700\end{array}$

$\begin{array}{llll}\mathrm{H} & -1.743800 & 3.565400 & 0.740800\end{array}$

$\begin{array}{llll}\mathrm{H} & -1.473000 & 1.074900 & 5.541300\end{array}$

$\begin{array}{llll}\mathrm{H} & 0.001600 & 0.817300 & 6.512900\end{array}$

$\begin{array}{llll}\mathrm{H} & 0.010000 & 1.995000 & 5.168900\end{array}$

$\begin{array}{llll}\mathrm{H} & 1.806800 & 0.754900 & 4.120100\end{array}$

$\mathrm{H} \quad 1.783000 \quad-0.453300 \quad 5.451900$

$\begin{array}{llll}\mathrm{H} & 2.514800 & -1.088900 & 1.768700\end{array}$

$\begin{array}{llll}\mathrm{H} & 1.159100 & 0.034300 & 2.040700\end{array}$

$\mathrm{H} \quad 1.430100 \quad-3.528200 \quad 2.823100$

$\mathrm{H} \quad 2.205200 \quad-3.386000 \quad 1.234300$

$\begin{array}{llll}\mathrm{H} & 0.659300 & -4.223400 & 1.389100\end{array}$

$\begin{array}{llll}\text { C } & -3.191100 & -1.903800 & -0.940200\end{array}$

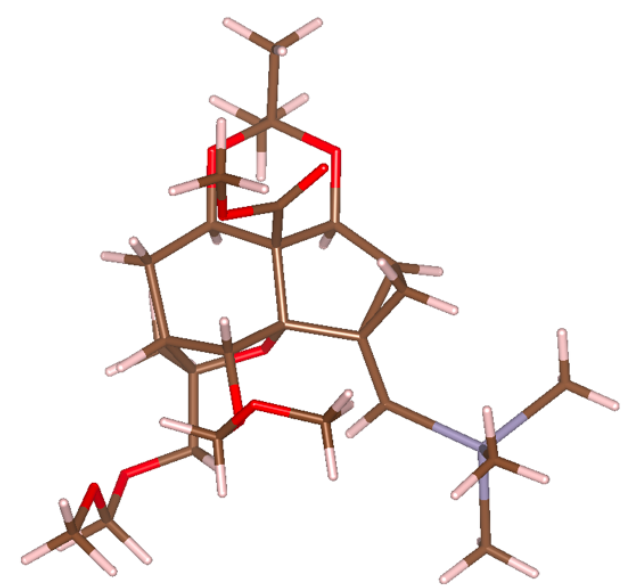

Tomanik et al. "Development of an enantioselective synthesis of (-)-euonyminol." J. Org. Chem. 


$\begin{array}{lrrr}\mathrm{O} & -3.856500 & -1.788800 & 0.234000 \\ \mathrm{O} & -3.778200 & -1.820200 & -1.995100 \\ \mathrm{C} & -5.277000 & -1.604100 & 0.118000 \\ \mathrm{H} & -5.500700 & -0.694800 & -0.445800 \\ \mathrm{H} & -5.641800 & -1.523100 & 1.142300 \\ \mathrm{H} & -5.728400 & -2.460300 & -0.390000 \\ \mathrm{H} & -0.039900 & -2.950000 & -1.880200 \\ \mathrm{C} & -0.718100 & -1.171800 & -2.719700 \\ \mathrm{C} & -1.534500 & 0.053800 & -2.466700 \\ \mathrm{C} & -3.324700 & -5.315100 & -2.515400 \\ \mathrm{C} & -0.869100 & -5.636600 & -1.950900 \\ \mathrm{Sn} & 1.198200 & 2.340700 & -2.630100 \\ \mathrm{C} & -0.344200 & 3.877300 & -2.412000 \\ \mathrm{C} & 3.128000 & 3.124700 & -1.989600 \\ \mathrm{C} & 1.276500 & 1.743000 & -4.731400 \\ \mathrm{H} & -1.329300 & 3.457200 & -2.637100 \\ \mathrm{H} & -0.155800 & 4.712700 & -3.093400 \\ \mathrm{H} & 3.400700 & 4.005500 & -2.578600 \\ \mathrm{H} & 3.905200 & 2.365100 & -2.116300 \\ \mathrm{H} & 0.323300 & 1.300000 & -5.036600 \\ \mathrm{H} & 1.478300 & 2.608900 & -5.369600 \\ \mathrm{H} & 2.068100 & 1.003200 & -4.882800 \\ \mathrm{H} & 3.086900 & 3.409600 & -0.933900 \\ \mathrm{H} & -0.353500 & 4.255400 & -1.385400 \\ \mathrm{H} & 0.000200 & -1.144500 & -3.533500 \\ \mathrm{H} & -1.331100 & 0.882700 & -3.140600 \\ \mathrm{H} & -2.577900 & -0.005500 & -2.197300 \\ \mathrm{C} & -2.075000 & -4.689800 & -1.917800 \\ \mathrm{H} & -3.143100 & -5.582900 & -3.559800 \\ \mathrm{H} & -3.605200 & -6.210300 & -1.953000 \\ \mathrm{H} & -4.135100 & -4.583900 & -2.472300 \\ \mathrm{H} & 0.035700 & -5.171500 & -1.550100 \\ \mathrm{H} & -1.085400 & -6.531400 & -1.359900 \\ \mathrm{H} & -0.666100 & -5.927000 & -2.985600\end{array}$

Tomanik et al. "Development of an enantioselective synthesis of (-)-euonyminol." J. Org. Chem. 
Compound 76:

$\begin{array}{llll}\text { C } & -0.509000 & -2.404200 & -1.972700\end{array}$

$\begin{array}{llll}\text { C } & -0.083100 & -1.171800 & 0.047900\end{array}$

$\begin{array}{llll}\text { C } & -1.200100 & -1.927000 & -0.709200\end{array}$

$\begin{array}{llll}\text { C } & -1.697600 & -3.154400 & 0.014600\end{array}$

$\begin{array}{llll}\text { C } & -2.041000 & -2.848900 & 1.482500\end{array}$

$\begin{array}{llll}\text { C } & -0.908800 & -1.948700 & 2.097900\end{array}$

$\begin{array}{llll}\text { C } & -0.777000 & -0.608400 & 1.297100\end{array}$

$\begin{array}{llll}\mathrm{O} & 0.872500 & -2.135400 & 0.566000\end{array}$

$\begin{array}{llll}\text { C } & 0.518600 & -2.529000 & 1.920100\end{array}$

$\begin{array}{llll}\text { C } & 0.680500 & -4.042800 & 2.092600\end{array}$

$\begin{array}{llll}\text { C } & 1.550700 & -1.858400 & 2.850700\end{array}$

$\begin{array}{llll}\mathrm{O} & 1.153500 & -2.110700 & 4.206300\end{array}$

C $\quad 1.820300 \quad-1.338800 \quad 5.141300$

$\begin{array}{llll}\mathrm{O} & 1.362000 & -0.001100 & 5.062700\end{array}$

$\begin{array}{llll}\text { C } & 2.089200 & 0.880100 & 5.894400\end{array}$

$\begin{array}{llll}\mathrm{O} & -0.009200 & 0.408400 & 1.931800\end{array}$

$\begin{array}{llll}\text { C } & -0.677300 & 1.071900 & 2.989500\end{array}$

$\begin{array}{llll}\mathrm{O} & -1.892100 & 1.655000 & 2.604700\end{array}$

$\begin{array}{llll}\text { C } & -1.756700 & 2.656200 & 1.603100\end{array}$

$\begin{array}{llll}\text { C } & 0.541000 & -0.342800 & -1.124300\end{array}$

$\begin{array}{llll}\text { C } & 0.496900 & 1.122200 & -1.050300\end{array}$

$\begin{array}{llll}\mathrm{O} & -2.720900 & -3.712600 & -0.823300\end{array}$

$\begin{array}{llll}\mathrm{O} & -1.512200 & -2.964000 & -2.815800\end{array}$

$\mathrm{H} \quad-2.127700 \quad-3.777000 \quad 2.058100$

$\mathrm{H} \quad-2.994400 \quad-2.324700 \quad 1.556900$

H $\quad-1.126500 \quad-1.774400 \quad 3.153500$

$\mathrm{H} \quad-1.760100 \quad-0.197200 \quad 1.076400$

$\mathrm{H} \quad-0.858500 \quad-3.852300 \quad 0.030100$

$\begin{array}{llll}\mathrm{H} & 0.738200 & 1.523600 & -0.067600\end{array}$

$\begin{array}{llll}\mathrm{H} & -0.915500 & 0.387100 & 3.808000\end{array}$

$\begin{array}{llll}\mathrm{H} & 0.045800 & 1.822500 & 3.335300\end{array}$

$\mathrm{H} \quad-2.741100 \quad 3.115300 \quad 1.481700$

$\mathrm{H} \quad-1.433600 \quad 2.230100 \quad 0.646200$

$\begin{array}{llll}\mathrm{H} & -1.032600 & 3.429300 & 1.905200\end{array}$

$\begin{array}{llll}\mathrm{H} & 1.662000 & 1.876400 & 5.755700\end{array}$

$\begin{array}{llll}\mathrm{H} & 2.006700 & 0.604000 & 6.958400\end{array}$

$\mathrm{H} \quad 3.157900 \quad 0.909800 \quad 5.625800$

$\begin{array}{llll}\mathrm{H} & 2.916800 & -1.362800 & 4.982300\end{array}$

$\mathrm{H} \quad 1.589400 \quad-1.766500 \quad 6.128900$

$\begin{array}{llll}\mathrm{H} & 2.539400 & -2.305400 & 2.665200\end{array}$

$\mathrm{H} \quad 1.596900 \quad-0.789900 \quad 2.656800$

$\mathrm{H} \quad 0.612100 \quad-4.287800 \quad 3.156700$

$\mathrm{H} \quad 1.674300 \quad-4.334700 \quad 1.736500$

$\begin{array}{llll}\mathrm{H} & -0.056200 & -4.639600 & 1.555000\end{array}$

C $-2.336800-0.959700 \quad-1.134300$ 


$\begin{array}{lrrr}\mathrm{O} & -3.397700 & -0.991300 & -0.300500 \\ \mathrm{O} & -2.306500 & -0.217100 & -2.092700 \\ \mathrm{C} & -4.469300 & -0.091600 & -0.625800 \\ \mathrm{H} & -4.129200 & 0.946200 & -0.564600 \\ \mathrm{H} & -5.244600 & -0.285900 & 0.115600 \\ \mathrm{H} & -4.838900 & -0.287100 & -1.635800 \\ \mathrm{H} & 0.212400 & -3.186000 & -1.700800 \\ \mathrm{C} & 0.279800 & -1.161800 & -2.414100 \\ \mathrm{H} & -0.013100 & -0.660400 & -3.326600 \\ \mathrm{C} & 1.677200 & -1.052100 & -1.891000 \\ \mathrm{H} & 2.143100 & -1.910900 & -1.416300 \\ \mathrm{H} & 2.364100 & -0.390900 & -2.413300 \\ \mathrm{Sn} & -0.126600 & 2.525100 & -2.552800 \\ \mathrm{C} & -0.007600 & 1.720900 & -4.576000 \\ \mathrm{C} & -2.187500 & 3.152000 & -2.185100 \\ \mathrm{C} & 1.173400 & 4.273900 & -2.368400 \\ \mathrm{H} & 0.938600 & 1.196000 & -4.737100 \\ \mathrm{H} & -0.083000 & 2.534700 & -5.304000 \\ \mathrm{H} & -2.519200 & 3.847000 & -2.963000 \\ \mathrm{H} & -2.279500 & 3.644800 & -1.212600 \\ \mathrm{H} & 2.202900 & 4.015900 & -2.634100 \\ \mathrm{H} & 0.831400 & 5.076400 & -3.029700 \\ \mathrm{H} & 1.164100 & 4.645400 & -1.338600 \\ \mathrm{H} & -2.814700 & 2.258100 & -2.202700 \\ \mathrm{H} & -0.834600 & 1.024400 & -4.740000 \\ \mathrm{C} & -2.268400 & -4.023300 & -2.166700 \\ \mathrm{C} & -1.455900 & -5.324100 & -2.164600 \\ \mathrm{C} & -3.543000 & -4.142300 & -2.986600 \\ \mathrm{H} & -0.536400 & -5.240800 & -1.579400 \\ \mathrm{H} & -1.180300 & -5.583900 & -3.190700 \\ \mathrm{H} & -2.058800 & -6.130300 & -1.736800 \\ \mathrm{H} & -3.299200 & -4.380900 & -4.025300 \\ \mathrm{H} & -4.074700 & -3.188000 & -2.955300 \\ \mathrm{H} & -4.184300 & -4.925400 & -2.572900\end{array}$

Tomanik et al. "Development of an enantioselective synthesis of (-)-euonyminol." J. Org. Chem. 
Transition state TS-1:

$\begin{array}{llll}\text { C } & 0.030700 & -2.778600 & -0.999200\end{array}$

$\begin{array}{llll}\text { C } & 0.487500 & -0.947800 & 0.873200\end{array}$

$\begin{array}{llll}\text { C } & -0.449700 & -2.104500 & 0.330400\end{array}$

$\begin{array}{llll}\text { C } & -0.383800 & -3.300700 & 1.319500\end{array}$

$\begin{array}{llll}\text { C } & -0.469400 & -2.945300 & 2.801900\end{array}$

$\begin{array}{llll}\text { C } & 0.446500 & -1.732100 & 3.108300\end{array}$

$\begin{array}{llll}\text { C } & -0.092500 & -0.557200 & 2.270300\end{array}$

$\begin{array}{llll}\mathrm{O} & 1.783500 & -1.548400 & 1.158400\end{array}$

$\begin{array}{llll}\text { C } & 1.898700 & -1.856500 & 2.577000\end{array}$

$\begin{array}{llll}\text { C } & 2.582500 & -3.213200 & 2.762400\end{array}$

$\begin{array}{llll}\text { C } & 2.826200 & -0.779800 & 3.174900\end{array}$

$\begin{array}{llll}\text { O } & 2.912900 & -1.002500 & 4.589800\end{array}$

$\begin{array}{llll}\text { C } & 3.381900 & 0.084400 & 5.305800\end{array}$

$\begin{array}{llll}\mathrm{O} & 2.379300 & 1.083500 & 5.357200\end{array}$

$\begin{array}{llll}\text { C } & 2.825700 & 2.282000 & 5.957800\end{array}$

$\begin{array}{llll}\text { C } & 0.661100 & 0.149700 & -0.121100\end{array}$

$\begin{array}{llll}\text { C } & 0.458900 & 1.447400 & -0.160000\end{array}$

$\begin{array}{llll}\mathrm{O} & -1.354900 & -4.270500 & 0.952400\end{array}$

$\begin{array}{llll}\mathrm{O} & -0.957400 & -3.754100 & -1.338400\end{array}$

$\begin{array}{llll}\text { C } & -1.183300 & -4.789100 & -0.370700\end{array}$

$\begin{array}{llll}\text { C } & -2.526500 & -5.399500 & -0.741900\end{array}$

$\begin{array}{llll}\text { C } & -0.048300 & -5.822400 & -0.411600\end{array}$

$\mathrm{H} \quad 0.448000 \quad-1.518500 \quad 4.179500$

$\mathrm{H} \quad 0.608500 \quad-3.727000 \quad 1.159600$

$\begin{array}{llll}\text { Sn } & 0.520300 & 2.627400 & -1.977500\end{array}$

$\begin{array}{llll}\text { C } & -0.826800 & 1.664300 & -3.401500\end{array}$

$\begin{array}{llll}\text { C } & -0.200900 & 4.624100 & -1.468200\end{array}$

$\begin{array}{llll}\text { C } & 2.544500 & 2.747900 & -2.783200\end{array}$

$\begin{array}{lrrr}\text { C } & -1.884600 & -1.587700 & 0.190500\end{array}$

$\begin{array}{llll}\mathrm{O} & -2.072600 & -0.899200 & -0.953300\end{array}$

$\begin{array}{llll}\mathrm{O} & -2.760300 & -1.715500 & 1.021700\end{array}$

C $-3.377500-0.323500 \quad-1.117600$

$\begin{array}{llll}\mathrm{H} & 0.989500 & -3.258100 & -0.758100\end{array}$

$\begin{array}{llll}\text { C } & 0.268800 & -1.839600 & -2.131400\end{array}$

$\begin{array}{llll}\text { C } & 1.338700 & -1.005200 & -2.034200\end{array}$

$\mathrm{H} \quad-0.179900 \quad-3.821100 \quad 3.393100$

$\mathrm{H} \quad-1.502000 \quad-2.696400 \quad 3.060600$

$\mathrm{H} \quad-1.181300 \quad-0.552000 \quad 2.264000$

$\mathrm{H} \quad 3.509000 \quad-3.229500 \quad 2.178400$

$\begin{array}{llll}\mathrm{H} & 2.839500 & -3.340700 & 3.818000\end{array}$

$\mathrm{H} \quad 1.972700 \quad-4.062300 \quad 2.452000$

$\mathrm{H} \quad 3.824000 \quad-0.879800 \quad 2.721800$

$\begin{array}{llll}\mathrm{H} & 2.431800 & 0.212500 & 2.965700\end{array}$

$\mathrm{H} \quad 3.613900 \quad-0.278600 \quad 6.318700$

$\begin{array}{llll}\mathrm{H} & 4.300400 & 0.506900 & 4.852300\end{array}$

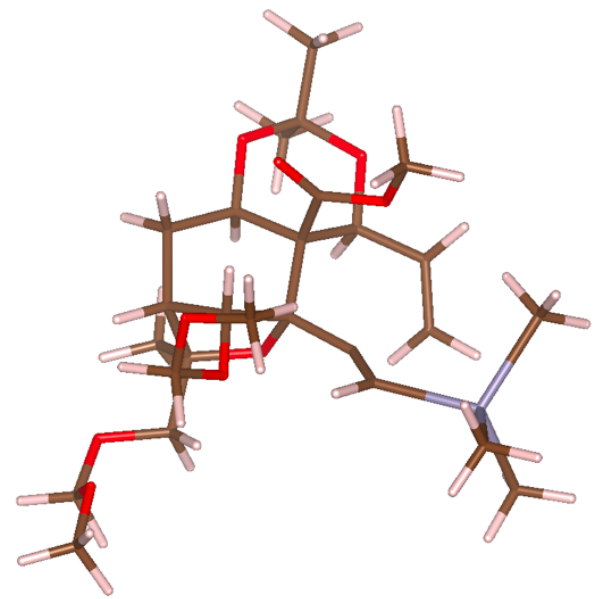




$\begin{array}{rrrr}\mathrm{H} & 3.136900 & 2.127600 & 7.003800 \\ \mathrm{H} & 1.985200 & 2.980300 & 5.940900 \\ \mathrm{H} & 3.670400 & 2.727300 & 5.406800 \\ \mathrm{H} & 0.174300 & 1.972700 & 0.755300 \\ \mathrm{H} & -2.777300 & -6.203600 & -0.044500 \\ \mathrm{H} & -3.293600 & -4.623600 & -0.680700 \\ \mathrm{H} & -2.492000 & -5.798100 & -1.759600 \\ \mathrm{H} & 0.920900 & -5.386400 & -0.154600 \\ \mathrm{H} & -0.259200 & -6.625400 & 0.300500 \\ \mathrm{H} & 0.027900 & -6.243600 & -1.418200 \\ \mathrm{H} & -1.073500 & 0.675900 & -3.006100 \\ \mathrm{H} & -0.358700 & 1.553400 & -4.384000 \\ \mathrm{H} & 0.461000 & 5.092400 & -0.733300 \\ \mathrm{H} & -1.207500 & 4.563200 & -1.042500 \\ \mathrm{H} & 3.186400 & 3.310000 & -2.098600 \\ \mathrm{H} & 2.963000 & 1.745100 & -2.907500 \\ \mathrm{H} & -3.342600 & 0.207000 & -2.069500 \\ \mathrm{H} & -3.594900 & 0.367200 & -0.297600 \\ \mathrm{H} & -4.141700 & -1.105300 & -1.134600 \\ \mathrm{H} & 2.188000 & -1.256400 & -1.405000 \\ \mathrm{H} & 1.507900 & -0.226300 & -2.770900 \\ \mathrm{H} & -0.534900 & -1.676400 & -2.839500 \\ \mathrm{H} & -1.745900 & 2.247700 & -3.513500 \\ \mathrm{H} & -0.237900 & 5.259500 & -2.358400 \\ \mathrm{H} & 2.539400 & 3.251300 & -3.755000 \\ \mathrm{O} & 0.354600 & 0.732000 & 2.676400 \\ \mathrm{C} & -0.291000 & 1.235200 & 3.830400 \\ \mathrm{O} & -1.684100 & 1.312400 & 3.707500 \\ \mathrm{H} & -0.099800 & 0.608400 & 4.705700 \\ \mathrm{H} & 0.161800 & 2.225300 & 3.978500 \\ \mathrm{C} & -2.130800 & 2.162300 & 2.660700 \\ \mathrm{H} & -3.210200 & 2.275400 & 2.787400 \\ \mathrm{H} & -1.926500 & 1.733100 & 1.671100 \\ \mathrm{H} & -1.653200 & 3.153600 & 2.716200\end{array}$

Tomanik et al. "Development of an enantioselective synthesis of (-)-euonyminol." J. Org. Chem. 
Transition state TS-2:

$\begin{array}{llll}\text { C } & 3.508600 & -1.329100 & -0.185300\end{array}$

$\begin{array}{llll}\text { C } & 4.495200 & 0.941100 & -0.149800\end{array}$

$\begin{array}{llll}\text { C } & 4.295100 & -0.351900 & 0.680300\end{array}$

$\begin{array}{llll}\text { C } & 5.642400 & -1.020200 & 0.945700\end{array}$

$\begin{array}{llll}\text { C } & 6.645400 & -0.030800 & 1.560200\end{array}$

$\begin{array}{llll}\text { C } & 6.627300 & 1.314400 & 0.758400\end{array}$

$\begin{array}{llll}\text { C } & 5.192600 & 1.916500 & 0.823100\end{array}$

$\begin{array}{llll}\text { O } & 5.469200 & 0.704700 & -1.204500\end{array}$

$\begin{array}{llll}\text { C } & 6.798900 & 1.116600 & -0.767500\end{array}$

$\begin{array}{llll}\text { C } & 7.837200 & 0.085600 & -1.220100\end{array}$

$\begin{array}{llll}\text { C } & 7.111000 & 2.432600 & -1.515000\end{array}$

$\begin{array}{llll}\text { O } & 8.438500 & 2.868900 & -1.184500\end{array}$

$\begin{array}{llll}\text { C } & 8.567200 & 4.192200 & -0.813300\end{array}$

$\begin{array}{llll}\text { O } & 8.110800 & 4.371800 & 0.519600\end{array}$

$\begin{array}{llll}\text { C } & 8.253200 & 5.708400 & 0.958500\end{array}$

$\begin{array}{llll}\text { C } & 3.177000 & 1.237100 & -0.795200\end{array}$

$\begin{array}{llll}\text { C } & 2.635300 & 2.324400 & -1.299300\end{array}$

$\begin{array}{llll}\text { O } & 5.425800 & -2.203900 & 1.705200\end{array}$

$\begin{array}{llll}\text { O } & 3.286200 & -2.495700 & 0.612900\end{array}$

$\begin{array}{llll}\text { C } & 4.493700 & -3.122000 & 1.109600\end{array}$

$\begin{array}{llll}\text { C } & 4.029500 & -4.009600 & 2.253600\end{array}$

$\begin{array}{llll}\text { C } & 5.168700 & -3.919600 & -0.014400\end{array}$

$\begin{array}{llll}\mathrm{H} & 7.374800 & 2.006300 & 1.153700\end{array}$

$\begin{array}{llll}\mathrm{H} & 6.022500 & -1.308500 & -0.036600\end{array}$

$\begin{array}{llll}\text { Sn } & 0.599700 & 2.505500 & -2.011400\end{array}$

$\begin{array}{lrrr}\text { C } & -0.692900 & 1.381100 & -0.658800\end{array}$

$\begin{array}{llll}\text { C } & 0.125400 & 4.631200 & -1.855900\end{array}$

$\begin{array}{llll}\text { C } & 0.389000 & 1.839300 & -4.076100\end{array}$

$\begin{array}{llll}\text { C } & 3.574600 & -0.049700 & 2.011600\end{array}$

$\begin{array}{llll}\mathrm{O} & 2.399300 & 0.597200 & 1.825000\end{array}$

$\begin{array}{llll}\mathrm{O} & 4.003600 & -0.279200 & 3.120300\end{array}$

$\begin{array}{llll}\text { C } & 1.742300 & 1.028300 & 3.028800\end{array}$

$\mathrm{H} \quad 4.143800 \quad-1.568600 \quad-1.050200$

$\begin{array}{llll}\text { C } & 2.240600 & -0.713400 & -0.740000\end{array}$

$\begin{array}{llll}\mathrm{H} & 7.652000 & -0.461800 & 1.551100\end{array}$

$\begin{array}{llll}\mathrm{H} & 6.379000 & 0.158300 & 2.603000\end{array}$

$\mathrm{H} \quad 4.779700 \quad 1.842100 \quad 1.829300$

$\mathrm{H} \quad 7.722800 \quad-0.085300 \quad-2.295900$

$\begin{array}{lllll}\mathrm{H} & 8.839000 & 0.485700 & -1.041200\end{array}$

H $\quad 7.755300 \quad-0.877600 \quad-0.716900$

$\begin{array}{llll}\mathrm{H} & 7.067600 & 2.229600 & -2.592900\end{array}$

$\begin{array}{llll}\mathrm{H} & 6.375800 & 3.193700 & -1.262900\end{array}$

$\begin{array}{llll}\mathrm{H} & 9.639000 & 4.435200 & -0.877900\end{array}$

$\begin{array}{llll}\mathrm{H} & 8.002400 & 4.867700 & -1.484500\end{array}$

$\begin{array}{llll}\mathrm{H} & 9.306000 & 6.033800 & 0.944000\end{array}$

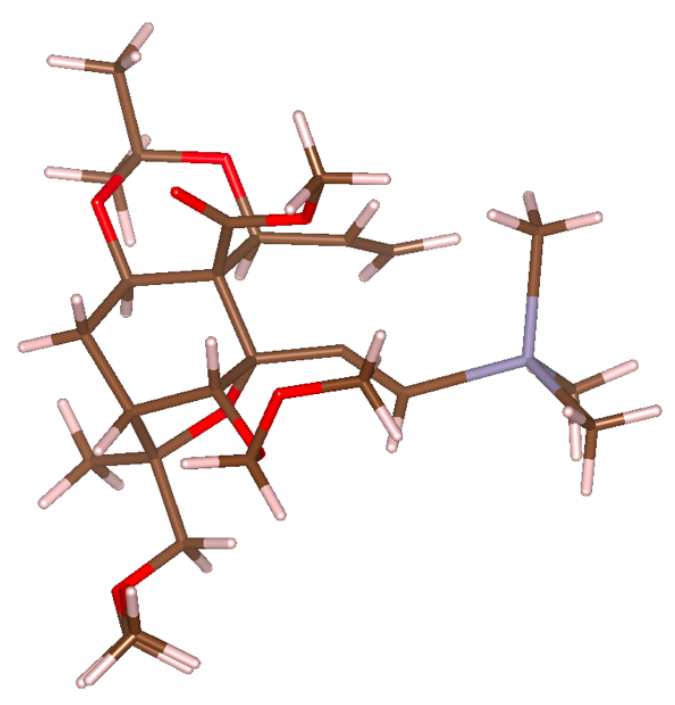

Tomanik et al. "Development of an enantioselective synthesis of (-)-euonyminol." J. Org. Chem. 


$\begin{array}{rrrr}\mathrm{H} & 7.885700 & 5.752100 & 1.986500 \\ \mathrm{H} & 7.666000 & 6.408500 & 0.341200 \\ \mathrm{H} & 3.225200 & 3.244600 & -1.252600 \\ \mathrm{H} & 4.883600 & -4.537000 & 2.687600 \\ \mathrm{H} & 3.573300 & -3.378600 & 3.020000 \\ \mathrm{H} & 3.296600 & -4.737000 & 1.893500 \\ \mathrm{H} & 5.505700 & -3.278100 & -0.833300 \\ \mathrm{H} & 6.040900 & -4.445500 & 0.384800 \\ \mathrm{H} & 4.462900 & -4.648400 & -0.423200 \\ \mathrm{H} & -0.785000 & 0.339300 & -0.977200 \\ \mathrm{H} & -1.691400 & 1.826700 & -0.619000 \\ \mathrm{H} & -0.910900 & 4.820900 & -2.152000 \\ \mathrm{H} & 0.784900 & 5.216700 & -2.504000 \\ \mathrm{H} & 0.822700 & 0.841900 & -4.184100 \\ \mathrm{H} & -0.666600 & 1.804100 & -4.362700 \\ \mathrm{H} & 0.822200 & 1.511600 & 2.697700 \\ \mathrm{H} & 2.378100 & 1.735600 & 3.569100 \\ \mathrm{H} & 1.521900 & 0.174900 & 3.674800 \\ \mathrm{C} & 1.797300 & -1.096200 & -2.002400 \\ \mathrm{H} & 2.473400 & -1.547000 & -2.724200 \\ \mathrm{H} & 0.789600 & -0.879900 & -2.341800 \\ \mathrm{H} & 1.490700 & -0.441600 & -0.004900 \\ \mathrm{H} & -0.253600 & 1.399900 & 0.343100 \\ \mathrm{H} & 0.260000 & 4.971600 & -0.824200 \\ \mathrm{H} & 0.913800 & 2.526400 & -4.746200 \\ \mathrm{O} & 5.070100 & 3.272400 & 0.412200 \\ \mathrm{C} & 5.151000 & 4.188700 & 1.482800 \\ \mathrm{O} & 4.093600 & 4.076700 & 2.396100 \\ \mathrm{H} & 6.065100 & 4.036000 & 2.064600 \\ \mathrm{H} & 5.165400 & 5.176500 & 1.000900 \\ \mathrm{C} & 2.808700 & 4.292800 & 1.819800 \\ \mathrm{H} & 2.102700 & 4.359800 & 2.651300 \\ \mathrm{H} & 2.523100 & 3.468100 & 1.158000 \\ \mathrm{H} & 2.780800 & 5.233600 & 1.247700\end{array}$

Tomanik et al. "Development of an enantioselective synthesis of (-)-euonyminol." J. Org. Chem. 
Transition state TS-3:

$\begin{array}{llll}\text { C } & 3.478900 & -1.302100 & -0.360400\end{array}$

$\begin{array}{llll}\text { C } & 4.420800 & 0.982000 & -0.151900\end{array}$

$\begin{array}{llll}\text { C } & 4.236400 & -0.366200 & 0.582500\end{array}$

$\begin{array}{llll}\text { C } & 5.591300 & -1.032500 & 0.815700\end{array}$

$\begin{array}{llll}\text { C } & 6.569300 & -0.072100 & 1.509400\end{array}$

$\begin{array}{llll}\text { C } & 6.541500 & 1.322700 & 0.797400\end{array}$

$\begin{array}{llll}\text { C } & 5.098800 & 1.898400 & 0.890400\end{array}$

$\begin{array}{llll}\text { O } & 5.406100 & 0.835900 & -1.214200\end{array}$

$\begin{array}{llll}\text { C } & 6.727700 & 1.231200 & -0.737100\end{array}$

$\begin{array}{llll}\text { C } & 7.783600 & 0.247100 & -1.249800\end{array}$

$\begin{array}{llll}\text { C } & 7.029500 & 2.599200 & -1.389600\end{array}$

$\begin{array}{llll}\text { O } & 8.347700 & 3.029100 & -1.016500\end{array}$

$\begin{array}{llll}\text { C } & 8.454800 & 4.325400 & -0.554100\end{array}$

$\begin{array}{llll}\text { O } & 7.984100 & 4.406800 & 0.783400\end{array}$

$\begin{array}{llll}\text { C } & 8.101900 & 5.712400 & 1.313600\end{array}$

$\begin{array}{llll}\text { C } & 3.100400 & 1.306000 & -0.784000\end{array}$

$\begin{array}{llll}\text { C } & 2.560900 & 2.419200 & -1.231400\end{array}$

$\begin{array}{llll}\mathrm{O} & 5.391700 & -2.269100 & 1.488400\end{array}$

$\begin{array}{llll}\mathrm{O} & 3.258600 & -2.513100 & 0.364700\end{array}$

$\begin{array}{llll}\text { C } & 4.467000 & -3.156800 & 0.838000\end{array}$

$\begin{array}{llll}\text { C } & 3.998700 & -4.106800 & 1.928900\end{array}$

$\begin{array}{llll}\text { C } & 5.156500 & -3.886900 & -0.322300\end{array}$

$\begin{array}{llll}\mathrm{H} & 7.276300 & 1.996200 & 1.244500\end{array}$

H $\quad 5.985800 \quad-1.244200 \quad-0.180500$

$\begin{array}{llll}\text { Sn } & 0.524700 & 2.640000 & -1.929000\end{array}$

$\begin{array}{lrrr}\text { C } & -0.757000 & 2.453100 & -0.172000\end{array}$

$\begin{array}{llll}\text { C } & 0.351100 & 4.648500 & -2.763800\end{array}$

$\begin{array}{llll}\text { C } & 0.023500 & 1.167300 & -3.456800\end{array}$

$\begin{array}{llll}\text { C } & 3.495300 & -0.163100 & 1.921600\end{array}$

$\begin{array}{llll}\mathrm{O} & 2.388800 & 0.598000 & 1.771400\end{array}$

$\begin{array}{llll}\text { O } & 3.858300 & -0.558500 & 3.007200\end{array}$

$\begin{array}{llll}\text { C } & 1.700800 & 0.937700 & 2.985500\end{array}$

$\mathrm{H} \quad 4.149100 \quad-1.482500 \quad-1.211700$

$\begin{array}{llll}\text { C } & 2.223500 & -0.662100 & -0.940600\end{array}$

$\begin{array}{llll}\mathrm{H} & 7.582300 & -0.487300 & 1.490600\end{array}$

$\begin{array}{llll}\mathrm{H} & 6.282900 & 0.044500 & 2.558300\end{array}$

$\begin{array}{llll}\mathrm{H} & 4.680500 & 1.751500 & 1.886300\end{array}$

$\begin{array}{llll}\mathrm{H} & 7.675700 & 0.143600 & -2.334900\end{array}$

$\begin{array}{llll}\mathrm{H} & 8.778200 & 0.650700 & -1.041200\end{array}$

H $\quad 7.716200 \quad-0.747600 \quad-0.809500$

$\begin{array}{llll}\mathrm{H} & 6.999200 & 2.469800 & -2.479200\end{array}$

$\begin{array}{lllll}\mathrm{H} & 6.281600 & 3.331700 & -1.093800\end{array}$

$\begin{array}{llll}\mathrm{H} & 9.523600 & 4.586500 & -0.591400\end{array}$

$\begin{array}{llll}\mathrm{H} & 7.886500 & 5.037900 & -1.182900\end{array}$

$\begin{array}{llll}\mathrm{H} & 9.149500 & 6.053700 & 1.331700\end{array}$

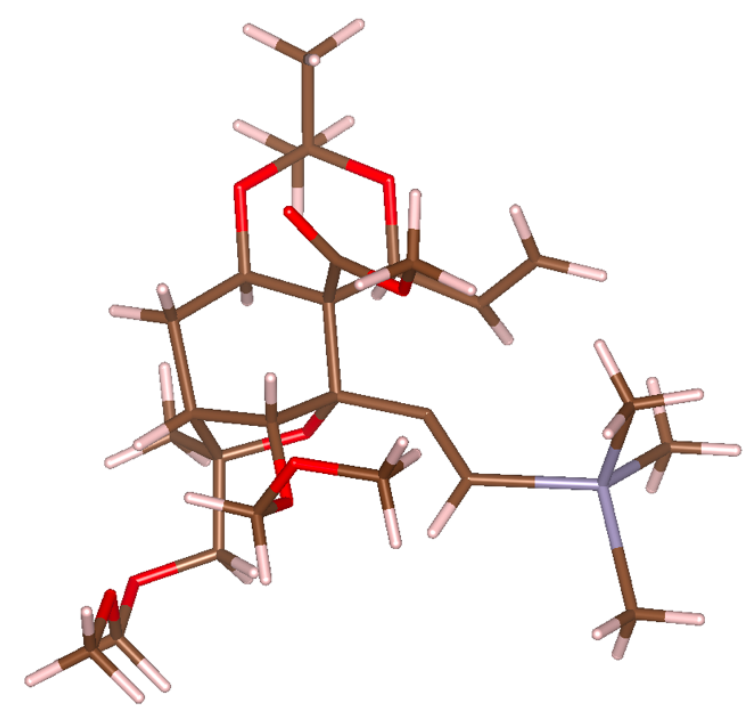

Tomanik et al. "Development of an enantioselective synthesis of (-)-euonyminol." J. Org. Chem. 


$\begin{array}{rrrr}\mathrm{H} & 7.724600 & 5.680300 & 2.338500 \\ \mathrm{H} & 7.509200 & 6.444000 & 0.739700 \\ \mathrm{H} & 3.153300 & 3.334800 & -1.141100 \\ \mathrm{H} & 4.852500 & -4.651300 & 2.341600 \\ \mathrm{H} & 3.532800 & -3.518800 & 2.723000 \\ \mathrm{H} & 3.273800 & -4.818600 & 1.524000 \\ \mathrm{H} & 5.500800 & -3.197600 & -1.098500 \\ \mathrm{H} & 6.026500 & -4.431300 & 0.056200 \\ \mathrm{H} & 4.459000 & -4.594000 & -0.780500 \\ \mathrm{H} & -0.635100 & 3.332400 & 0.468600 \\ \mathrm{H} & -0.452000 & 1.562900 & 0.384600 \\ \mathrm{H} & 0.646600 & 5.394000 & -2.018900 \\ \mathrm{H} & -0.680200 & 4.851900 & -3.068000 \\ \mathrm{H} & -1.009000 & 1.308300 & -3.791300 \\ \mathrm{H} & 0.689900 & 1.288000 & -4.315800 \\ \mathrm{H} & 0.821200 & 1.499000 & 2.668400 \\ \mathrm{H} & 2.344800 & 1.554100 & 3.619500 \\ \mathrm{H} & 1.412200 & 0.036200 & 3.531900 \\ \mathrm{H} & 2.220200 & -0.601400 & -2.027300 \\ \mathrm{C} & 0.981600 & -0.830400 & -0.337200 \\ \mathrm{H} & 0.063300 & -0.604500 & -0.868900 \\ \mathrm{H} & 0.892600 & -1.159500 & 0.690300 \\ \mathrm{H} & -1.811900 & 2.362600 & -0.448200 \\ \mathrm{H} & 0.999900 & 4.753800 & -3.638600 \\ \mathrm{H} & 0.131200 & 0.150700 & -3.070700 \\ \mathrm{O} & 4.960700 & 3.276900 & 0.570100 \\ \mathrm{C} & 5.019900 & 4.120600 & 1.699900 \\ \mathrm{O} & 3.949900 & 3.942500 & 2.587400 \\ \mathrm{H} & 5.926600 & 3.935000 & 2.283900 \\ \mathrm{H} & 5.035100 & 5.138300 & 1.285100 \\ \mathrm{C} & 2.673300 & 4.196400 & 2.007000 \\ \mathrm{H} & 1.953000 & 4.196200 & 2.828800 \\ \mathrm{H} & 2.403500 & 3.423300 & 1.280000 \\ \mathrm{H} & 2.651200 & 5.179100 & 1.509900\end{array}$

Tomanik et al. "Development of an enantioselective synthesis of (-)-euonyminol." J. Org. Chem. 
Transition state TS-4:

C $-0.645600 \quad-2.358300 \quad-1.952400$

$\begin{array}{llll}\text { C } & -0.150900 & -1.136800 & 0.054300\end{array}$

$\begin{array}{llll}\text { C } & -1.283300 & -1.925100 & -0.649200\end{array}$

$\begin{array}{llll}\text { C } & -1.689500 & -3.184300 & 0.076000\end{array}$

$\begin{array}{llll}\text { C } & -1.949900 & -2.923500 & 1.569300\end{array}$

$\begin{array}{llll}\text { C } & -0.812400 & -1.993900 & 2.130400\end{array}$

$\begin{array}{llll}\text { C } & -0.795300 & -0.633800 & 1.354000\end{array}$

$\begin{array}{llll}\mathrm{O} & 0.881700 & -2.062300 & 0.483200\end{array}$

$\begin{array}{llll}\text { C } & 0.623800 & -2.508000 & 1.846200\end{array}$

$\begin{array}{llll}\text { C } & 0.862400 & -4.016600 & 1.963200\end{array}$

$\begin{array}{llll}\text { C } & 1.676800 & -1.813100 & 2.733800\end{array}$

$\begin{array}{llll}\text { O } & 1.426900 & -2.187700 & 4.097000\end{array}$

$\begin{array}{llll}\text { C } & 1.945100 & -1.309100 & 5.031300\end{array}$

$\begin{array}{llll}\mathrm{O} & 1.162300 & -0.128400 & 5.052500\end{array}$

$\begin{array}{llll}\mathrm{C} & 1.703100 & 0.876400 & 5.886300\end{array}$

$\begin{array}{llll}\mathrm{O} & -0.056900 & 0.416100 & 1.968500\end{array}$

$\begin{array}{llll}\text { C } & -0.763300 & 1.091000 & 2.993500\end{array}$

$\begin{array}{llll}\mathrm{O} & -1.979600 & 1.644000 & 2.570600\end{array}$

$\begin{array}{lrrr}\text { C } & -1.852000 & 2.616500 & 1.538900\end{array}$

$\begin{array}{llll}\text { C } & 0.296600 & -0.253600 & -1.131900\end{array}$

$\begin{array}{llll}\text { C } & 0.436700 & 1.122300 & -1.034700\end{array}$

$\begin{array}{llll}\mathrm{O} & -2.740400 & -3.762100 & -0.713200\end{array}$

$\begin{array}{llll}\mathrm{O} & -1.670900 & -2.933300 & -2.755900\end{array}$

$\mathrm{H} \quad-1.965900 \quad-3.866600 \quad 2.126400$

$\mathrm{H} \quad-2.915100 \quad-2.436200 \quad 1.715900$

H $\quad-0.960900 \quad-1.848100 \quad 3.202800$

$\mathrm{H} \quad-1.809300 \quad-0.274200 \quad 1.193900$

$\mathrm{H} \quad-0.826700 \quad-3.850600 \quad 0.024700$

$\begin{array}{llll}\mathrm{H} & 0.576800 & 1.505600 & -0.027400\end{array}$

$\begin{array}{llll}\mathrm{H} & -1.009900 & 0.417700 & 3.819300\end{array}$

$\begin{array}{llll}\mathrm{H} & -0.062800 & 1.864100 & 3.337900\end{array}$

$\begin{array}{llll}\mathrm{H} & -2.829100 & 3.095800 & 1.437500\end{array}$

$\begin{array}{llll}\mathrm{H} & -1.570100 & 2.160100 & 0.582800\end{array}$

$\begin{array}{llll}\mathrm{H} & -1.101500 & 3.379700 & 1.798300\end{array}$

$\begin{array}{llll}\mathrm{H} & 1.033300 & 1.737800 & 5.824300\end{array}$

$\begin{array}{llll}\mathrm{H} & 1.764500 & 0.550300 & 6.937300\end{array}$

$\begin{array}{llll}\mathrm{H} & 2.709900 & 1.183300 & 5.558700\end{array}$

$\begin{array}{llll}\mathrm{H} & 2.998600 & -1.048600 & 4.808800\end{array}$

$\begin{array}{llll}\mathrm{H} & 1.897700 & -1.817400 & 6.006400\end{array}$

$\begin{array}{llll}\mathrm{H} & 2.676700 & -2.159300 & 2.433100\end{array}$

$\begin{array}{llll}\mathrm{H} & 1.615800 & -0.734000 & 2.613900\end{array}$

$\mathrm{H} \quad 0.851900 \quad-4.299200 \quad 3.019800$

$\mathrm{H} \quad 1.851800 \quad-4.250800 \quad 1.555800$

$\begin{array}{llll}\mathrm{H} & 0.130400 & -4.628900 & 1.436800\end{array}$

C $\quad-2.472700 \quad-0.992800 \quad-0.994800$

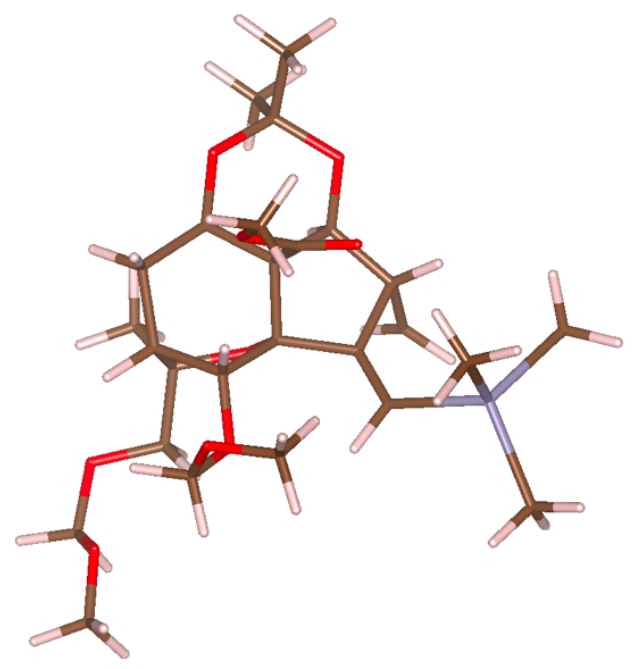

Tomanik et al. "Development of an enantioselective synthesis of (-)-euonyminol." J. Org. Chem. 


$\begin{array}{lrrr}\mathrm{O} & -3.485500 & -1.065900 & -0.105800 \\ \mathrm{O} & -2.510700 & -0.237300 & -1.942600 \\ \mathrm{C} & -4.588200 & -0.179400 & -0.357000 \\ \mathrm{H} & -4.254300 & 0.861900 & -0.330700 \\ \mathrm{H} & -5.304100 & -0.375000 & 0.441400 \\ \mathrm{H} & -5.029400 & -0.387500 & -1.335300 \\ \mathrm{H} & 0.108100 & -3.123000 & -1.729600 \\ \mathrm{C} & 0.113600 & -1.087200 & -2.405200 \\ \mathrm{H} & -0.295400 & -0.582600 & -3.276700 \\ \mathrm{C} & 1.586500 & -1.172800 & -2.269300 \\ \mathrm{H} & 2.052000 & -1.983800 & -1.723700 \\ \mathrm{H} & 2.220500 & -0.430700 & -2.738800 \\ \mathrm{Sn} & 0.087900 & 2.572100 & -2.582200 \\ \mathrm{C} & 0.438900 & 1.772500 & -4.583100 \\ \mathrm{C} & -2.002400 & 3.182000 & -2.399300 \\ \mathrm{C} & 1.390100 & 4.290000 & -2.228400 \\ \mathrm{H} & 1.317700 & 1.121100 & -4.593800 \\ \mathrm{H} & 0.602900 & 2.590800 & -5.291100 \\ \mathrm{H} & -2.309300 & 3.809500 & -3.241800 \\ \mathrm{H} & -2.153500 & 3.742400 & -1.470900 \\ \mathrm{H} & 2.438800 & 4.007700 & -2.361800 \\ \mathrm{H} & 1.154300 & 5.100800 & -2.924700 \\ \mathrm{H} & 1.257800 & 4.660100 & -1.206700 \\ \mathrm{H} & -2.613300 & 2.275800 & -2.374700 \\ \mathrm{H} & -0.430000 & 1.195400 & -4.912500 \\ \mathrm{C} & -2.357100 & -4.029600 & -2.088000 \\ \mathrm{C} & -1.506100 & -5.303300 & -2.161800 \\ \mathrm{C} & -3.672600 & -4.171100 & -2.836000 \\ \mathrm{H} & -0.556800 & -5.202600 & -1.629300 \\ \mathrm{H} & -1.283300 & -5.533100 & -3.207600 \\ \mathrm{H} & -2.057700 & -6.136900 & -1.717600 \\ \mathrm{H} & -3.482200 & -4.379300 & -3.892300 \\ \mathrm{H} & -4.230200 & -3.234900 & -2.751900 \\ \mathrm{H} & -4.264800 & -4.982900 & -2.404800 \\ & & & \\ \end{array}$

Tomanik et al. "Development of an enantioselective synthesis of (-)-euonyminol." J. Org. Chem. 
Transition state TS-5:

C $\quad-1.218900-2.168800 \quad-2.140300$

C $-0.864400 \quad-0.910400-0.157800$

$\begin{array}{llll}\text { C } & -1.787100 & -2.005900 & -0.737000\end{array}$

$\begin{array}{llll}\text { C } & -1.564700 & -3.333000 & -0.034000\end{array}$

$\begin{array}{llll}\text { C } & -1.676800 & -3.179500 & 1.496600\end{array}$

$\begin{array}{llll}\text { C } & -0.850200 & -1.917800 & 1.957000\end{array}$

$\begin{array}{llll}\text { C } & -1.364600 & -0.610000 & 1.252300\end{array}$

$\begin{array}{llll}\mathrm{O} & 0.467900 & -1.500900 & 0.067300\end{array}$

$\begin{array}{llll}\text { C } & 0.607100 & -1.964900 & 1.438500\end{array}$

$\begin{array}{llll}\text { C } & 1.318700 & -3.324100 & 1.461400\end{array}$

$\begin{array}{llll}\text { C } & 1.556200 & -0.970800 & 2.149500\end{array}$

$\begin{array}{llll}\mathrm{O} & 1.777200 & -1.392200 & 3.504600\end{array}$

$\begin{array}{llll}\text { C } & 1.557400 & -0.442300 & 4.481500\end{array}$

$\begin{array}{llll}\mathrm{O} & 0.164200 & -0.287700 & 4.713300\end{array}$

$\begin{array}{llll}\text { C } & -0.105400 & 0.644900 & 5.741200\end{array}$

$\begin{array}{llll}\mathrm{O} & -0.819300 & 0.601900 & 1.773200\end{array}$

$\begin{array}{llll}\text { C } & -1.678700 & 1.257600 & 2.676300\end{array}$

$\begin{array}{llll}\mathrm{O} & -2.863900 & 1.713600 & 2.084300\end{array}$

$\begin{array}{llll}\text { C } & -2.649200 & 2.622300 & 1.007600\end{array}$

$\begin{array}{llll}\text { C } & -0.599100 & 0.025800 & -1.348600\end{array}$

$\begin{array}{llll}\text { C } & 0.227300 & 1.133100 & -1.229000\end{array}$

$\begin{array}{llll}\mathrm{O} & -2.445800 & -4.248100 & -0.702600\end{array}$

$\begin{array}{llll}\mathrm{O} & -1.980400 & -3.130800 & -2.850900\end{array}$

$\mathrm{H} \quad-1.283900-4.071300 \quad 1.996400$

$\mathrm{H} \quad-2.716400 \quad-3.066000 \quad 1.802500$

$\mathrm{H} \quad-0.887200 \quad-1.824600 \quad 3.044500$

$\mathrm{H} \quad-2.445100 \quad-0.530200 \quad 1.287700$

$\mathrm{H} \quad-0.540900 \quad-3.632600 \quad-0.249500$

$\begin{array}{llll}\mathrm{H} & 0.418500 & 1.453400 & -0.207100\end{array}$

$\begin{array}{llll}\mathrm{H} & -1.987900 & 0.588700 & 3.486100\end{array}$

$\begin{array}{llll}\mathrm{H} & -1.079900 & 2.088700 & 3.076700\end{array}$

$\begin{array}{llll}\mathrm{H} & -3.635100 & 2.983300 & 0.704700\end{array}$

$\begin{array}{llll}\mathrm{H} & -2.157200 & 2.135300 & 0.157600\end{array}$

$\begin{array}{llll}\mathrm{H} & -2.034500 & 3.478700 & 1.327700\end{array}$

$\begin{array}{llll}\mathrm{H} & -1.190600 & 0.692500 & 5.858500\end{array}$

$\begin{array}{llll}\mathrm{H} & 0.342200 & 0.337700 & 6.700300\end{array}$

$\begin{array}{llll}\mathrm{H} & 0.268700 & 1.651700 & 5.491500\end{array}$

$\begin{array}{llll}\mathrm{H} & 1.990400 & 0.539500 & 4.208900\end{array}$

$\begin{array}{llll}\mathrm{H} & 2.048500 & -0.810300 & 5.395800\end{array}$

$\begin{array}{llll}\mathrm{H} & 2.518500 & -0.989200 & 1.621900\end{array}$

$\begin{array}{llll}\mathrm{H} & 1.148500 & 0.035700 & 2.115800\end{array}$

$\mathrm{H} \quad 1.572300 \quad-3.576000 \quad 2.494900$

$\begin{array}{llll}\mathrm{H} & 2.249700 & -3.243400 & 0.890100\end{array}$

$\begin{array}{llll}\mathrm{H} & 0.740900 & -4.146100 & 1.039900\end{array}$

$\begin{array}{llll}\text { C } & -3.327100 & -1.767300 & -0.779000\end{array}$

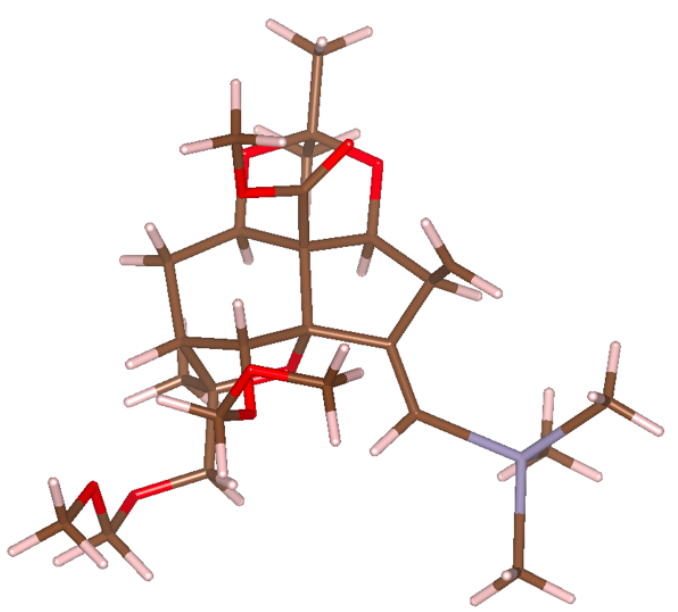

Tomanik et al. "Development of an enantioselective synthesis of (-)-euonyminol." J. Org. Chem. 


$\begin{array}{lrrr}\mathrm{O} & -3.894500 & -1.701500 & 0.448900 \\ \mathrm{O} & -4.013500 & -1.709300 & -1.775000 \\ \mathrm{C} & -5.326700 & -1.579300 & 0.459000 \\ \mathrm{H} & -5.637500 & -0.674500 & -0.069500 \\ \mathrm{H} & -5.603100 & -1.525600 & 1.512300 \\ \mathrm{H} & -5.782900 & -2.448800 & -0.021300 \\ \mathrm{H} & -0.195100 & -2.547700 & -2.013400 \\ \mathrm{C} & -0.987200 & -0.725200 & -2.639700 \\ \mathrm{C} & -2.070600 & 0.280700 & -2.652500 \\ \mathrm{C} & -3.415800 & -4.992500 & -2.718000 \\ \mathrm{C} & -0.919400 & -5.264000 & -2.325300 \\ \mathrm{Sn} & 1.310100 & 2.162200 & -2.774300 \\ \mathrm{C} & -0.023500 & 3.068600 & -4.250600 \\ \mathrm{C} & 2.476400 & 3.716700 & -1.785800 \\ \mathrm{C} & 2.631000 & 0.765200 & -3.811500 \\ \mathrm{H} & -0.498500 & 2.295600 & -4.862700 \\ \mathrm{H} & 0.542400 & 3.733600 & -4.910500 \\ \mathrm{H} & 3.079300 & 4.268000 & -2.513700 \\ \mathrm{H} & 3.146300 & 3.271800 & -1.043700 \\ \mathrm{H} & 2.041500 & 0.004400 & -4.332200 \\ \mathrm{H} & 3.247700 & 1.292600 & -4.546000 \\ \mathrm{H} & 3.285400 & 0.265300 & -3.091700 \\ \mathrm{H} & 1.812100 & 4.422400 & -1.277600 \\ \mathrm{H} & -0.805100 & 3.651600 & -3.754000 \\ \mathrm{H} & -0.264700 & -0.661400 & -3.457800 \\ \mathrm{H} & -1.862800 & 1.234800 & -3.120000 \\ \mathrm{H} & -3.085500 & 0.064100 & -2.369900 \\ \mathrm{C} & -2.156600 & -4.380500 & -2.127900 \\ \mathrm{H} & -3.287300 & -5.141400 & -3.793600 \\ \mathrm{H} & -3.627800 & -5.953200 & -2.240100 \\ \mathrm{H} & -4.247600 & -4.305000 & -2.550500 \\ \mathrm{H} & -0.010500 & -4.798600 & -1.934100 \\ \mathrm{H} & -1.062800 & -6.220400 & -1.814000 \\ \mathrm{H} & -0.770300 & -5.443900 & -3.393700\end{array}$

Tomanik et al. "Development of an enantioselective synthesis of (-)-euonyminol." J. Org. Chem. 
Transition state TS-6:

C $\quad-0.516000 \quad-2.396600 \quad-2.004700$

$\begin{array}{llll}\text { C } & -0.238300 & -1.095100 & 0.039900\end{array}$

$\begin{array}{llll}\text { C } & -1.254700 & -1.970200 & -0.738100\end{array}$

$\begin{array}{llll}\text { C } & -1.609400 & -3.248400 & -0.004000\end{array}$

$\begin{array}{llll}\text { C } & -1.977500 & -2.998700 & 1.465500\end{array}$

$\begin{array}{llll}\text { C } & -0.952600 & -1.987900 & 2.089000\end{array}$

$\begin{array}{llll}\text { C } & -0.993600 & -0.639000 & 1.302900\end{array}$

$\begin{array}{llll}\mathrm{O} & 0.822900 & -1.957900 & 0.539300\end{array}$

$\begin{array}{llll}\text { C } & 0.531700 & -2.391500 & 1.894900\end{array}$

$\begin{array}{llll}\text { C } & 0.871800 & -3.875400 & 2.063300\end{array}$

$\begin{array}{llll}\text { C } & 1.485200 & -1.599800 & 2.814200\end{array}$

$\begin{array}{llll}\mathrm{O} & 1.108900 & -1.861500 & 4.173700\end{array}$

$\begin{array}{llll}\text { C } & 1.788400 & -1.093600 & 5.103600\end{array}$

$\begin{array}{llll}\mathrm{O} & 1.347000 & 0.248700 & 5.021400\end{array}$

$\begin{array}{llll}\text { C } & 2.084800 & 1.122800 & 5.851300\end{array}$

$\begin{array}{llll}\mathrm{O} & -0.355900 & 0.467800 & 1.930200\end{array}$

$\begin{array}{llll}\text { C } & -1.016200 & 0.959300 & 3.081100\end{array}$

$\begin{array}{llll}\mathrm{O} & -2.355100 & 1.311700 & 2.858300\end{array}$

$\begin{array}{llll}\text { C } & -2.536500 & 2.380800 & 1.940500\end{array}$

$\begin{array}{lrrr}\text { C } & 0.323900 & -0.137200 & -1.035300\end{array}$

$\begin{array}{llll}\text { C } & -0.053800 & 1.223500 & -1.038200\end{array}$

$\begin{array}{llll}\mathrm{O} & -2.571100 & -3.924800 & -0.823400\end{array}$

$\begin{array}{llll}\mathrm{O} & -1.451400 & -3.094300 & -2.823500\end{array}$

$\mathrm{H} \quad-1.960900 \quad-3.939600 \quad 2.026500$

$\mathrm{H} \quad-2.983000 \quad-2.583800 \quad 1.547900$

$\mathrm{H}-1.184800 \quad-1.855500 \quad 3.147000$

$\mathrm{H} \quad-2.023300 \quad-0.359000 \quad 1.091300$

$\mathrm{H} \quad-0.695300 \quad-3.845200 \quad 0.004400$

$\begin{array}{llll}\mathrm{H} & -0.708600 & 1.530600 & -0.229700\end{array}$

$\begin{array}{llll}\mathrm{H} & -1.029400 & 0.219300 & 3.885700\end{array}$

$\mathrm{H} \quad-0.412700 \quad 1.823400 \quad 3.388500$

$\begin{array}{llll}\mathrm{H} & -3.568200 & 2.724100 & 2.055200\end{array}$

$\begin{array}{llll}\mathrm{H} & -2.380300 & 2.061400 & 0.901600\end{array}$

$\mathrm{H} \quad-1.852300 \quad 3.216300 \quad 2.152600$

$\begin{array}{llll}\mathrm{H} & 1.670300 & 2.123900 & 5.710100\end{array}$

$\begin{array}{llll}\mathrm{H} & 1.998700 & 0.849800 & 6.915800\end{array}$

$\mathrm{H} \quad 3.153800 \quad 1.138400 \quad 5.582700$

$\begin{array}{llll}\mathrm{H} & 2.884000 & -1.132400 & 4.939200\end{array}$

$\mathrm{H} \quad 1.556400 \quad-1.515800 \quad 6.093400$

$\mathrm{H} \quad 2.515900 \quad-1.947100 \quad 2.641300$

$\mathrm{H} \quad 1.425500 \quad-0.536300 \quad 2.596600$

$\mathrm{H} \quad 0.847500 \quad-4.124700 \quad 3.128500$

$\begin{array}{llll}\mathrm{H} & 1.887200 & -4.051300 & 1.692400\end{array}$

$\begin{array}{llll}\mathrm{H} & 0.200000 & -4.553500 & 1.536600\end{array}$

$\begin{array}{llll}\text { C } & -2.498400 & -1.152800 & -1.177100\end{array}$

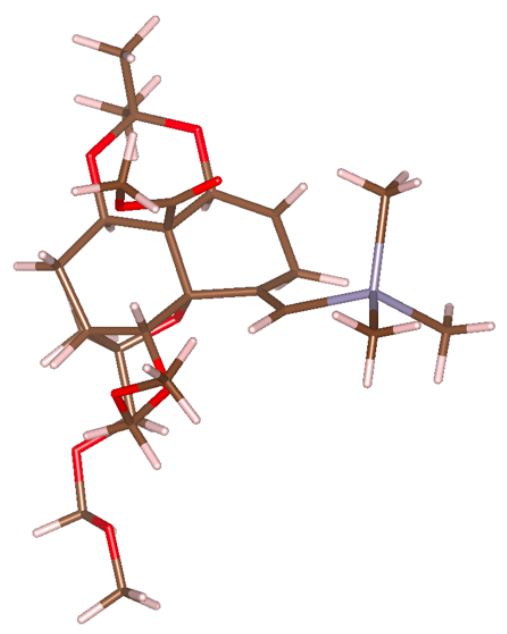




$\begin{array}{lrrr}\mathrm{O} & -3.526600 & -1.257500 & -0.302900 \\ \mathrm{O} & -2.582400 & -0.468000 & -2.171700 \\ \mathrm{C} & -4.704100 & -0.513800 & -0.650900 \\ \mathrm{H} & -4.478900 & 0.554900 & -0.712600 \\ \mathrm{H} & -5.419100 & -0.712200 & 0.148000 \\ \mathrm{H} & -5.095700 & -0.849200 & -1.615000 \\ \mathrm{H} & 0.292500 & -3.079800 & -1.705600 \\ \mathrm{C} & 0.119400 & -1.135700 & -2.542700 \\ \mathrm{H} & -0.319800 & -0.610200 & -3.377100 \\ \mathrm{C} & 1.400400 & -0.722100 & -1.925900 \\ \mathrm{H} & 2.020000 & -1.497000 & -1.475700 \\ \mathrm{H} & 1.989300 & 0.020200 & -2.464400 \\ \mathrm{Sn} & 0.178000 & 2.677900 & -2.592400 \\ \mathrm{C} & -0.857600 & 1.970500 & -4.382500 \\ \mathrm{C} & -0.776200 & 4.485800 & -1.829700 \\ \mathrm{C} & 2.272700 & 3.080300 & -3.071700 \\ \mathrm{H} & -0.187300 & 1.360500 & -4.996400 \\ \mathrm{H} & -1.215700 & 2.810100 & -4.986200 \\ \mathrm{H} & -0.759500 & 5.283300 & -2.578600 \\ \mathrm{H} & -0.258400 & 4.838300 & -0.932400 \\ \mathrm{H} & 2.733800 & 2.201500 & -3.533700 \\ \mathrm{H} & 2.349200 & 3.918800 & -3.771100 \\ \mathrm{H} & 2.828100 & 3.327900 & -2.162300 \\ \mathrm{H} & -1.818700 & 4.274500 & -1.570800 \\ \mathrm{H} & -1.707400 & 1.357200 & -4.069900 \\ \mathrm{C} & -2.087200 & -4.215000 & -2.157500 \\ \mathrm{C} & -1.137200 & -5.419400 & -2.130100 \\ \mathrm{C} & -3.339100 & -4.489400 & -2.975500 \\ \mathrm{H} & -0.228900 & -5.221300 & -1.554800 \\ \mathrm{H} & -0.840000 & -5.672300 & -3.151800 \\ \mathrm{H} & -1.645800 & -6.276300 & -1.678900 \\ \mathrm{H} & -3.068100 & -4.722700 & -4.008700 \\ \mathrm{H} & -3.970500 & -3.597400 & -2.965700 \\ \mathrm{H} & -3.892900 & -5.328300 & -2.545000\end{array}$

Tomanik et al. "Development of an enantioselective synthesis of (-)-euonyminol." J. Org. Chem. 
Transition state TS-7:

C $-1.054500 \quad-2.531600 \quad-2.122400$

$\begin{array}{llll}\text { C } & -0.748000 & -1.065200 & -0.232200\end{array}$

$\begin{array}{llll}\text { C } & -1.674800 & -2.184500 & -0.775000\end{array}$

$\begin{array}{llll}\text { C } & -1.554100 & -3.440100 & 0.079400\end{array}$

$\begin{array}{llll}\text { C } & -1.734300 & -3.140000 & 1.579600\end{array}$

$\begin{array}{llll}\text { C } & -0.890700 & -1.872500 & 1.968400\end{array}$

$\begin{array}{llll}\text { C } & -1.329400 & -0.633100 & 1.119300\end{array}$

$\begin{array}{lrrr}\mathrm{O} & 0.534100 & -1.674800 & 0.117700\end{array}$

$\begin{array}{llll}\text { C } & 0.589100 & -2.003700 & 1.534100\end{array}$

$\begin{array}{llll}\text { C } & 1.256100 & -3.371100 & 1.724900\end{array}$

$\begin{array}{llll}\text { C } & 1.529200 & -0.967300 & 2.195900\end{array}$

$\begin{array}{llll}\mathrm{O} & 1.653800 & -1.240300 & 3.599600\end{array}$

C $\quad 1.371600 \quad-0.193200 \quad 4.454600$

$\begin{array}{llll}\mathrm{O} & -0.032900 & -0.031800 & 4.591400\end{array}$

$\begin{array}{llll}\text { C } & -0.366100 & 0.997600 & 5.501200\end{array}$

$\begin{array}{llll}\mathrm{O} & -0.777600 & 0.605500 & 1.558500\end{array}$

$\begin{array}{llll}\text { C } & -1.680700 & 1.397900 & 2.289000\end{array}$

$\begin{array}{llll}\mathrm{O} & -2.746300 & 1.876800 & 1.513300\end{array}$

$\begin{array}{llll}\text { C } & -2.325000 & 2.699300 & 0.428900\end{array}$

$\begin{array}{llll}\text { C } & -0.451100 & -0.109500 & -1.398600\end{array}$

$\begin{array}{llll}\text { C } & 0.709200 & 0.694600 & -1.341700\end{array}$

$\begin{array}{llll}\mathrm{O} & -2.444300 & -4.389800 & -0.519400\end{array}$

$\begin{array}{llll}\mathrm{O} & -1.851300 & -3.552100 & -2.740500\end{array}$

$\mathrm{H} \quad-1.401800 \quad-3.997700 \quad 2.174100$

$\mathrm{H} \quad-2.783500 \quad-2.966700 \quad 1.817100$

$\mathrm{H} \quad-0.979300 \quad-1.674800 \quad 3.038400$

$\mathrm{H} \quad-2.409200 \quad-0.527900 \quad 1.076200$

$\mathrm{H} \quad-0.535700 \quad-3.802300 \quad-0.050200$

$\begin{array}{llll}\mathrm{H} & 1.416000 & 0.441500 & -0.556700\end{array}$

$\begin{array}{llll}\mathrm{H} & -2.137800 & 0.831500 & 3.107800\end{array}$

$\begin{array}{llll}\mathrm{H} & -1.071900 & 2.220800 & 2.690900\end{array}$

$\begin{array}{llll}\mathrm{H} & -3.230000 & 3.026200 & -0.088400\end{array}$

$\begin{array}{llll}\mathrm{H} & -1.677500 & 2.152400 & -0.265000\end{array}$

$\begin{array}{llll}\mathrm{H} & -1.779100 & 3.584900 & 0.791900\end{array}$

$\begin{array}{llll}\mathrm{H} & -1.456600 & 1.043100 & 5.552200\end{array}$

$\begin{array}{llll}\mathrm{H} & 0.028700 & 0.796000 & 6.510300\end{array}$

$\begin{array}{llll}\mathrm{H} & 0.014500 & 1.978100 & 5.170200\end{array}$

$\begin{array}{llll}\mathrm{H} & 1.813500 & 0.758300 & 4.101400\end{array}$

$\begin{array}{llll}\mathrm{H} & 1.812500 & -0.454600 & 5.429200\end{array}$

$\begin{array}{llll}\mathrm{H} & 2.521400 & -1.075000 & 1.738800\end{array}$

$\begin{array}{llll}\mathrm{H} & 1.163000 & 0.041600 & 2.024800\end{array}$

$\mathrm{H} \quad 1.452900 \quad-3.524500 \quad 2.789700$

$\mathrm{H} \quad 2.214800 \quad-3.373500 \quad 1.195100$

$\begin{array}{llll}\mathrm{H} & 0.671600 & -4.214700 & 1.358700\end{array}$

$\begin{array}{llll}\text { C } & -3.192600 & -1.878000 & -0.952900\end{array}$

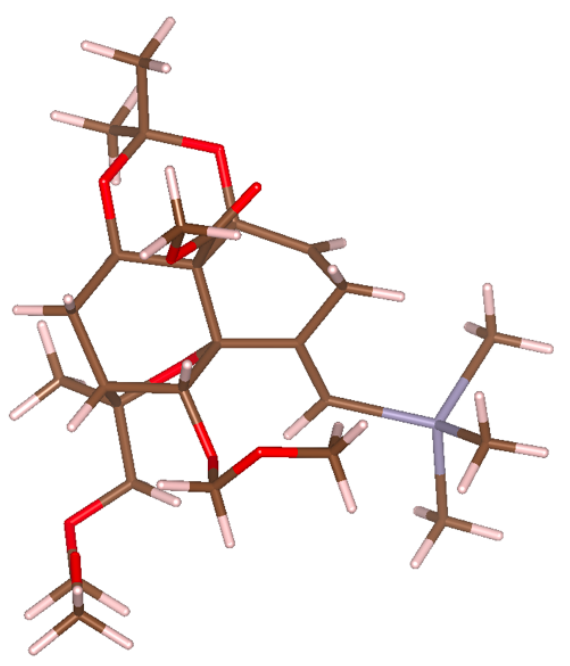

Tomanik et al. "Development of an enantioselective synthesis of (-)-euonyminol." J. Org. Chem. 


$\begin{array}{lrrr}\mathrm{O} & -3.859600 & -1.792700 & 0.222400 \\ \mathrm{O} & -3.776000 & -1.763400 & -2.007100 \\ \mathrm{C} & -5.279000 & -1.599000 & 0.109200 \\ \mathrm{H} & -5.498100 & -0.674300 & -0.430800 \\ \mathrm{H} & -5.645300 & -1.543700 & 1.134600 \\ \mathrm{H} & -5.733000 & -2.439800 & -0.421900 \\ \mathrm{H} & -0.059300 & -2.949700 & -1.919000 \\ \mathrm{C} & -0.795200 & -1.222500 & -2.829900 \\ \mathrm{C} & -1.493600 & 0.043000 & -2.478500 \\ \mathrm{C} & -3.345000 & -5.346300 & -2.475100 \\ \mathrm{C} & -0.883300 & -5.644700 & -1.930600 \\ \mathrm{Sn} & 1.200100 & 2.347100 & -2.615700 \\ \mathrm{C} & -0.360900 & 3.872700 & -2.464500 \\ \mathrm{C} & 3.105500 & 3.156500 & -1.934900 \\ \mathrm{C} & 1.348300 & 1.711600 & -4.701900 \\ \mathrm{H} & -1.334600 & 3.442100 & -2.718000 \\ \mathrm{H} & -0.158800 & 4.705100 & -3.145600 \\ \mathrm{H} & 3.396200 & 4.020300 & -2.540200 \\ \mathrm{H} & 3.888200 & 2.395900 & -2.013100 \\ \mathrm{H} & 0.403200 & 1.270600 & -5.034000 \\ \mathrm{H} & 1.580600 & 2.563800 & -5.348100 \\ \mathrm{H} & 2.138600 & 0.962800 & -4.811100 \\ \mathrm{H} & 3.028900 & 3.472200 & -0.890000 \\ \mathrm{H} & -0.407300 & 4.258100 & -1.441500 \\ \mathrm{H} & -0.036800 & -1.190700 & -3.604600 \\ \mathrm{H} & -1.275600 & 0.862300 & -3.162400 \\ \mathrm{H} & -2.546000 & 0.030700 & -2.225000 \\ \mathrm{C} & -2.093900 & -4.702900 & -1.898700 \\ \mathrm{H} & -3.171900 & -5.629200 & -3.517000 \\ \mathrm{H} & -3.614000 & -6.234600 & -1.896200 \\ \mathrm{H} & -4.160700 & -4.620700 & -2.435400 \\ \mathrm{H} & 0.024000 & -5.170200 & -1.546600 \\ \mathrm{H} & -1.088800 & -6.532100 & -1.324700 \\ \mathrm{H} & -0.690000 & -5.949100 & -2.963100\end{array}$

Tomanik et al. "Development of an enantioselective synthesis of (-)-euonyminol." J. Org. Chem. 
Catalog of nuclear magnetic resonance spectra.

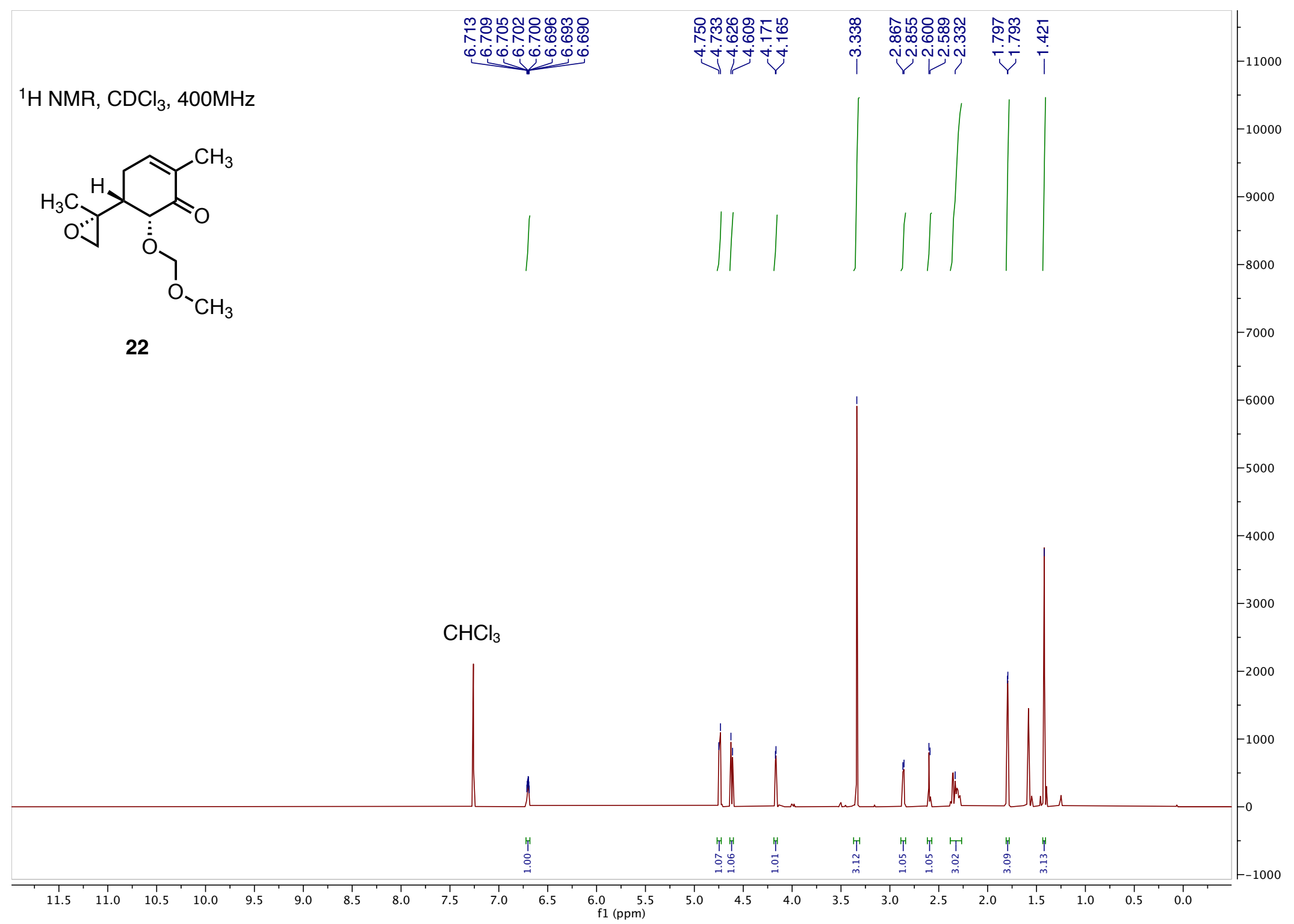




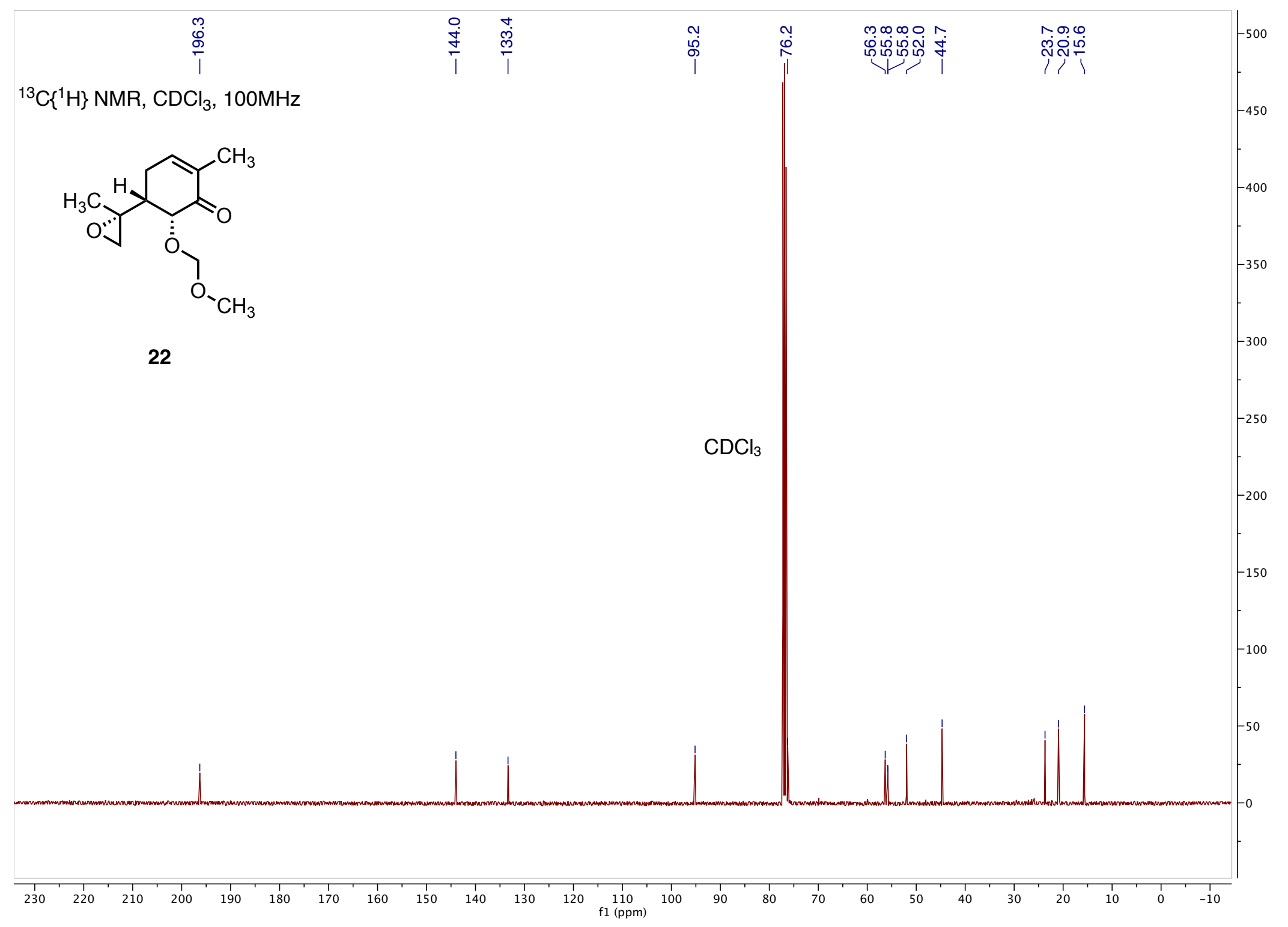




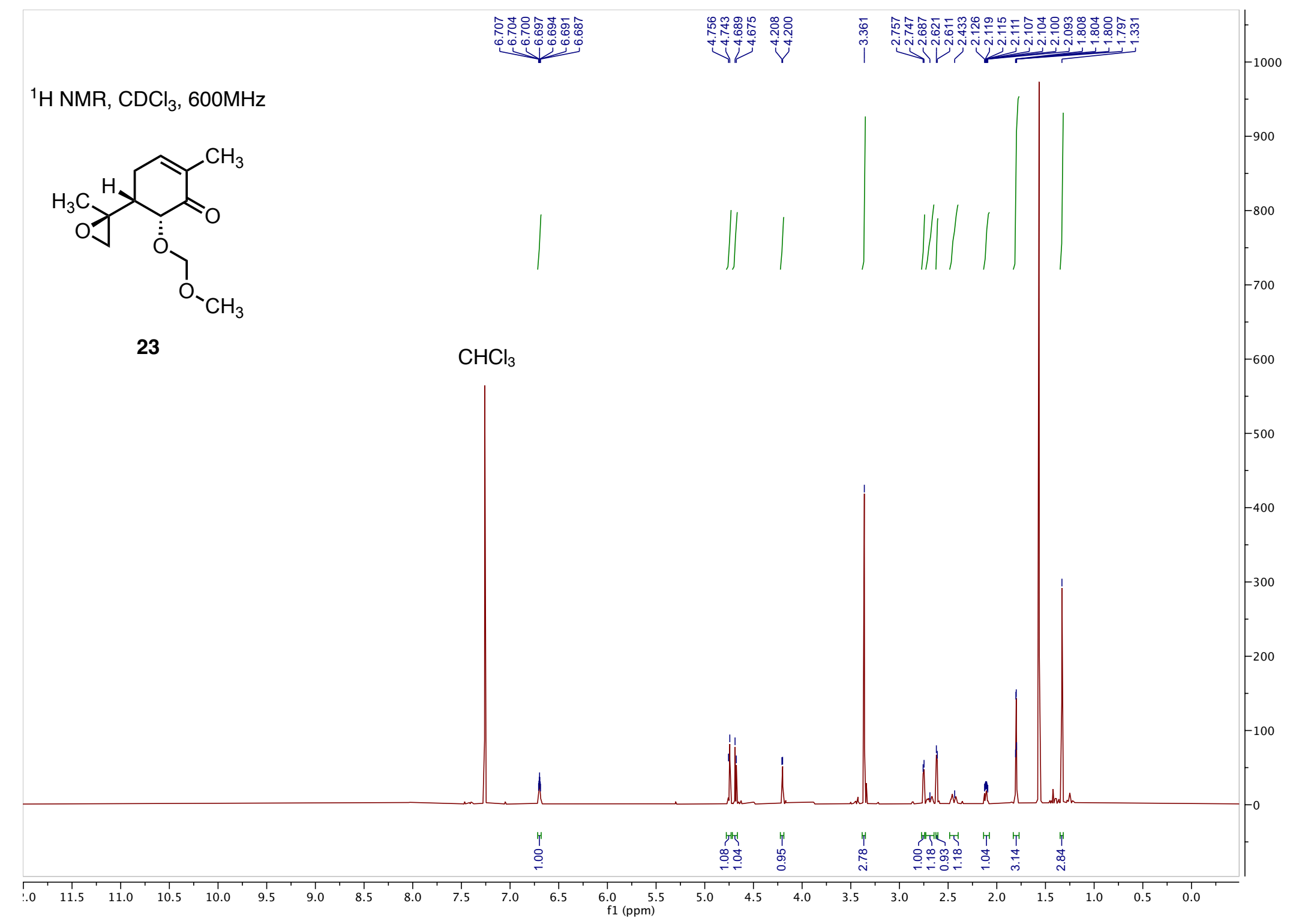




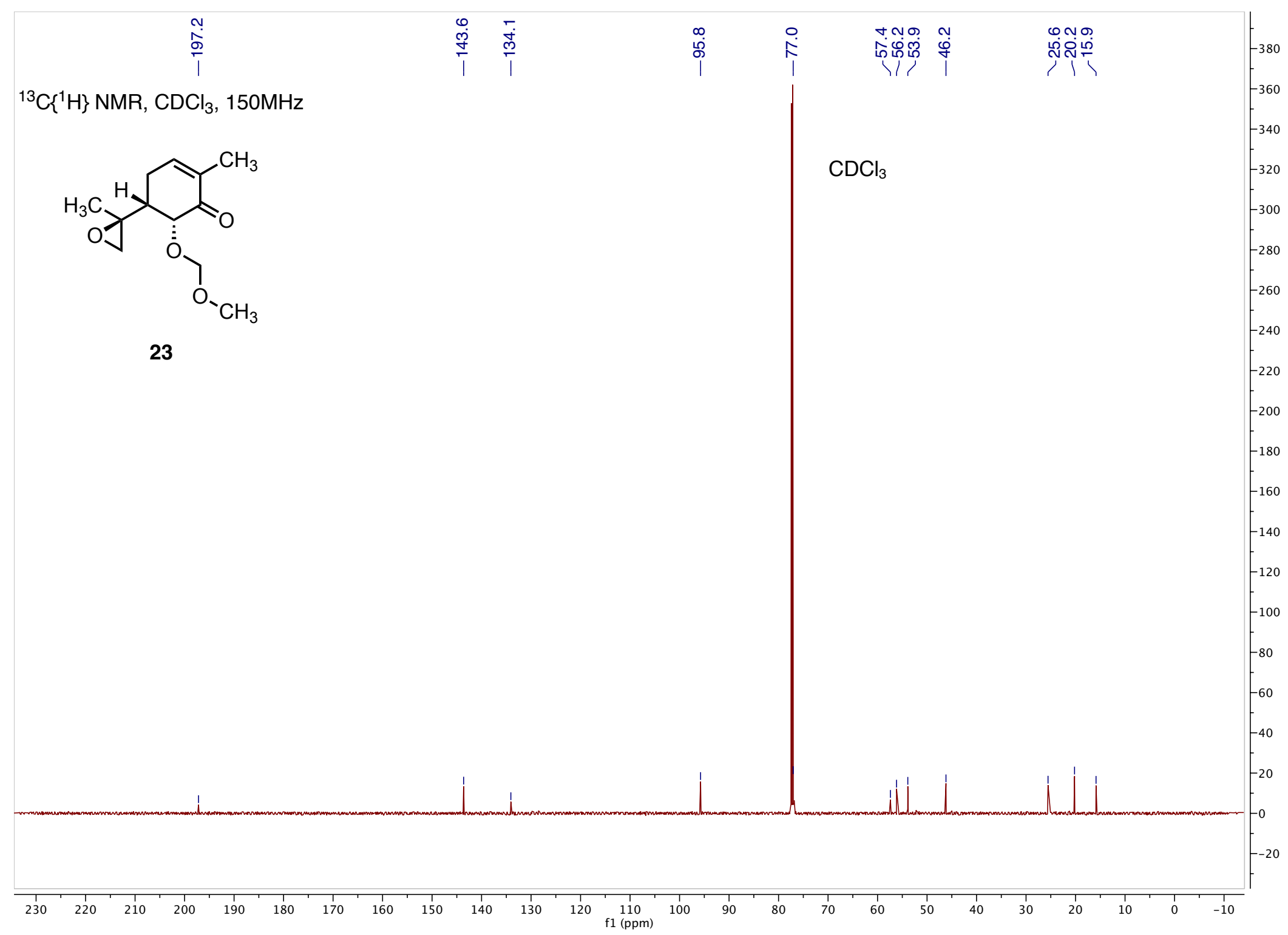




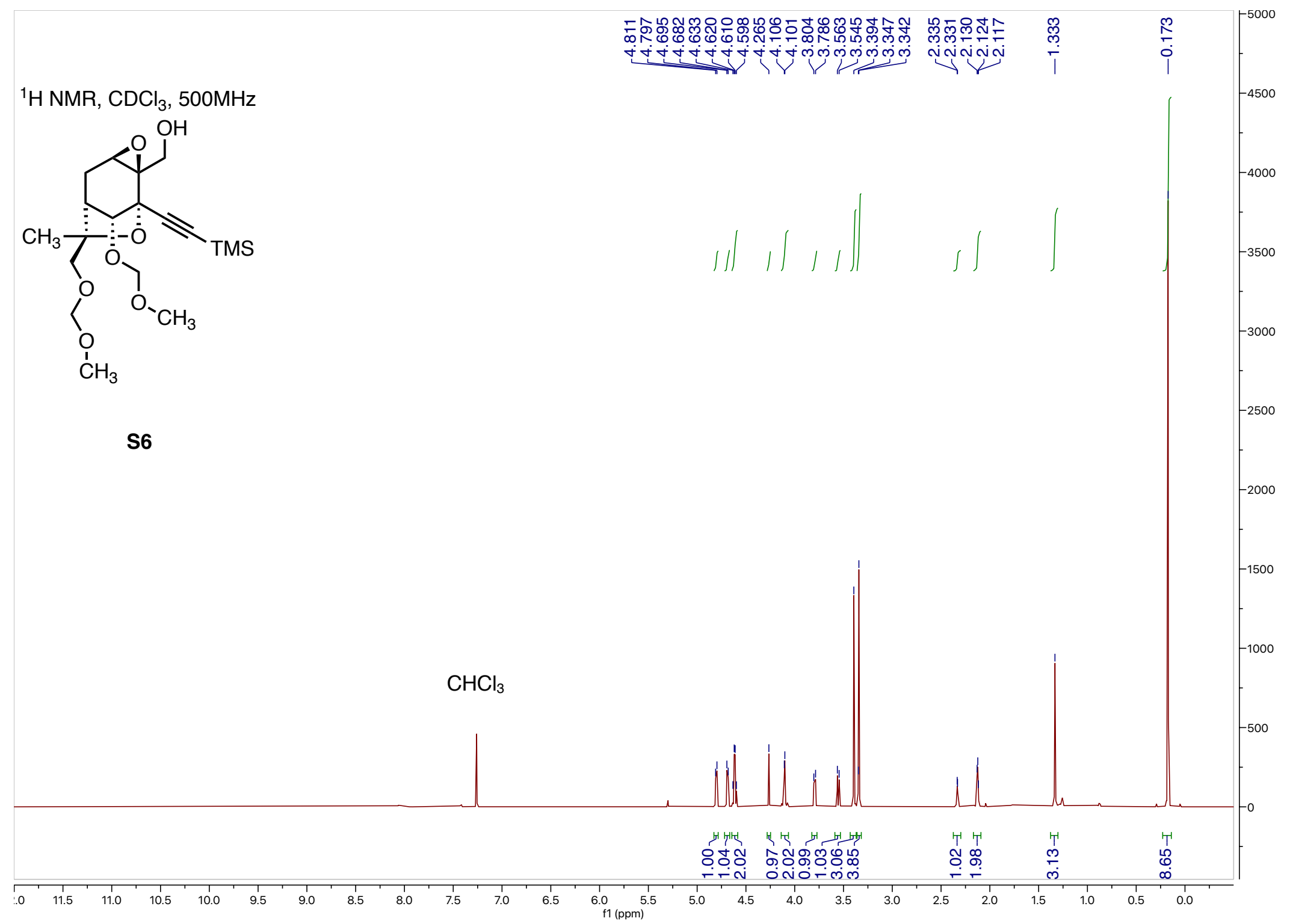




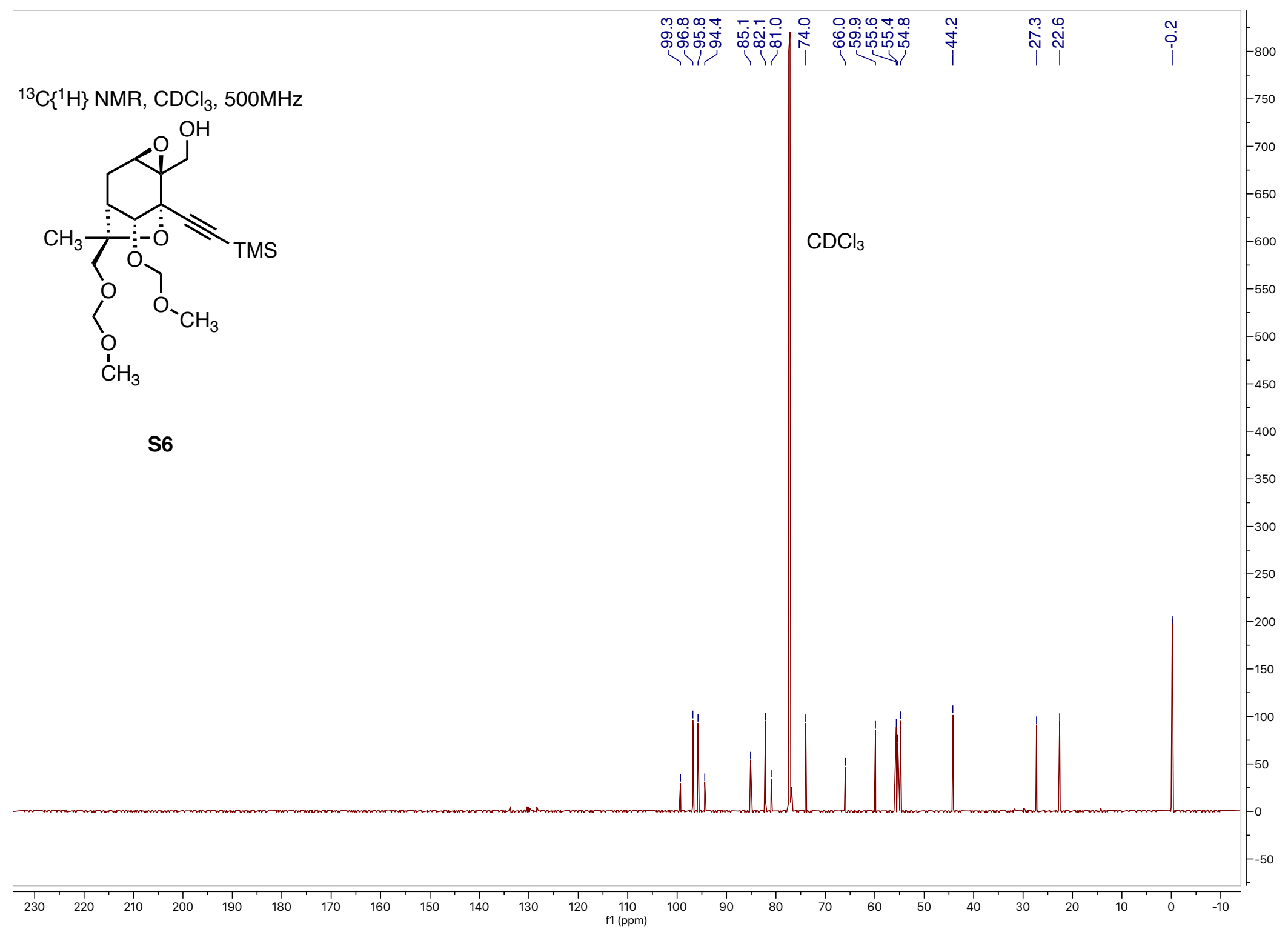




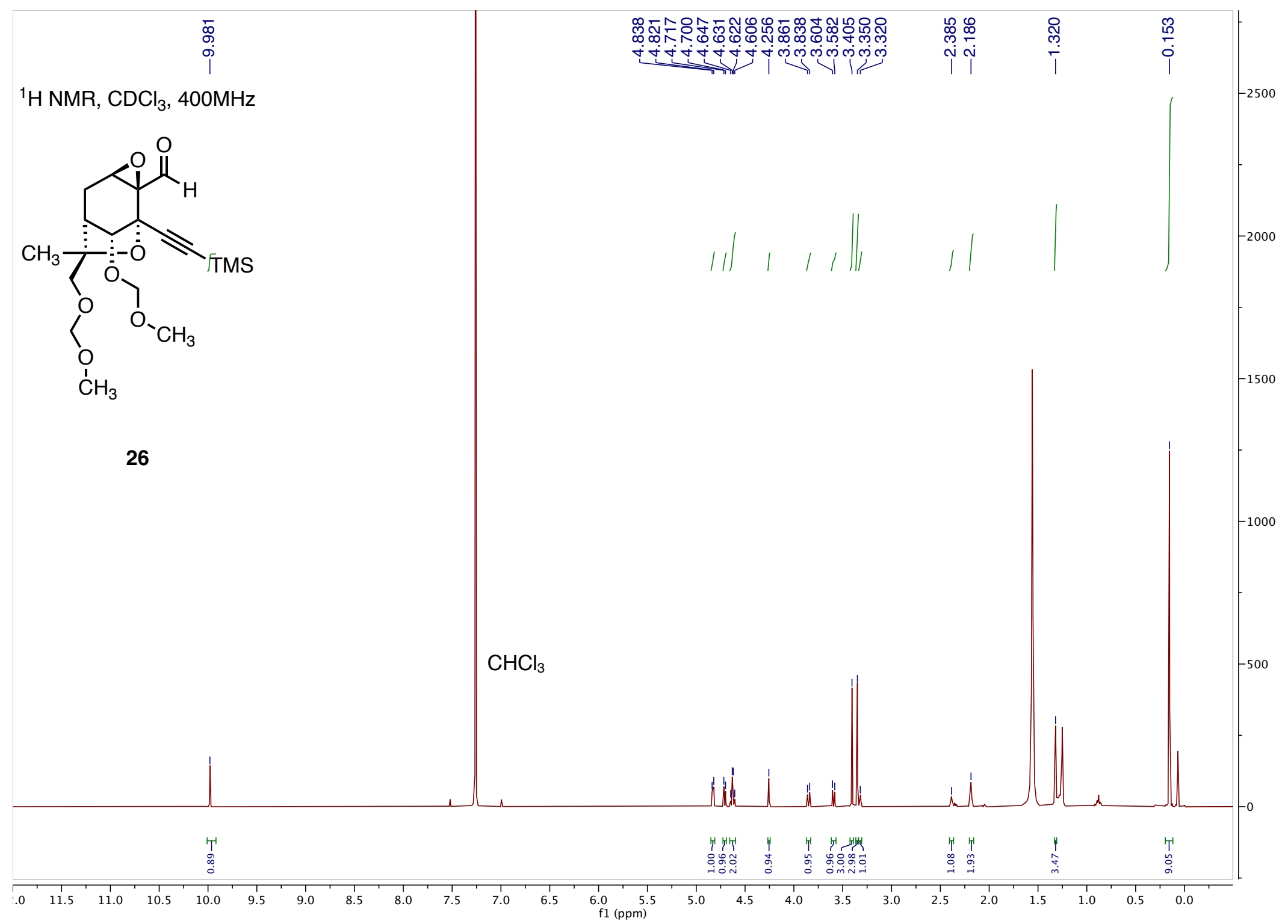




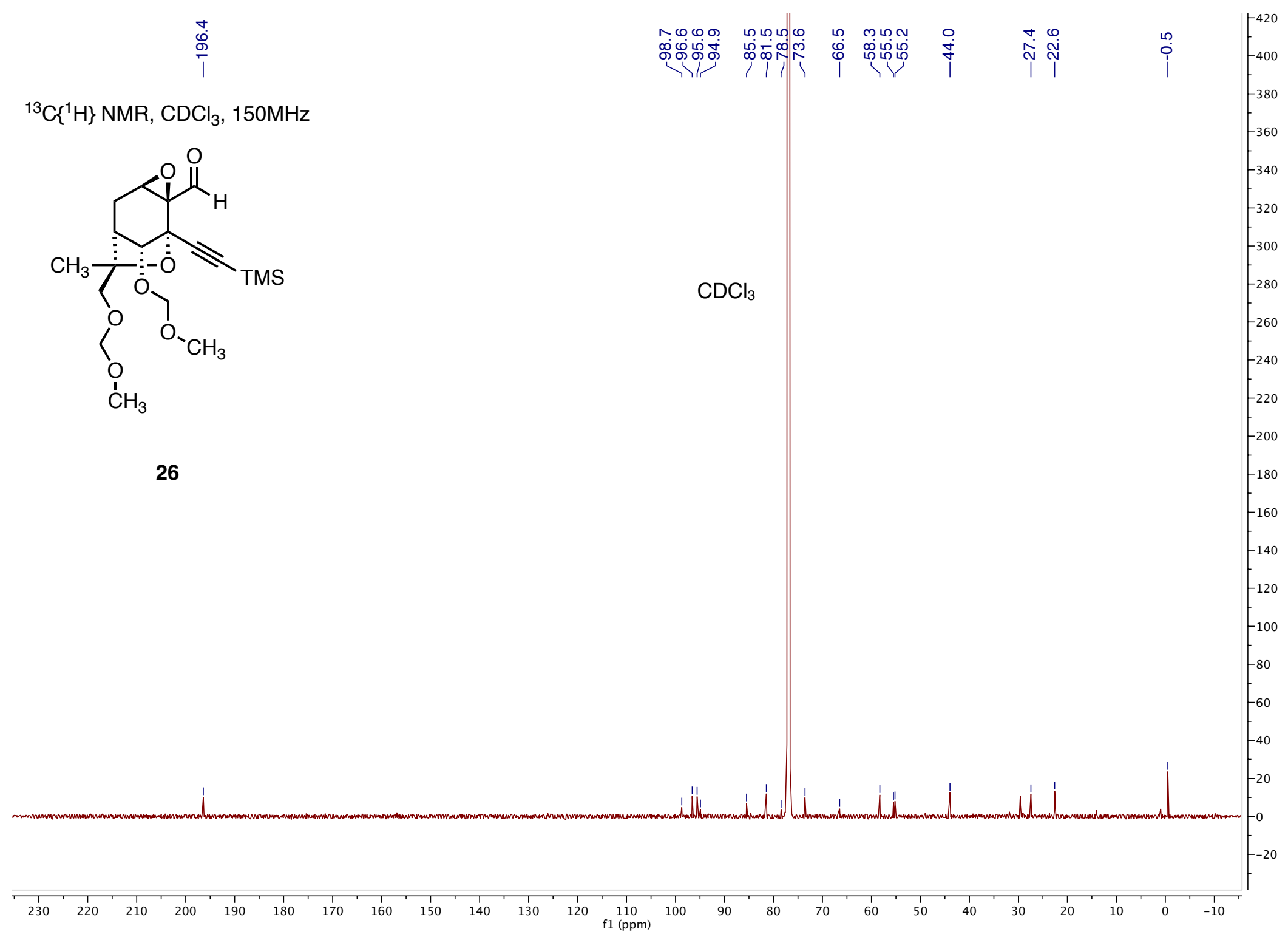




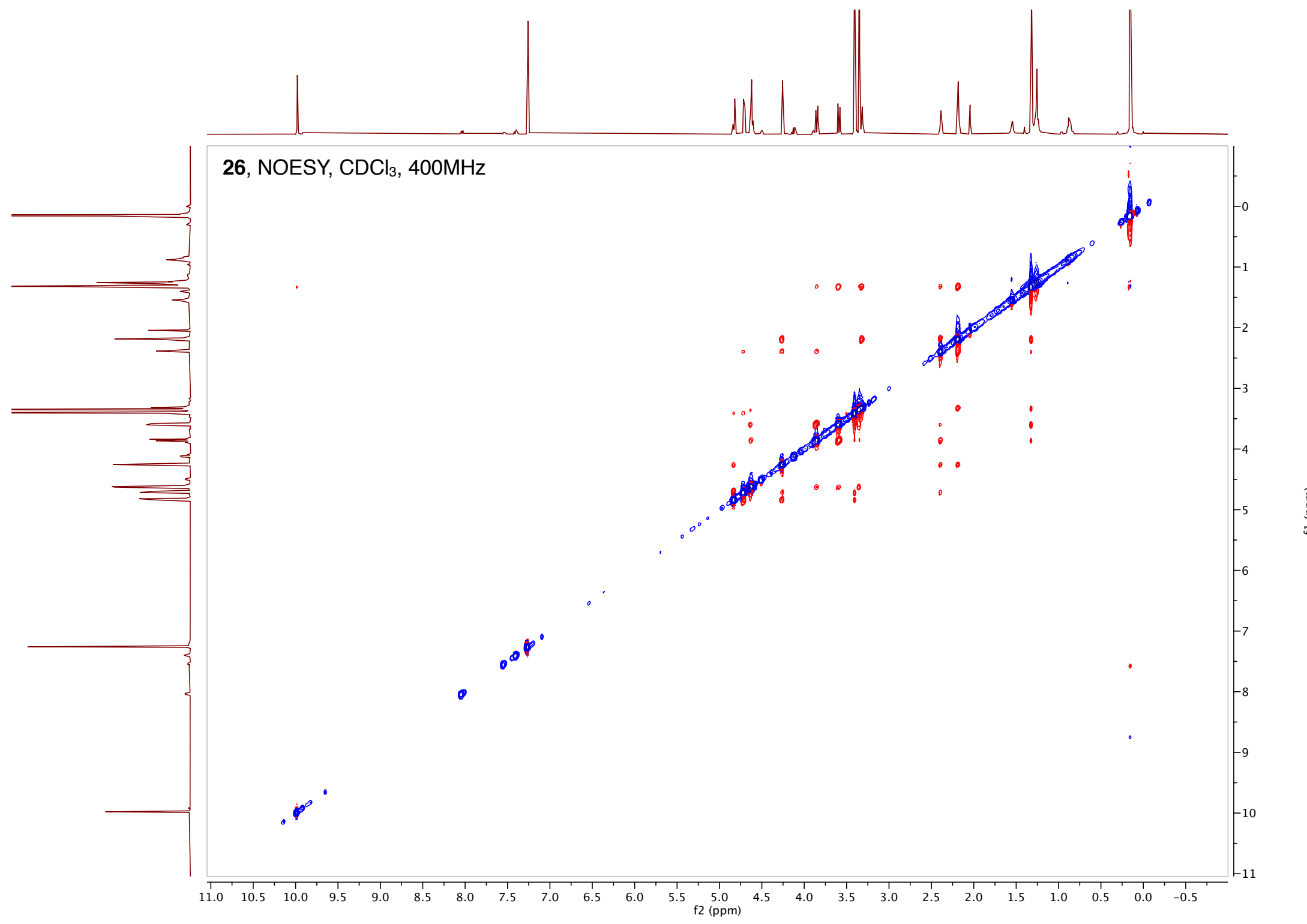




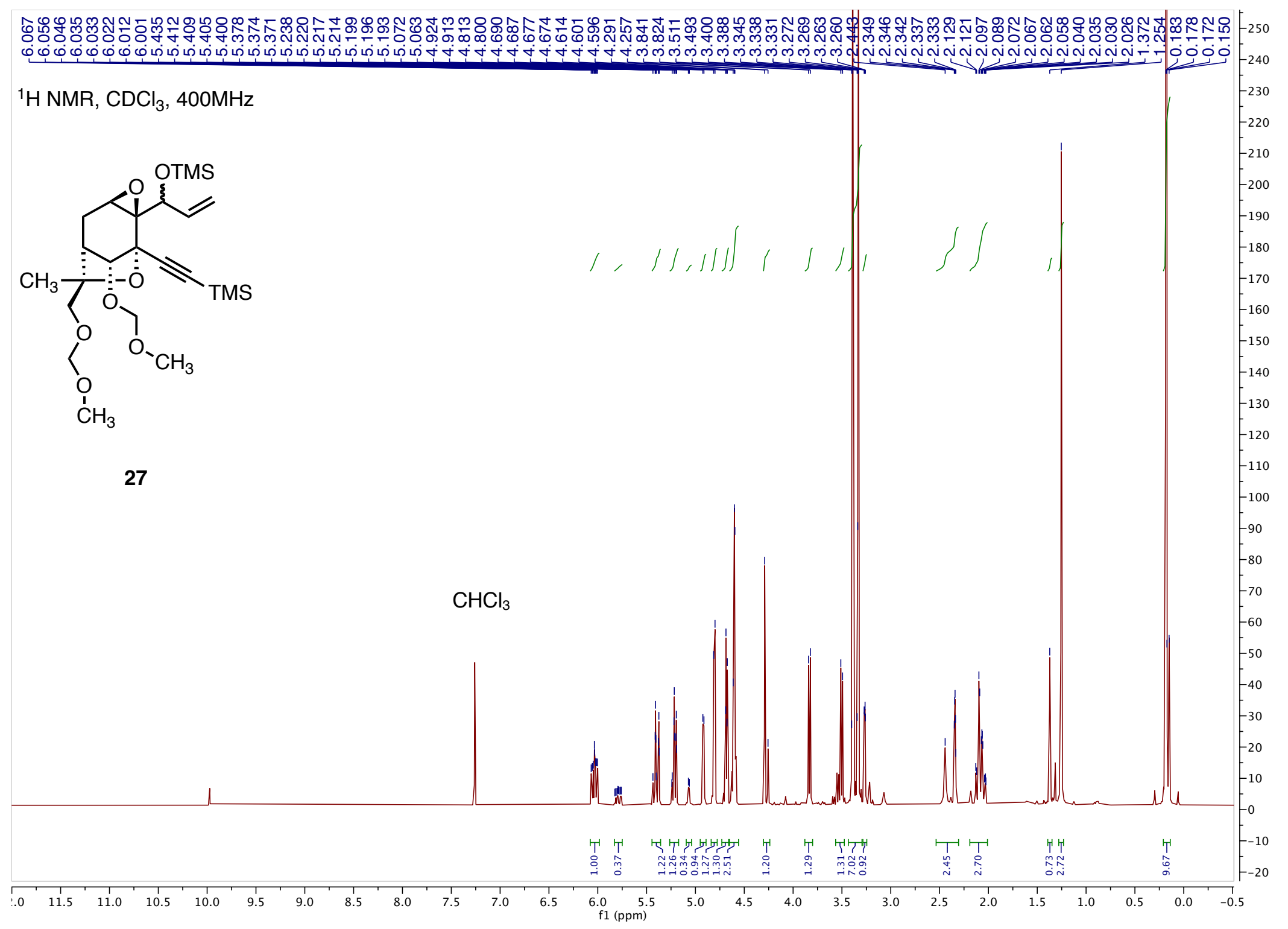




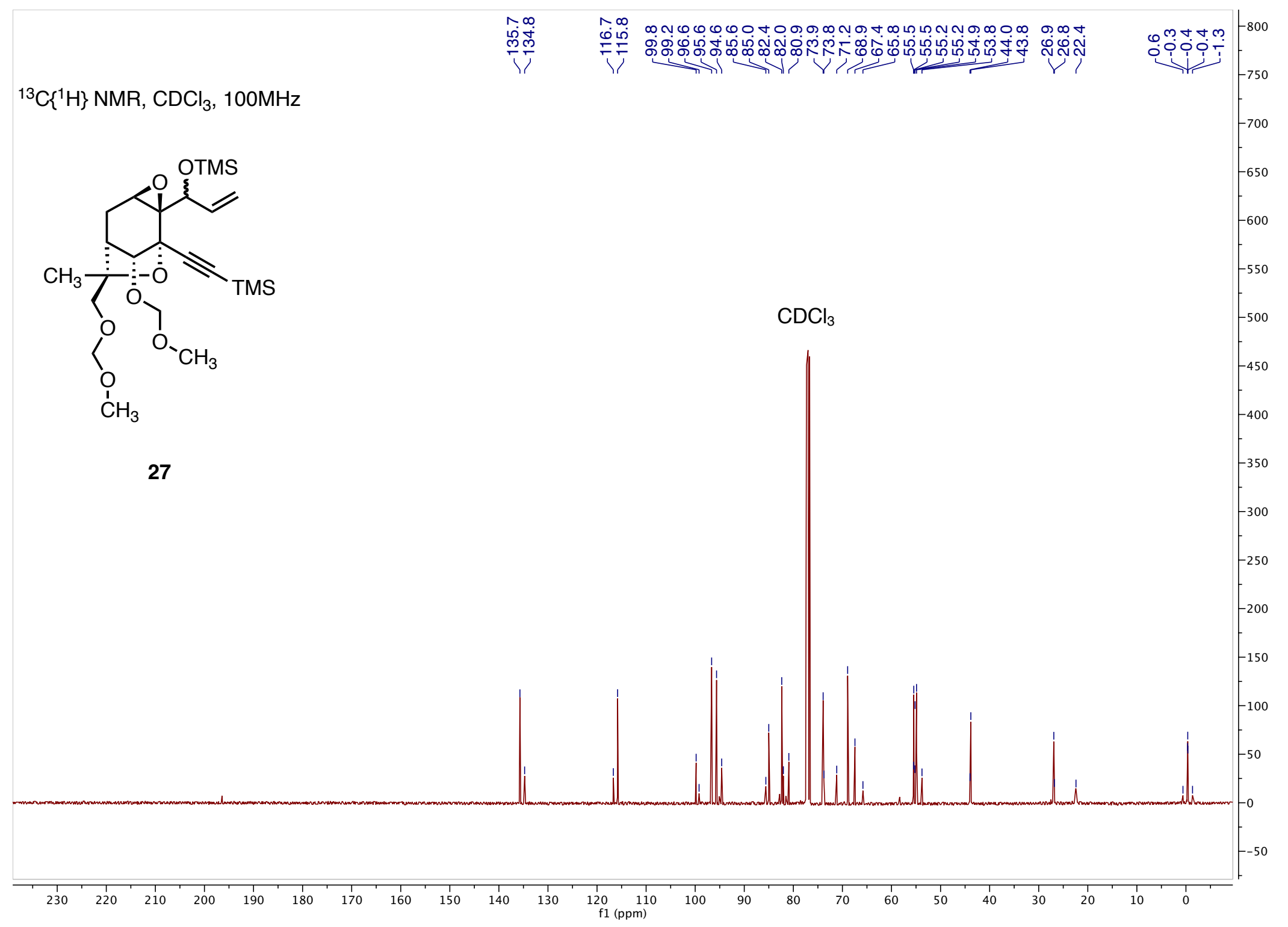




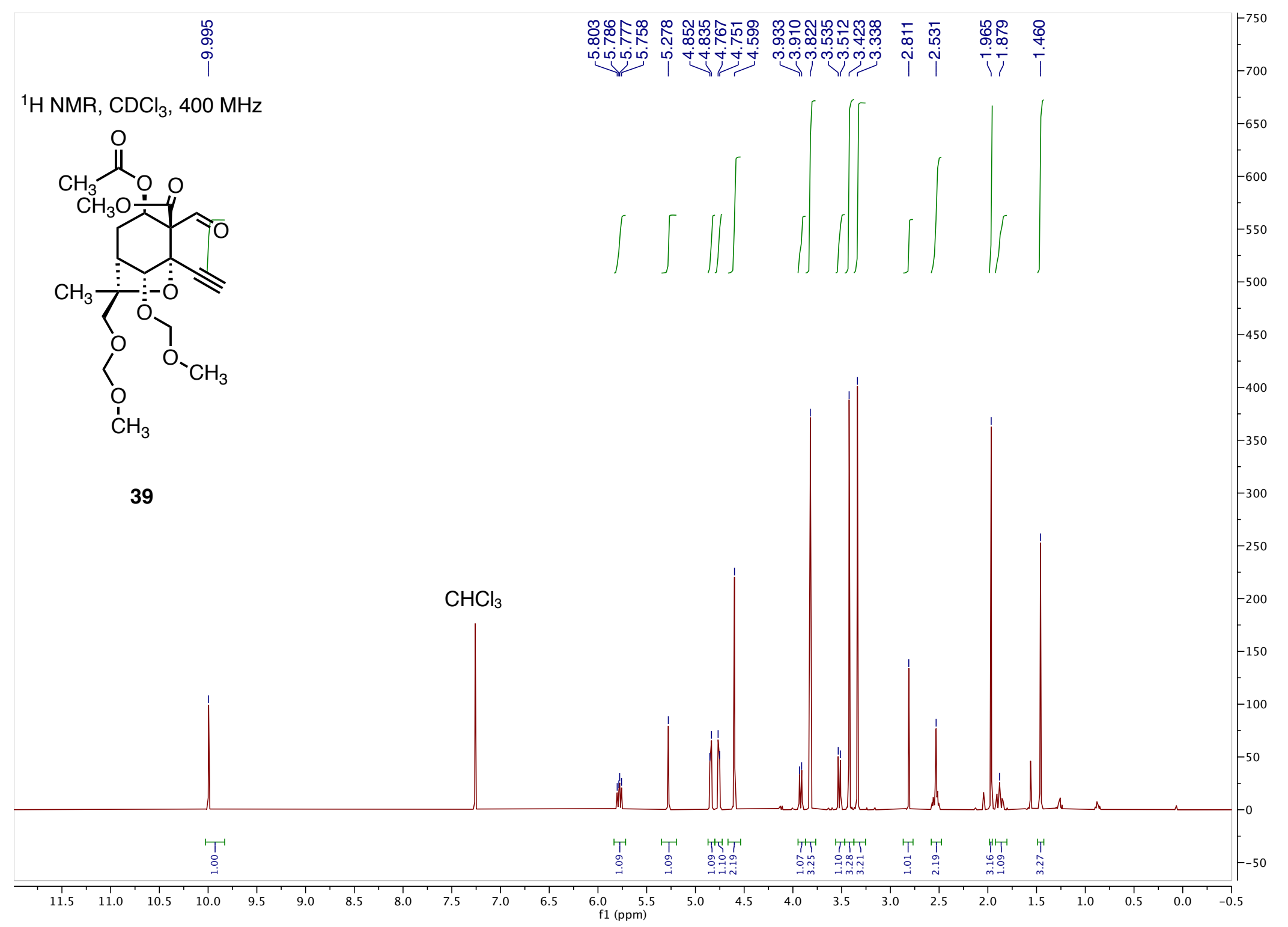




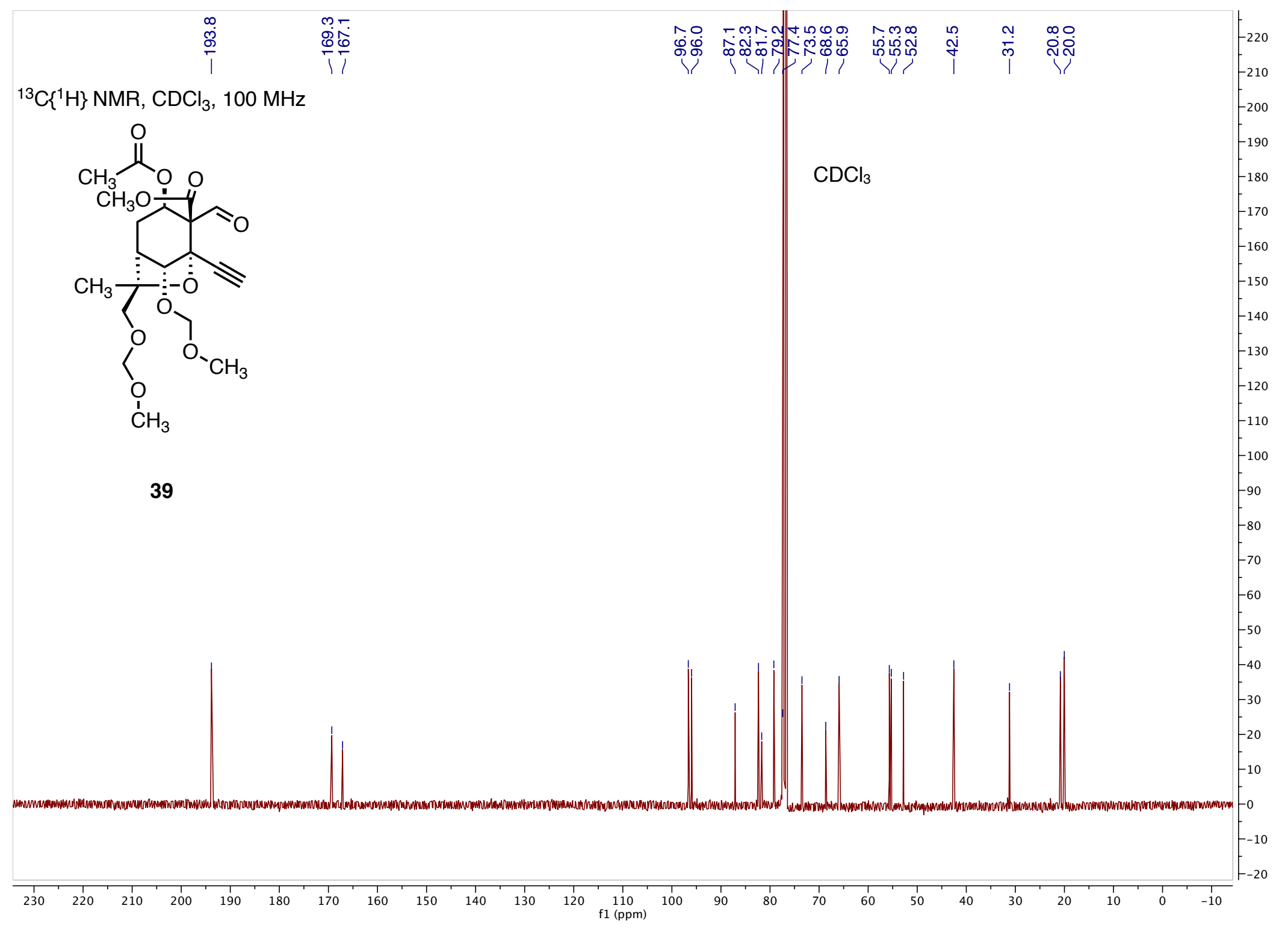




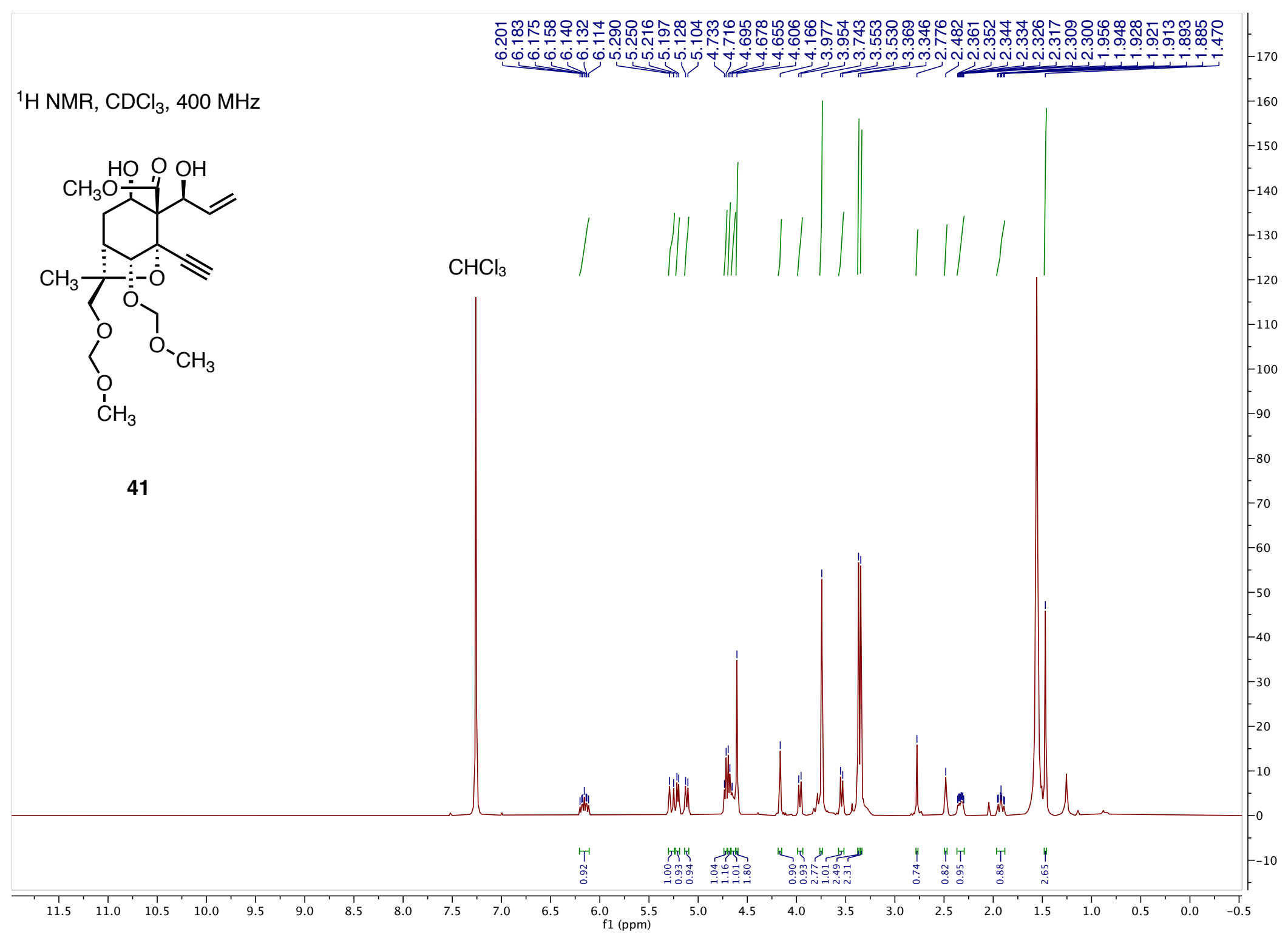




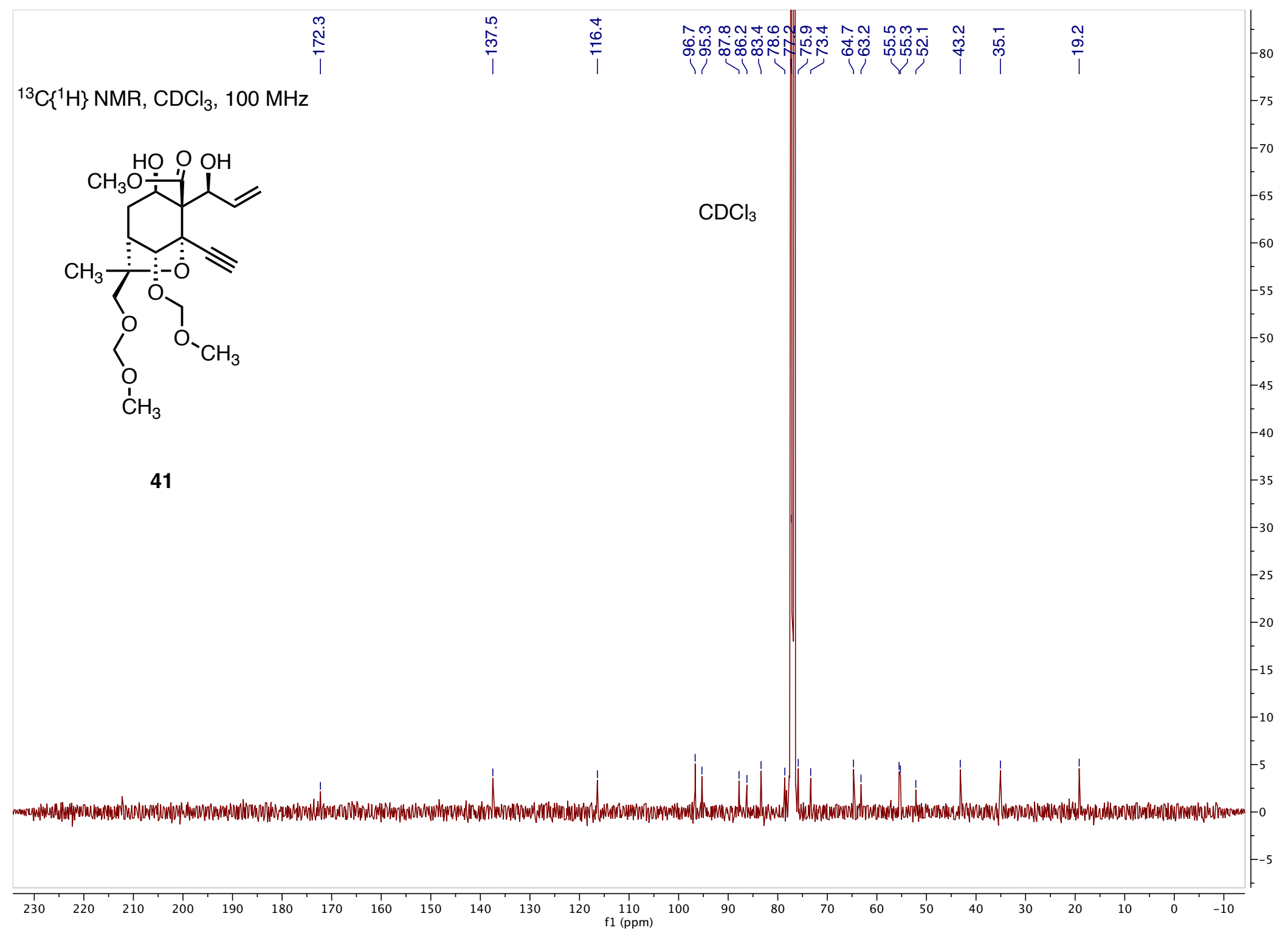




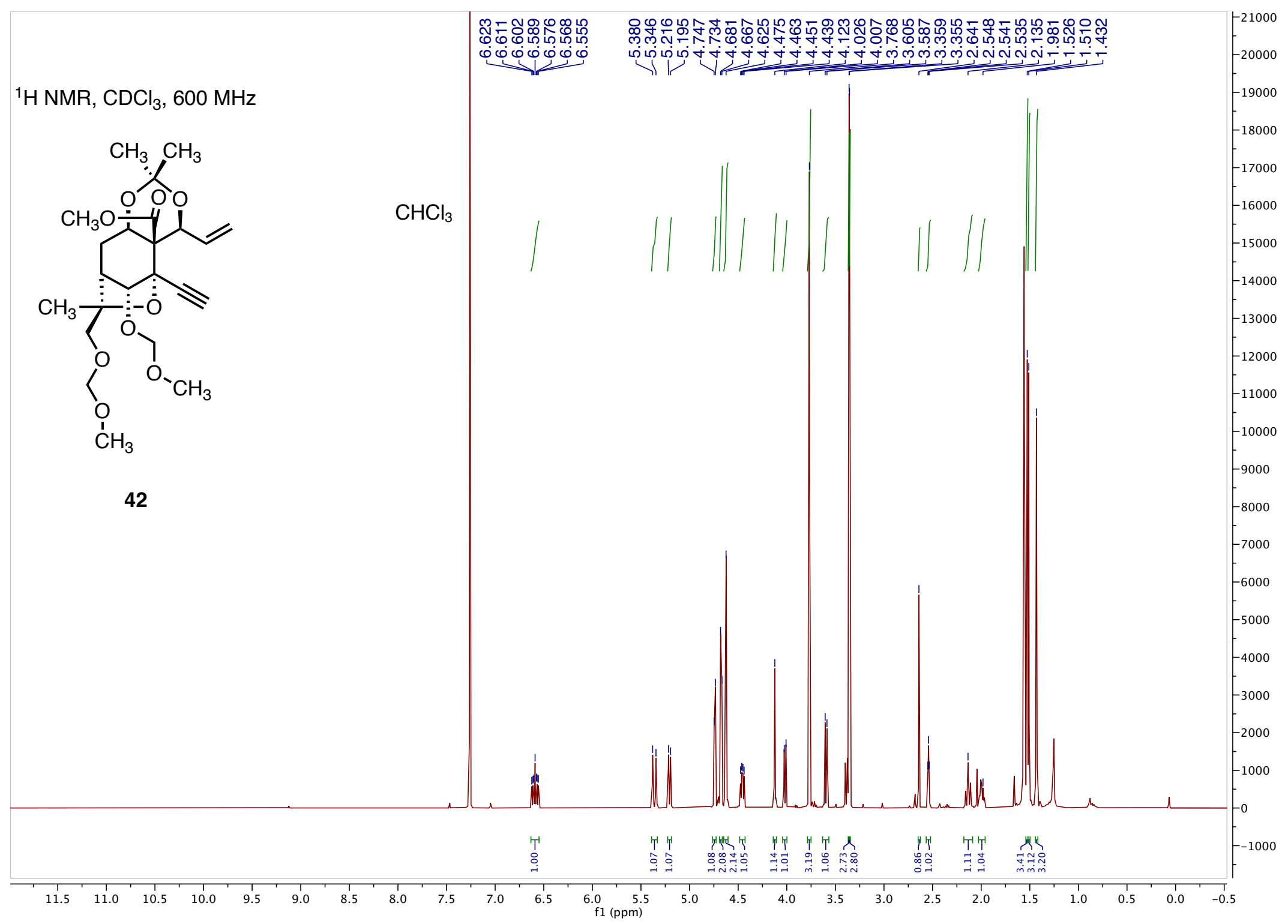




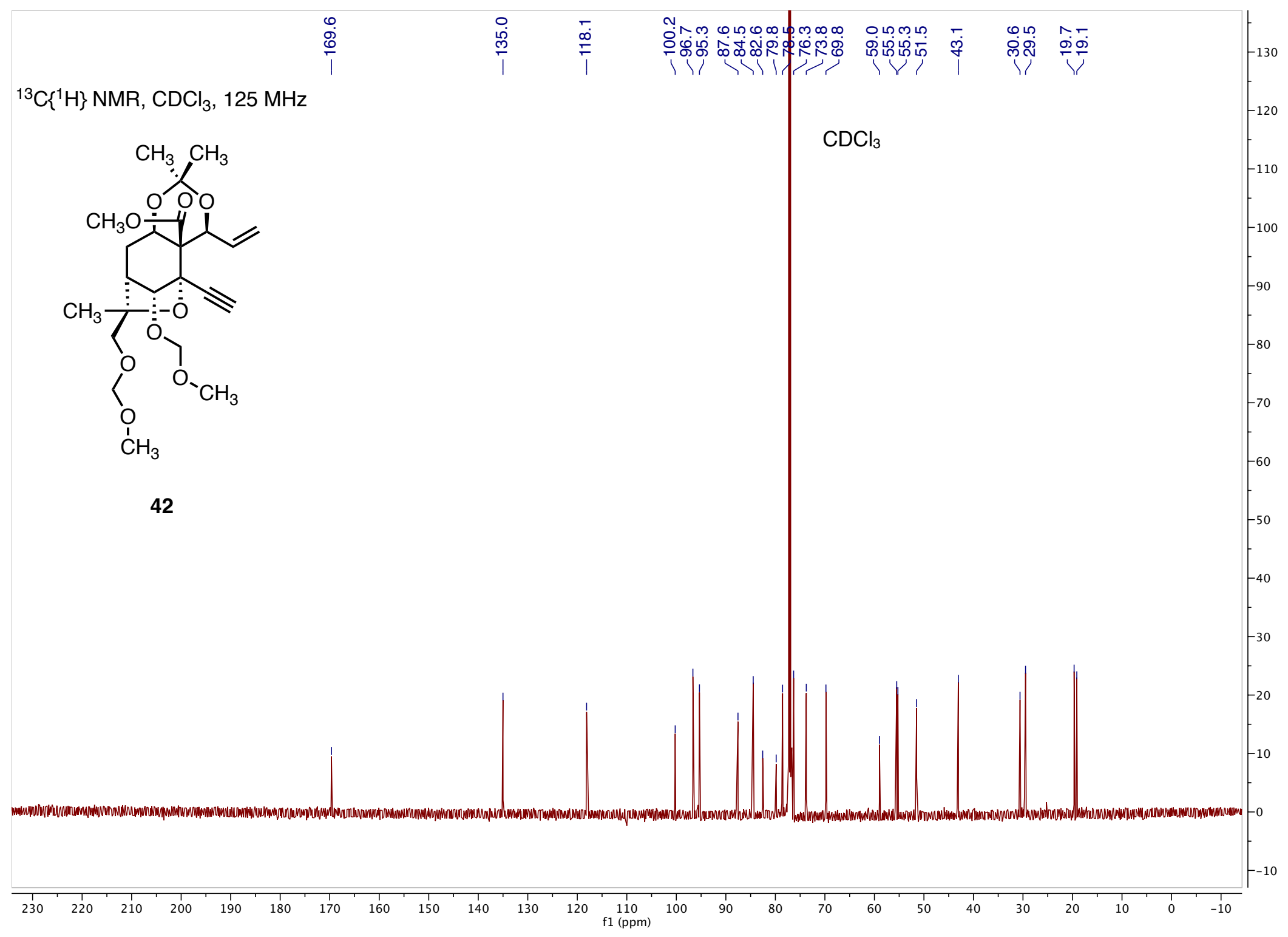




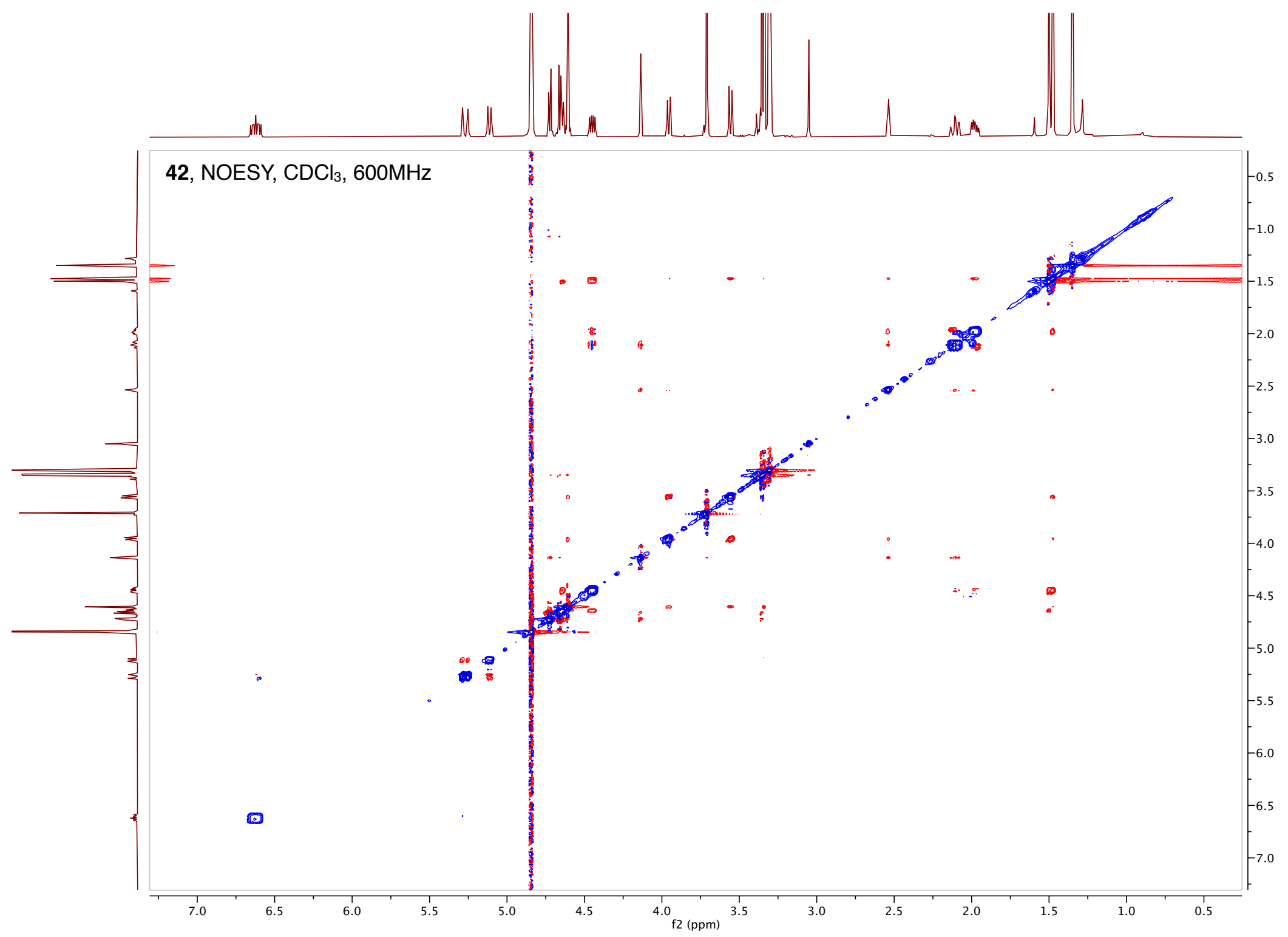




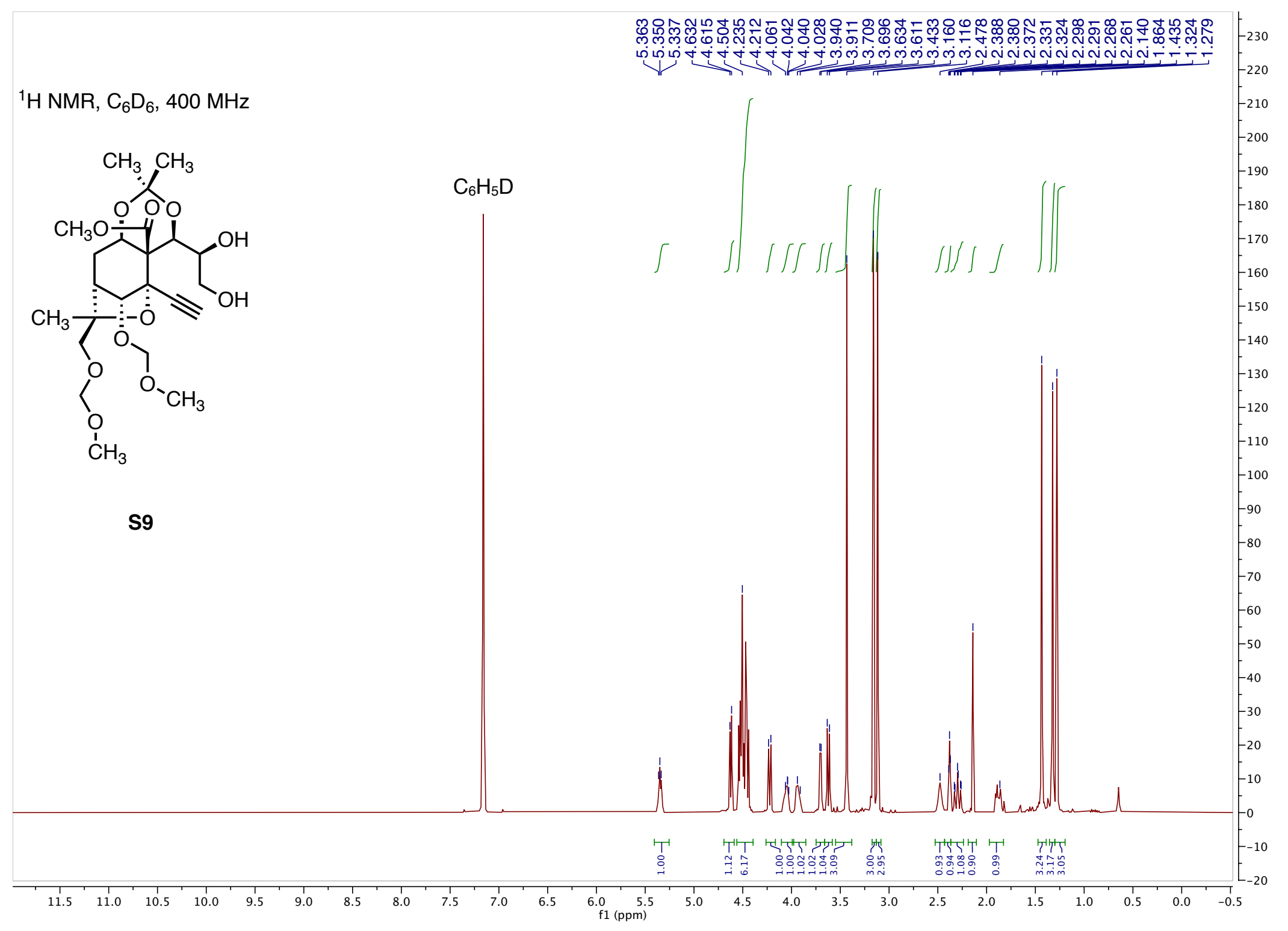




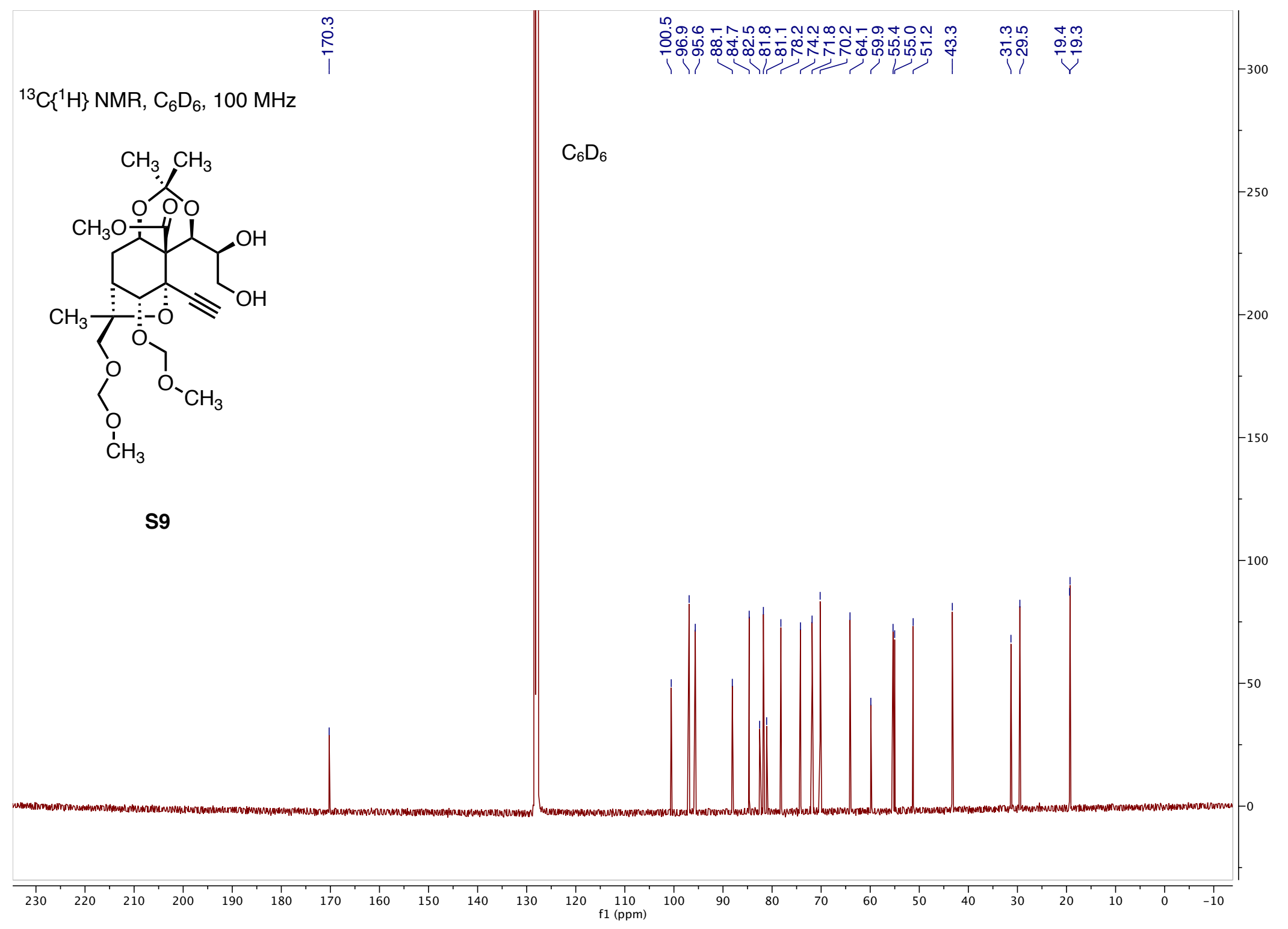




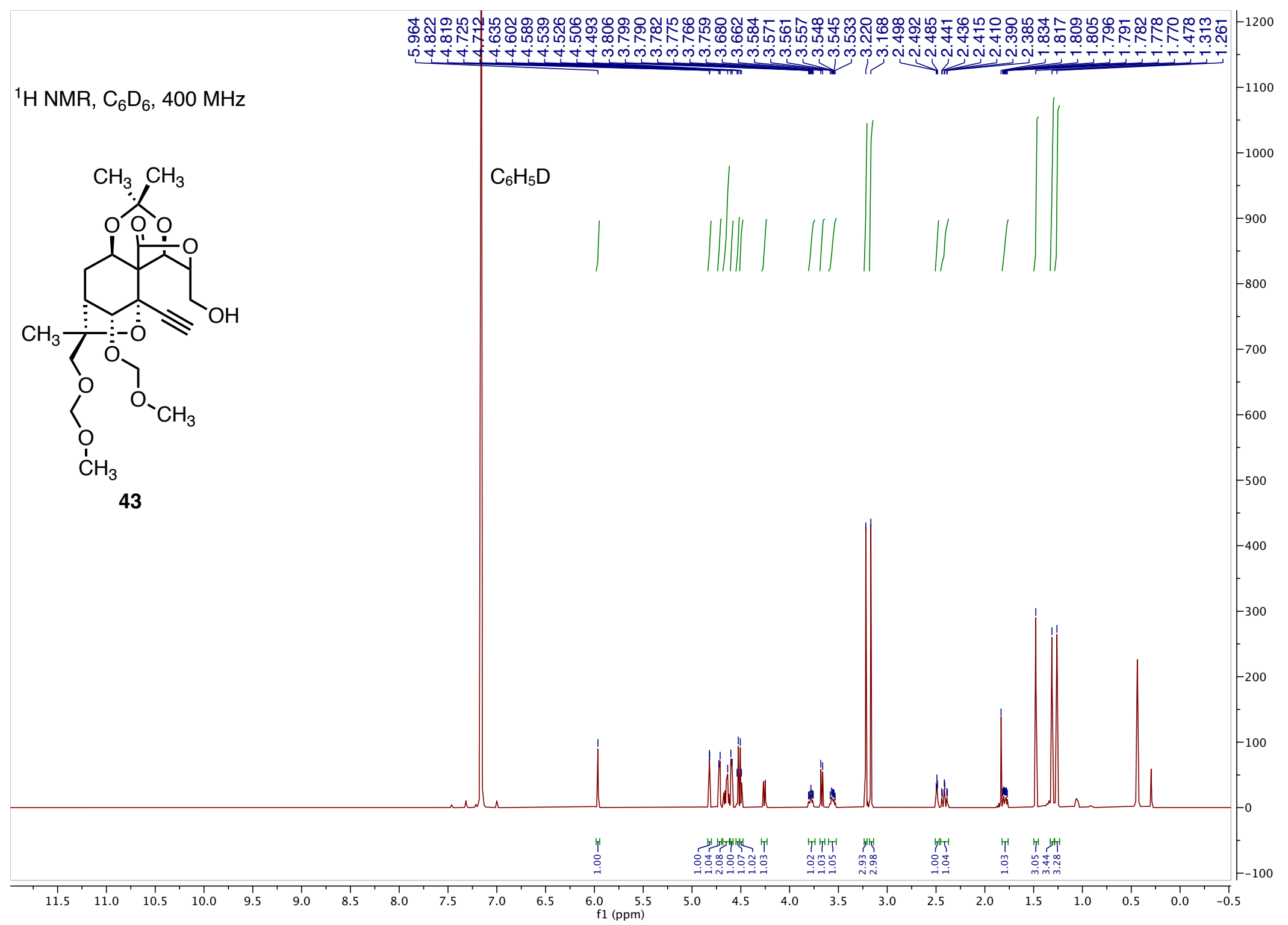




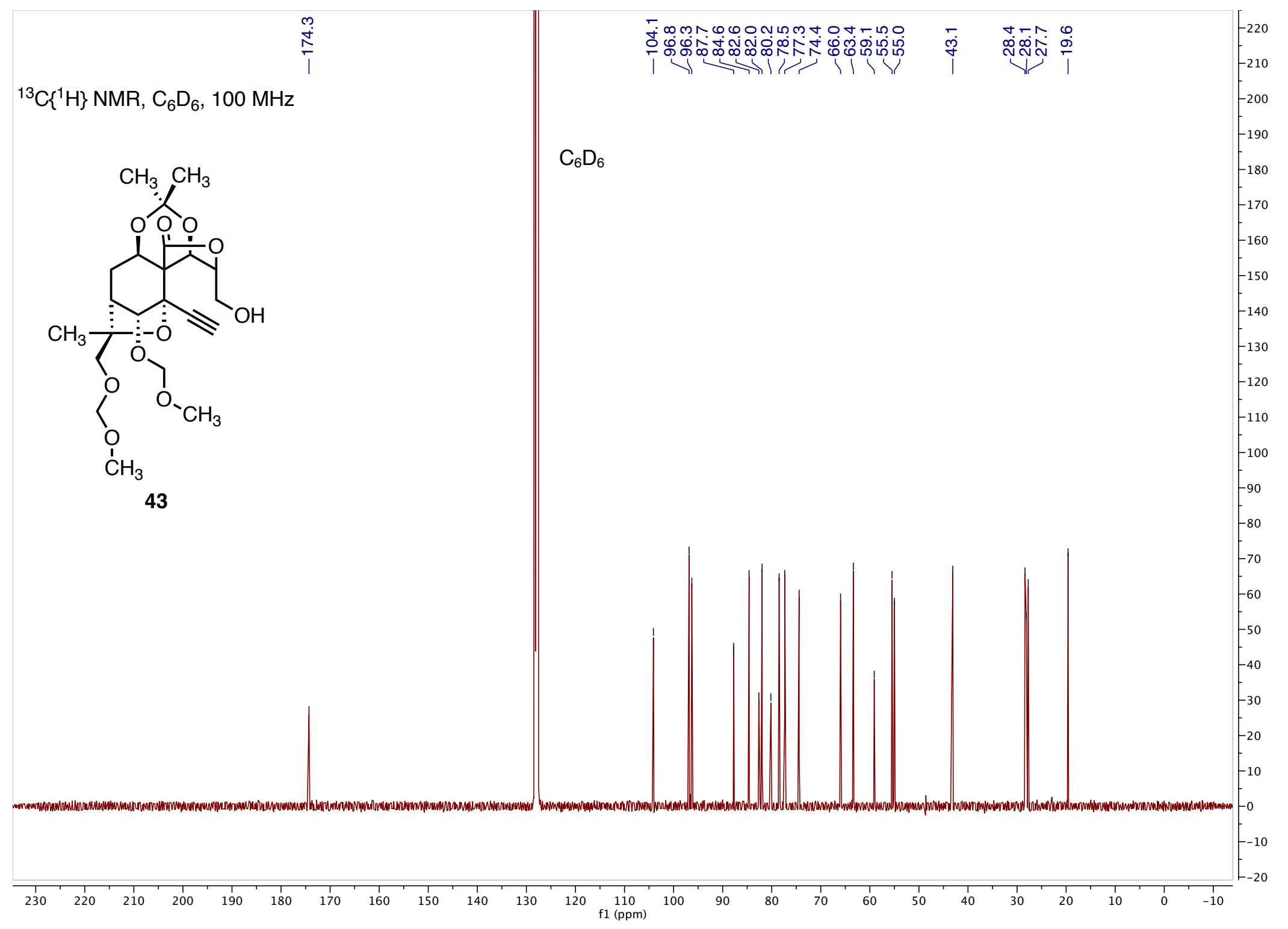




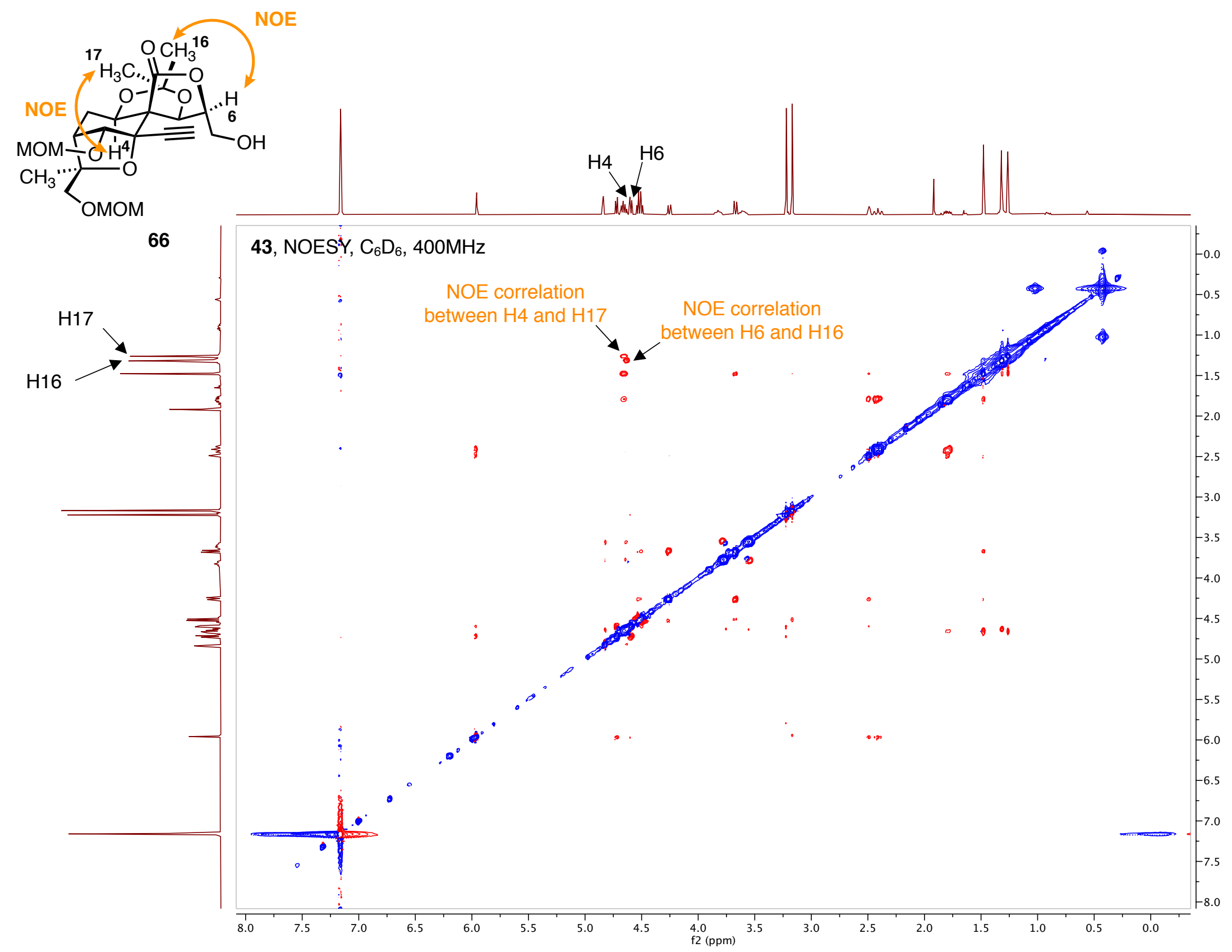




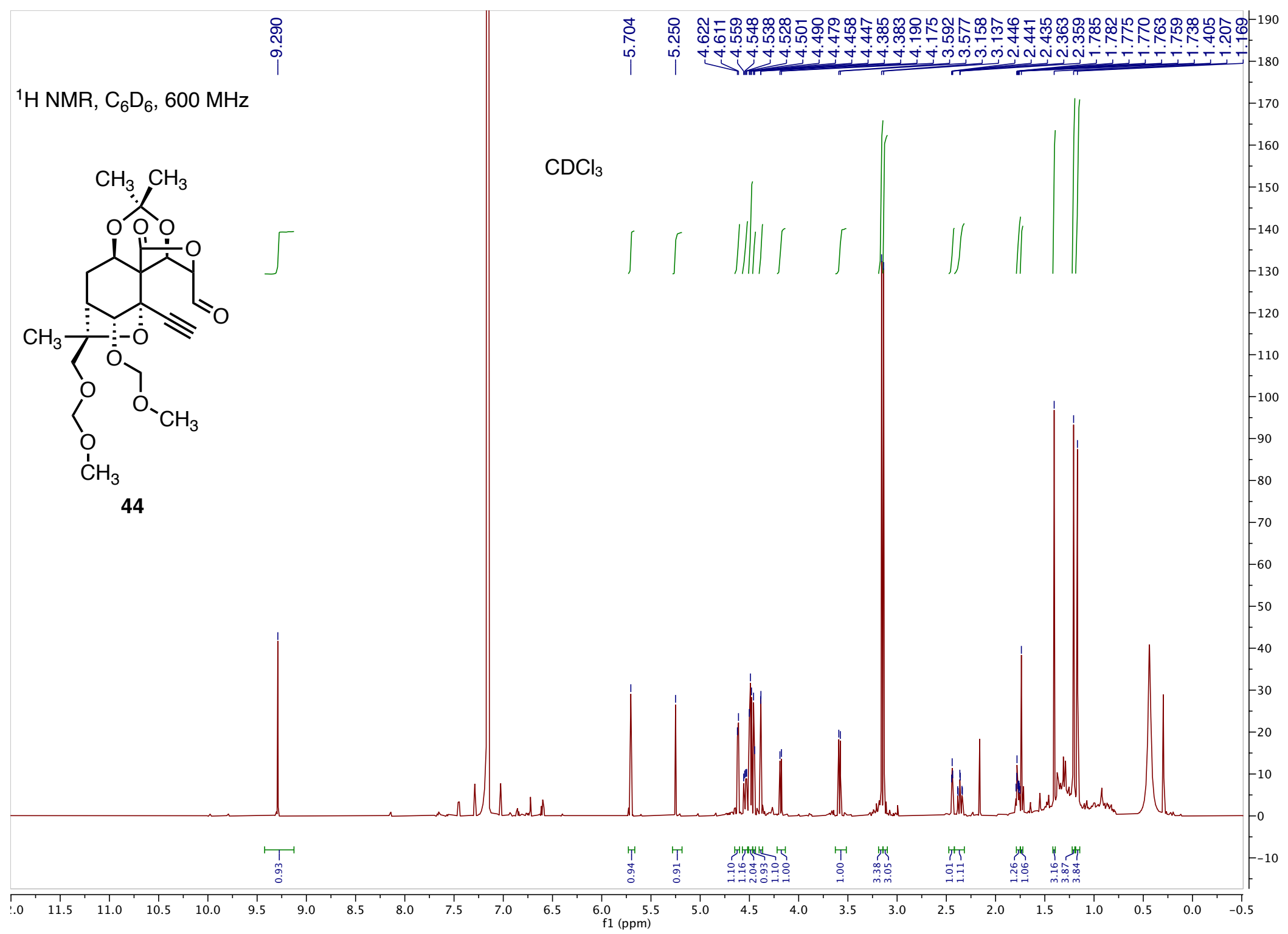




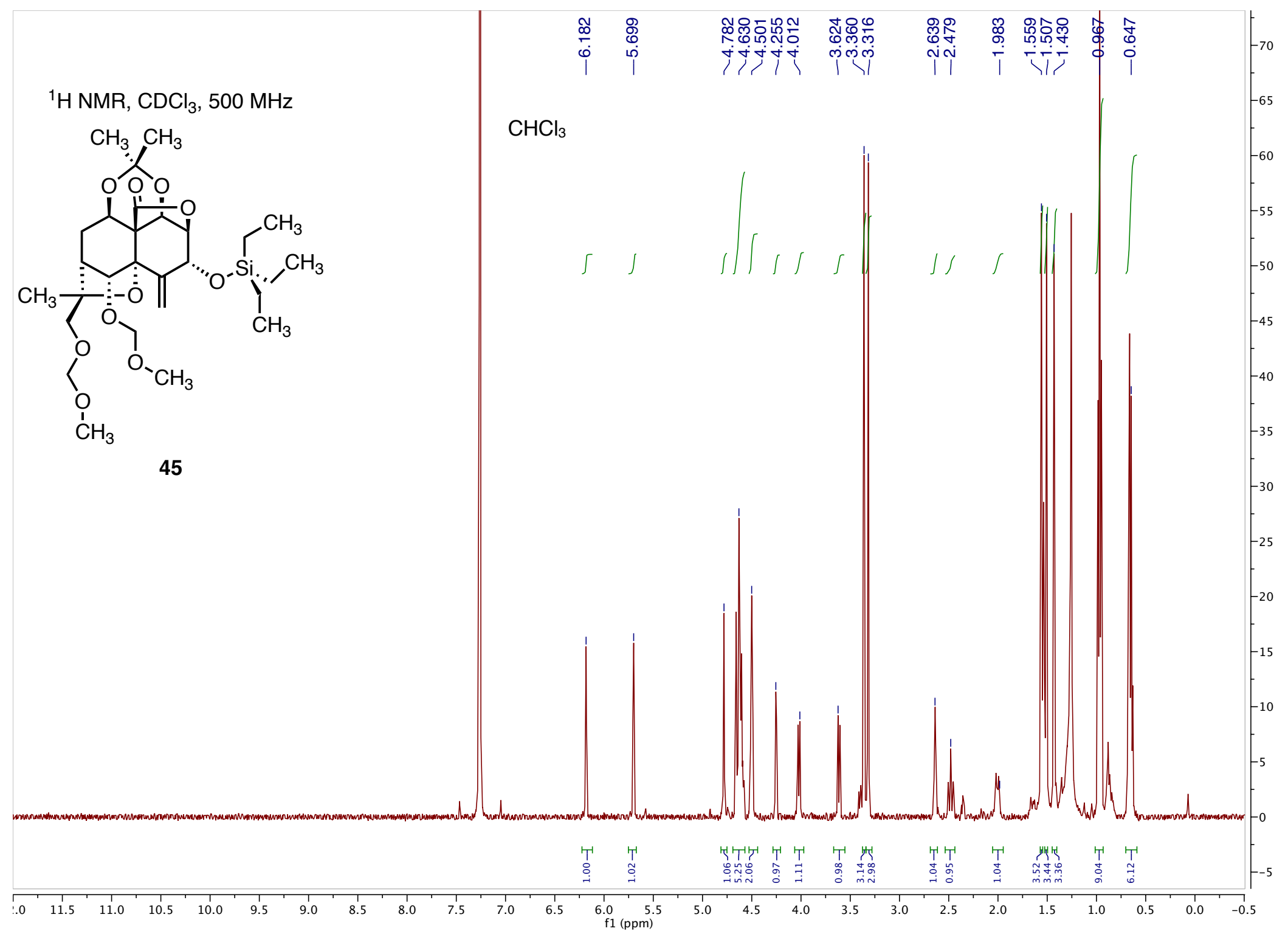




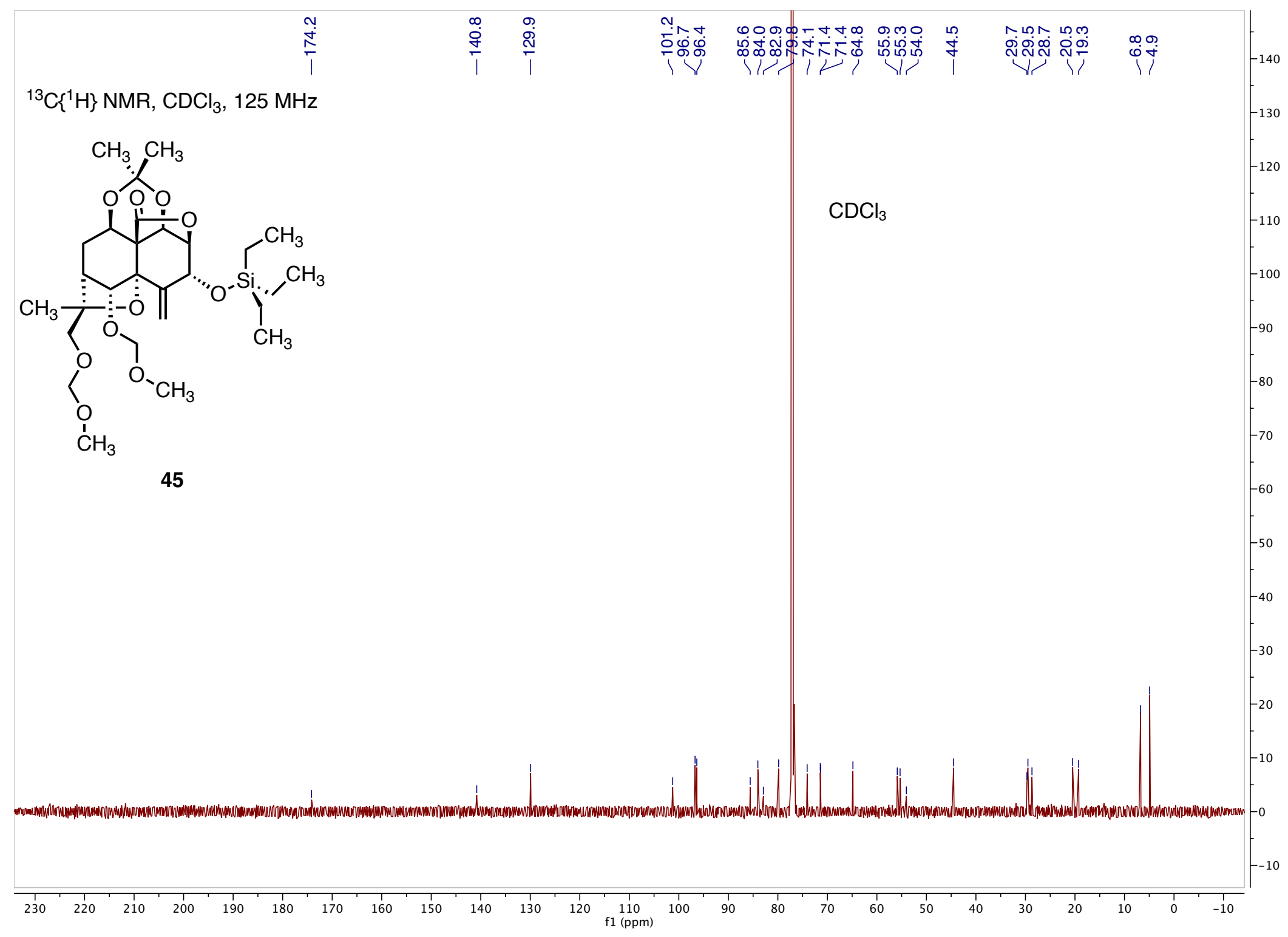




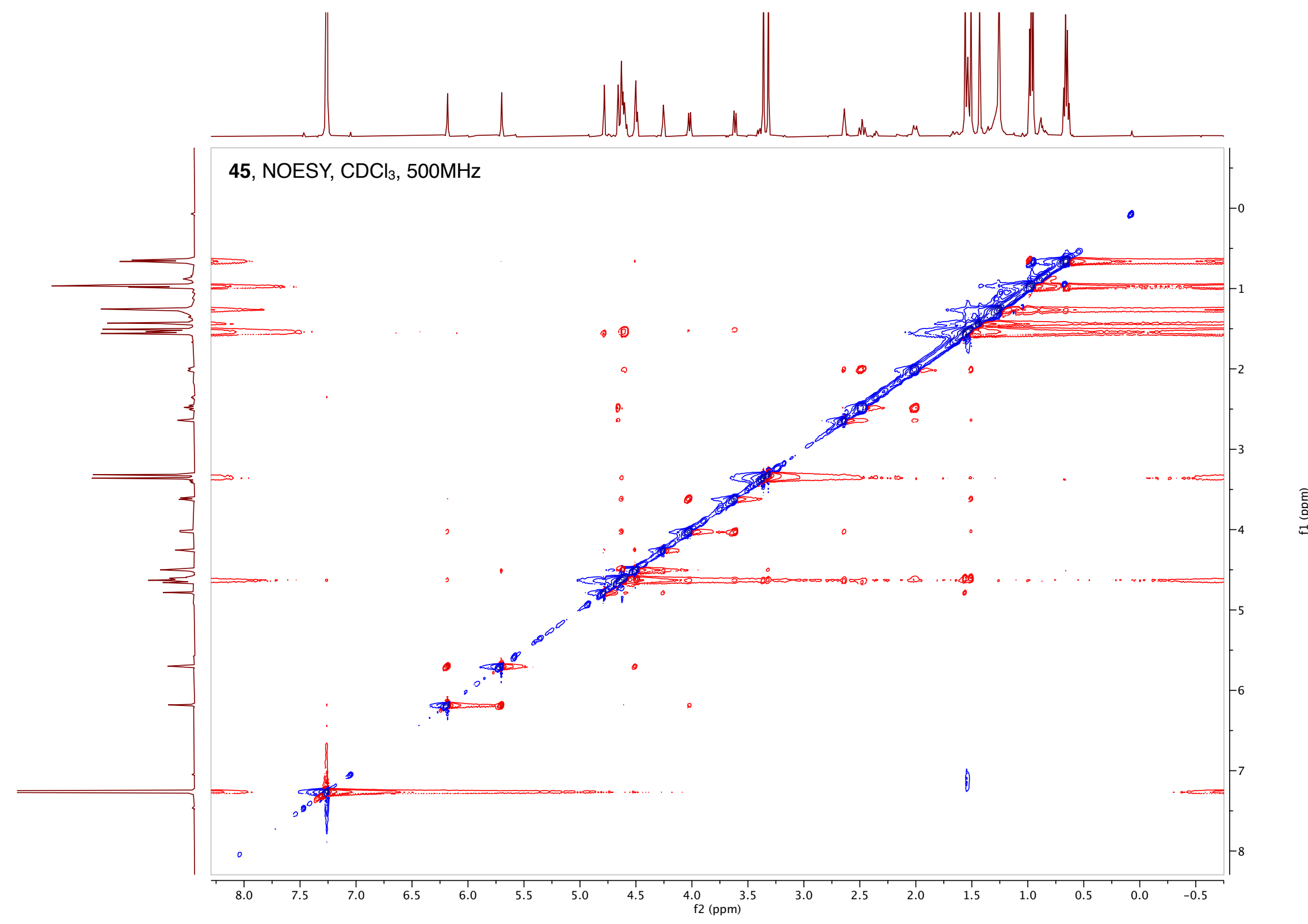




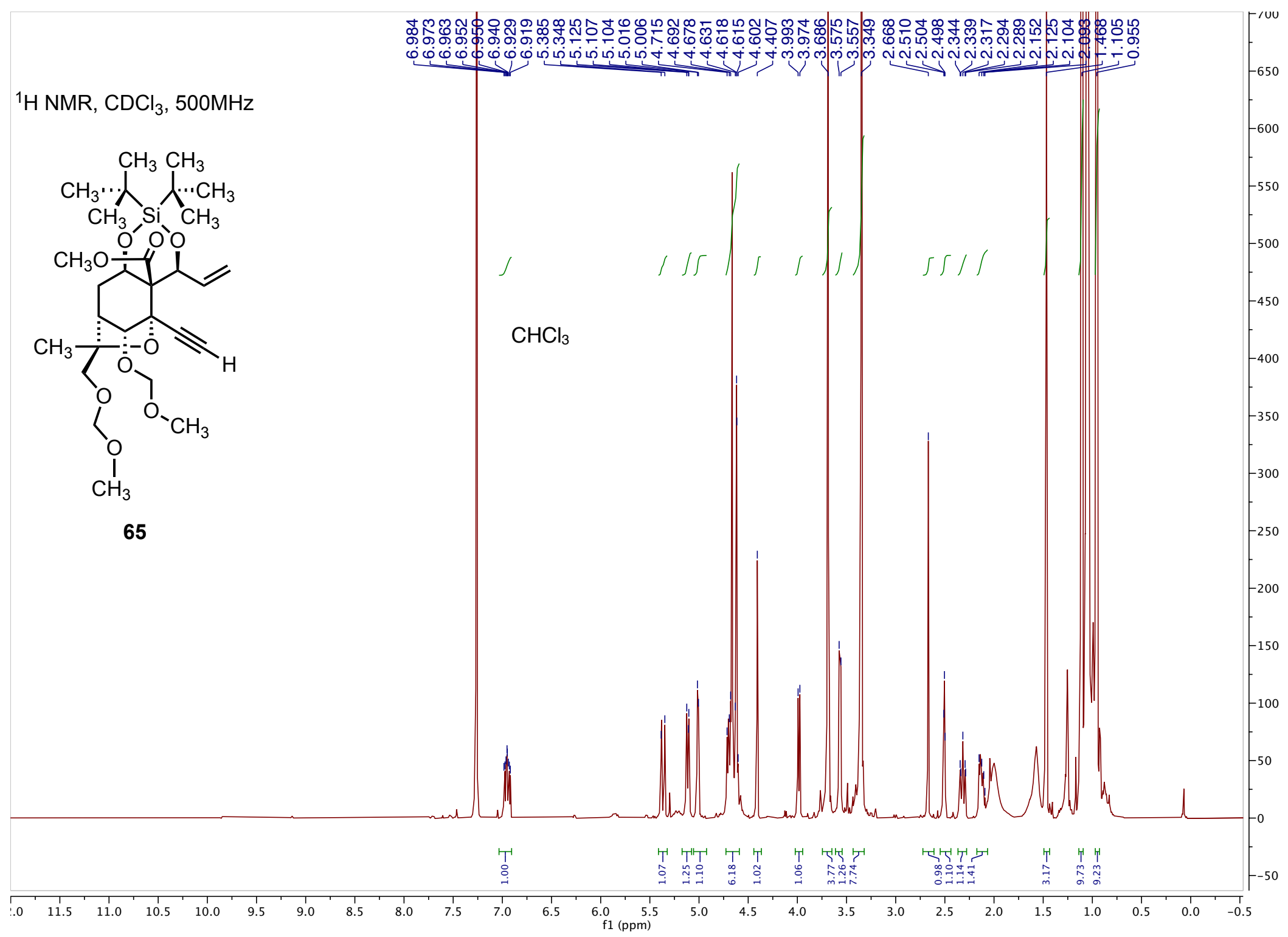




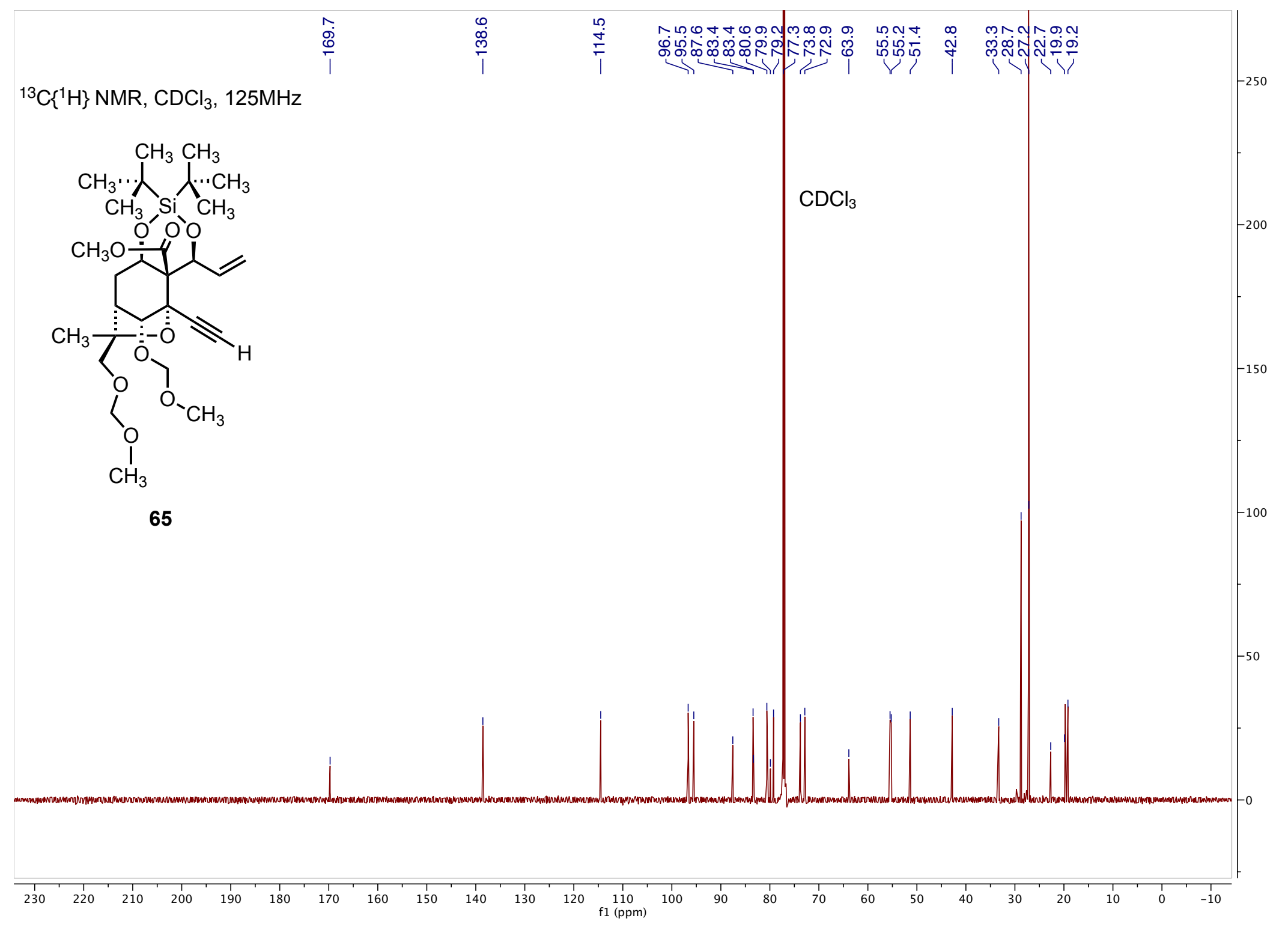




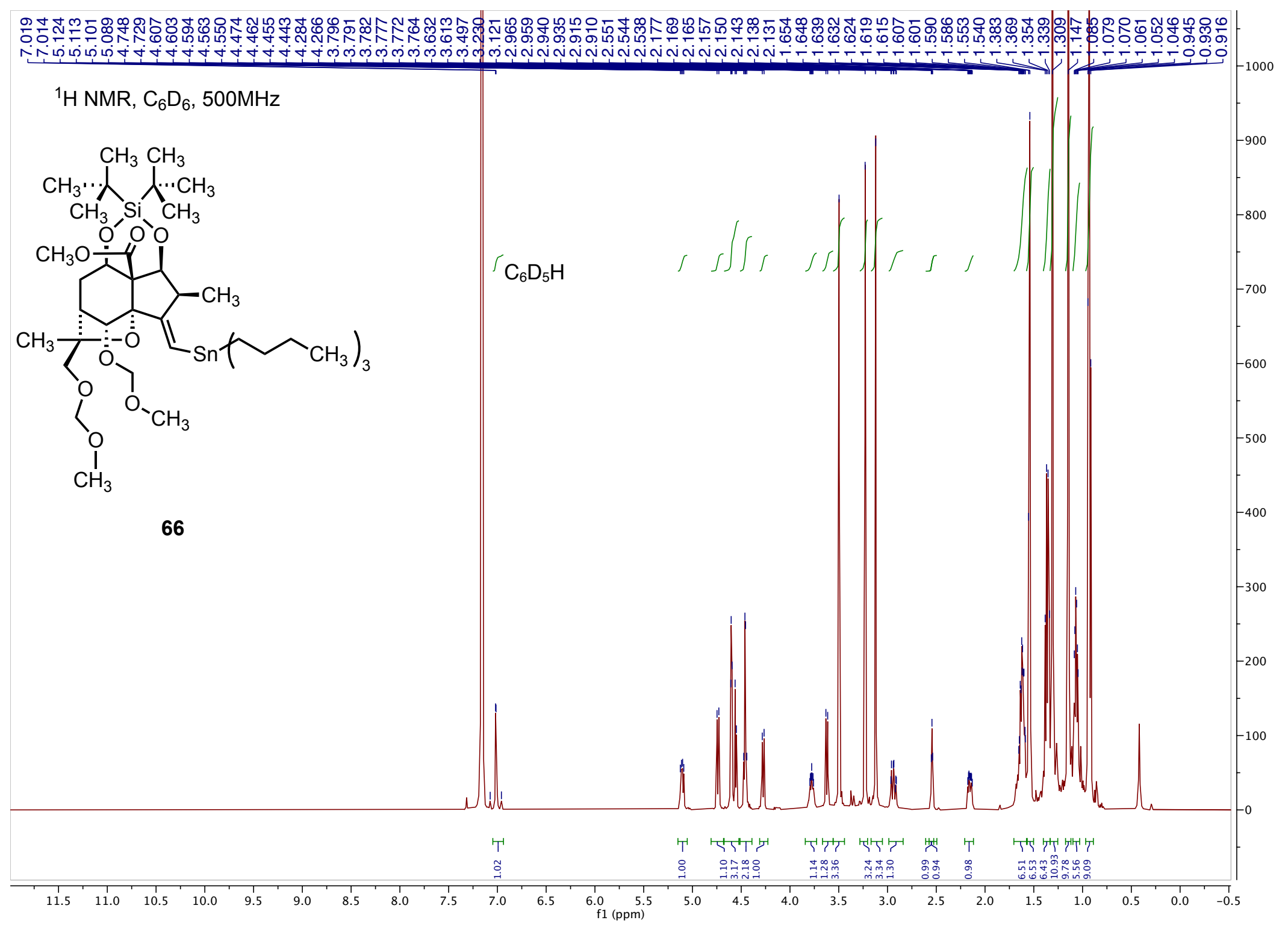




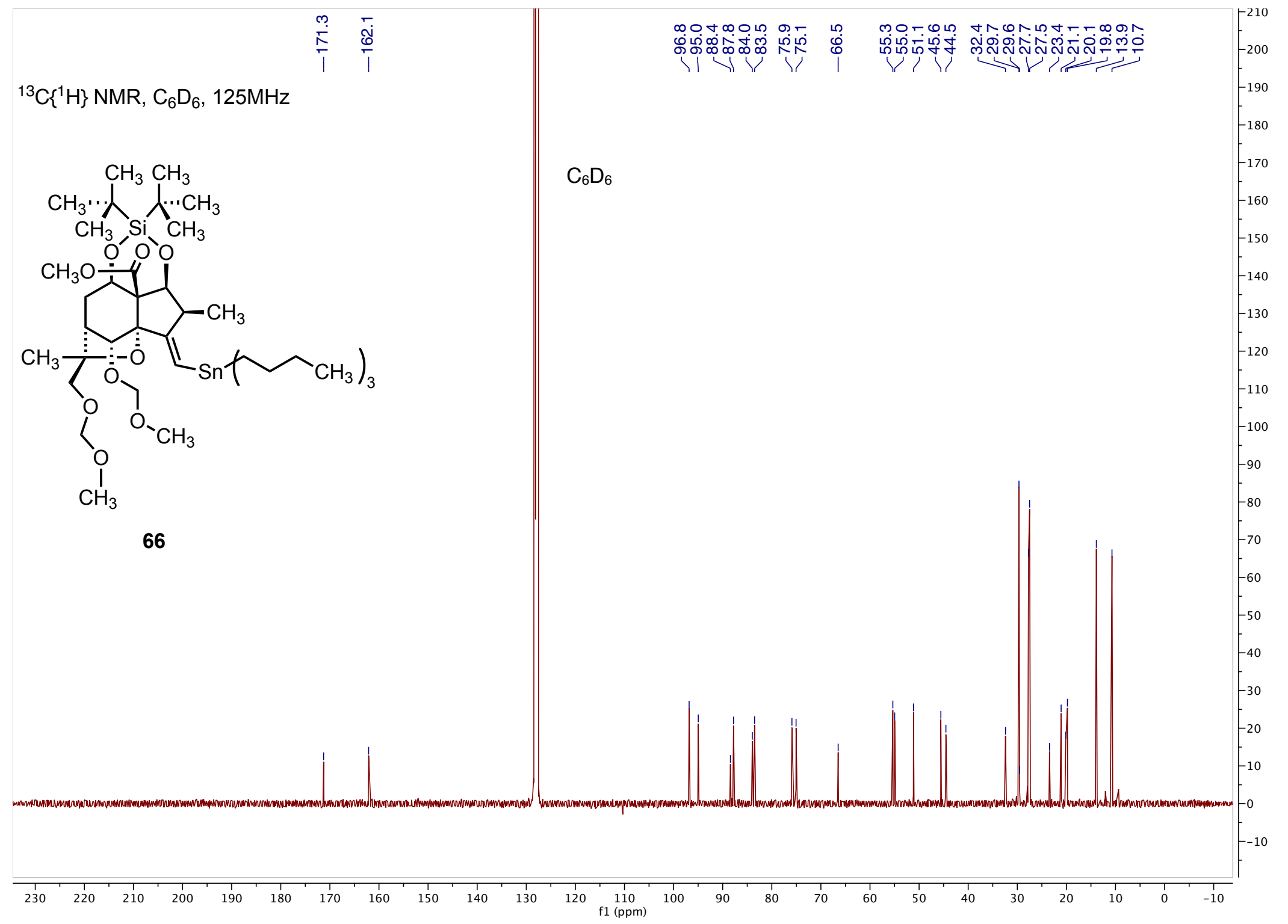




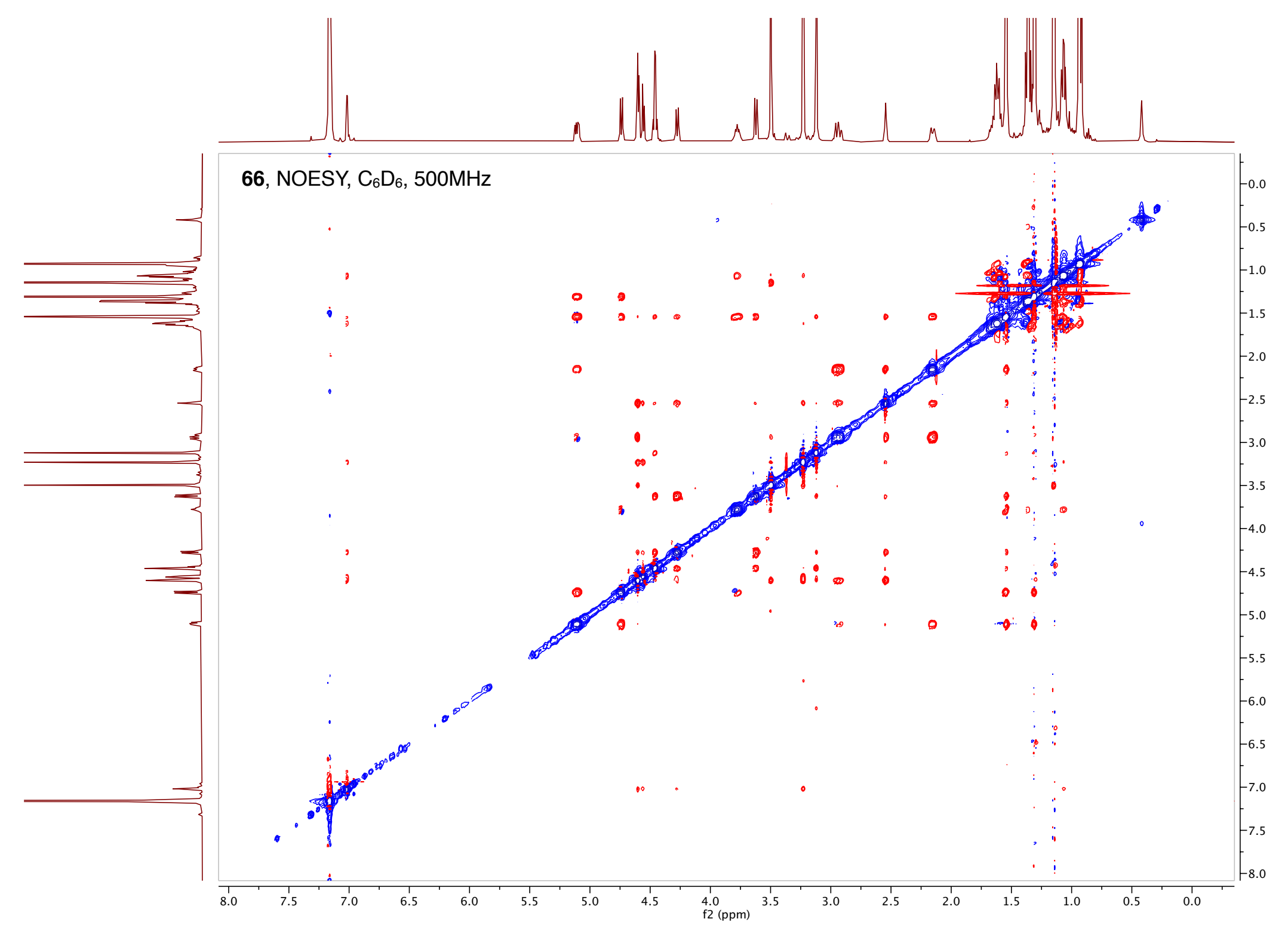




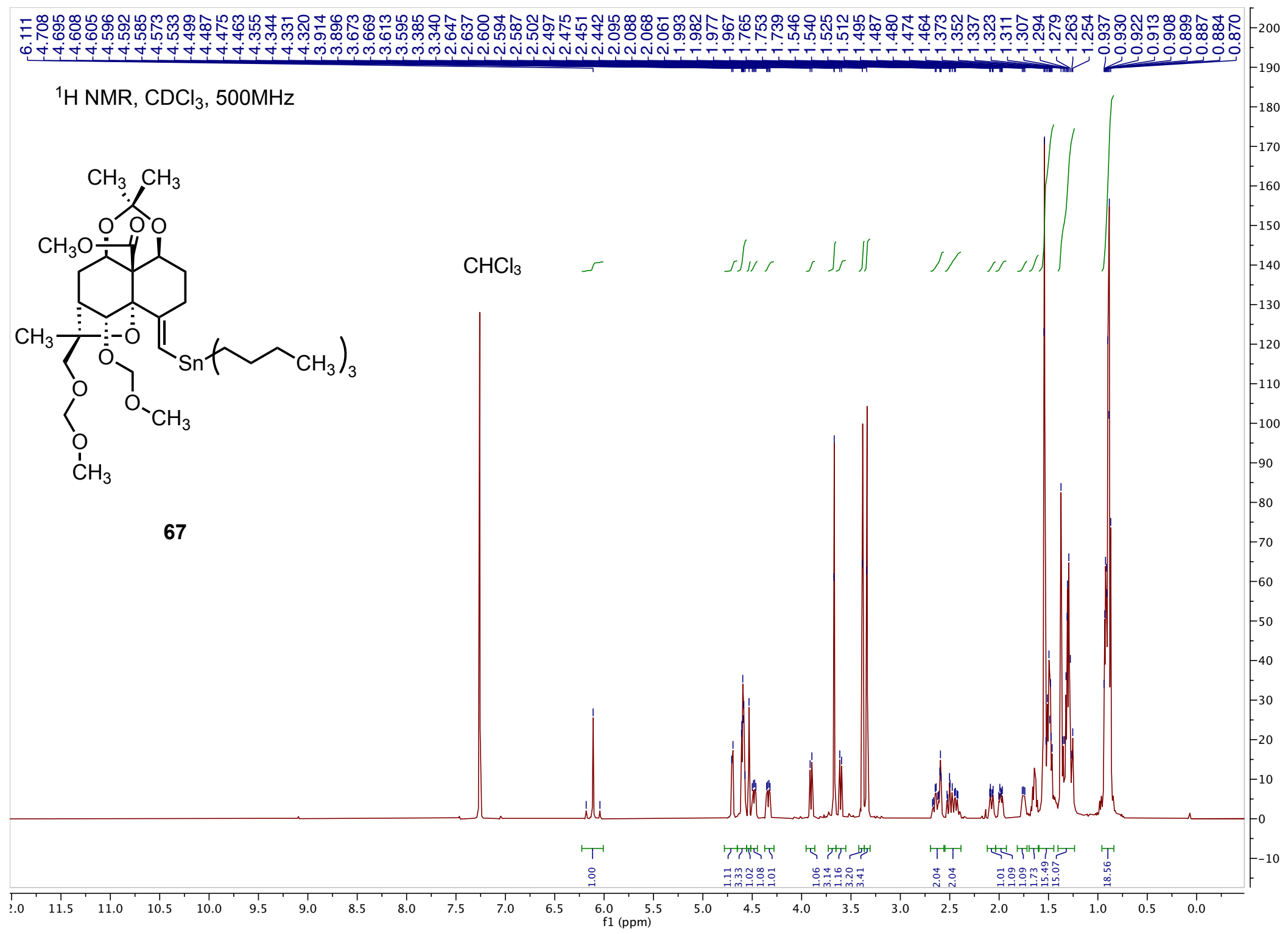




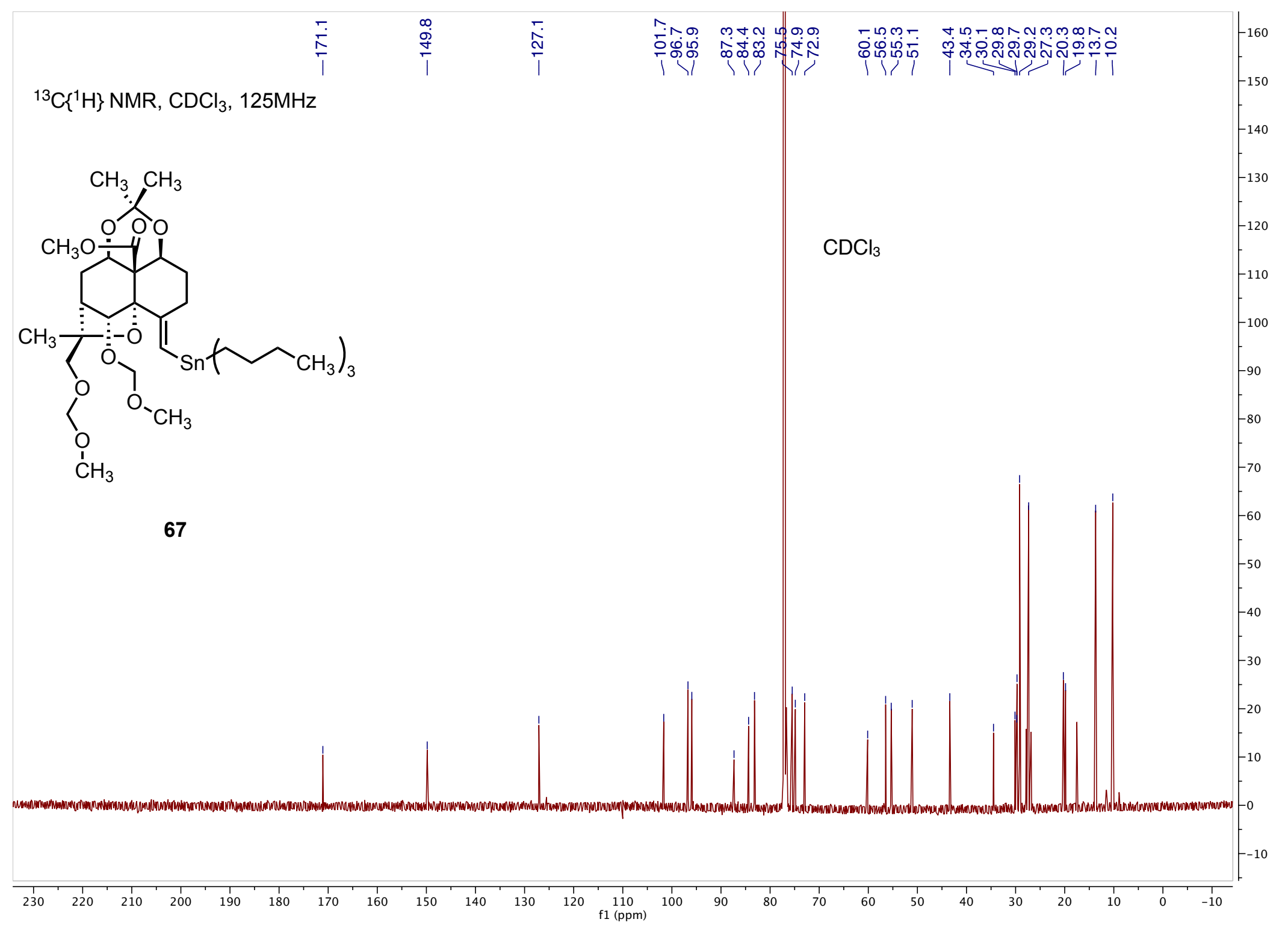




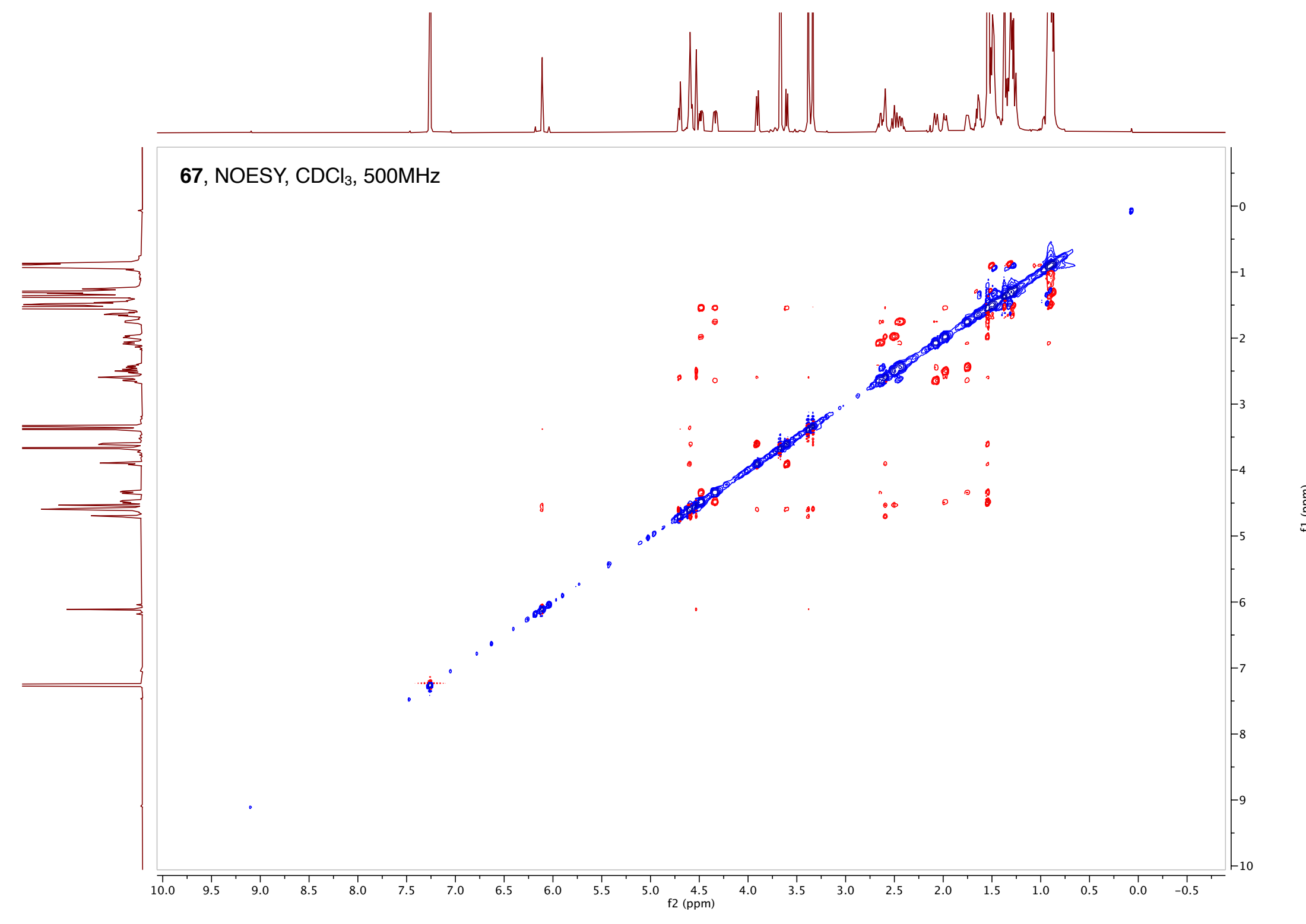




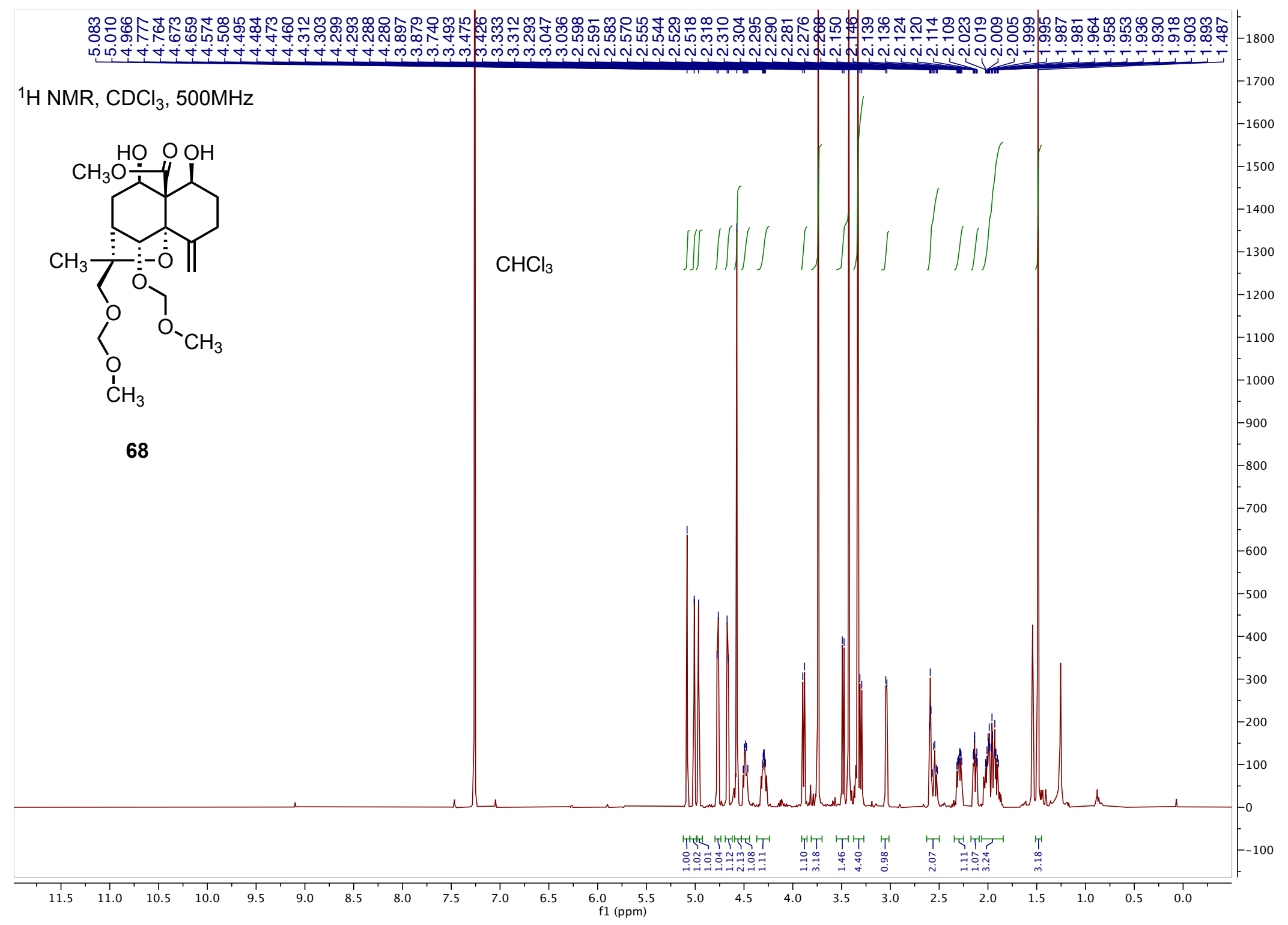




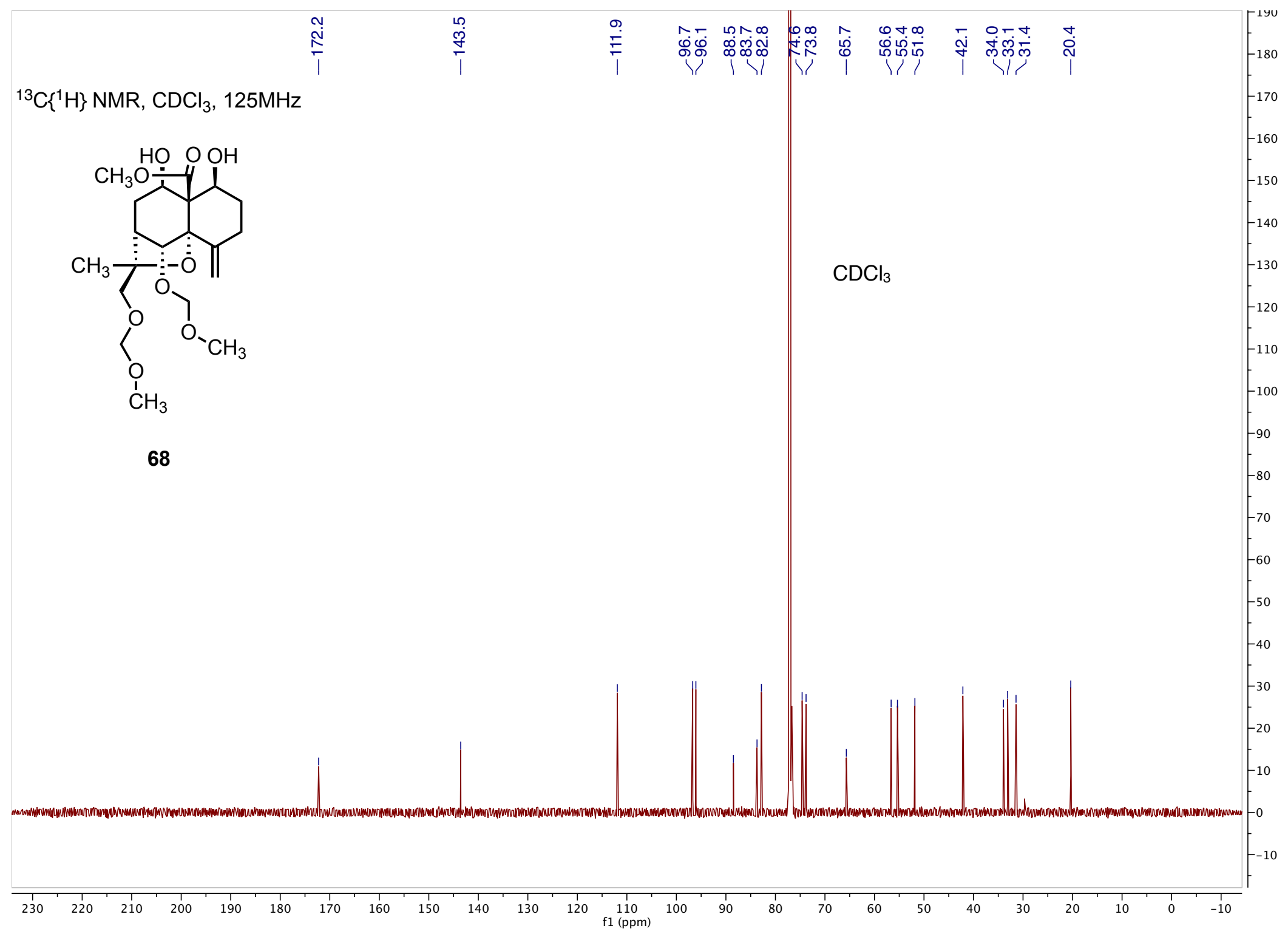




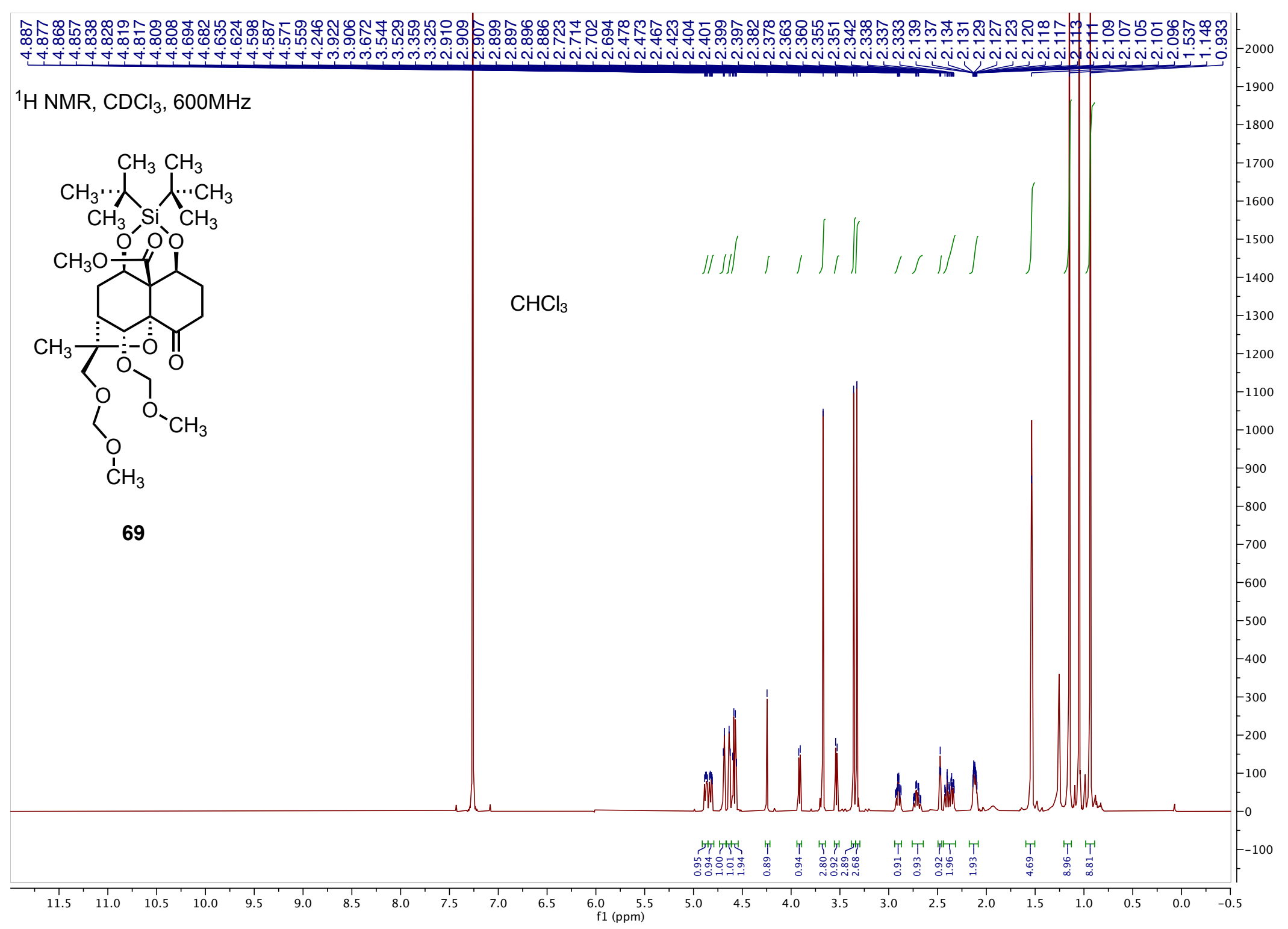




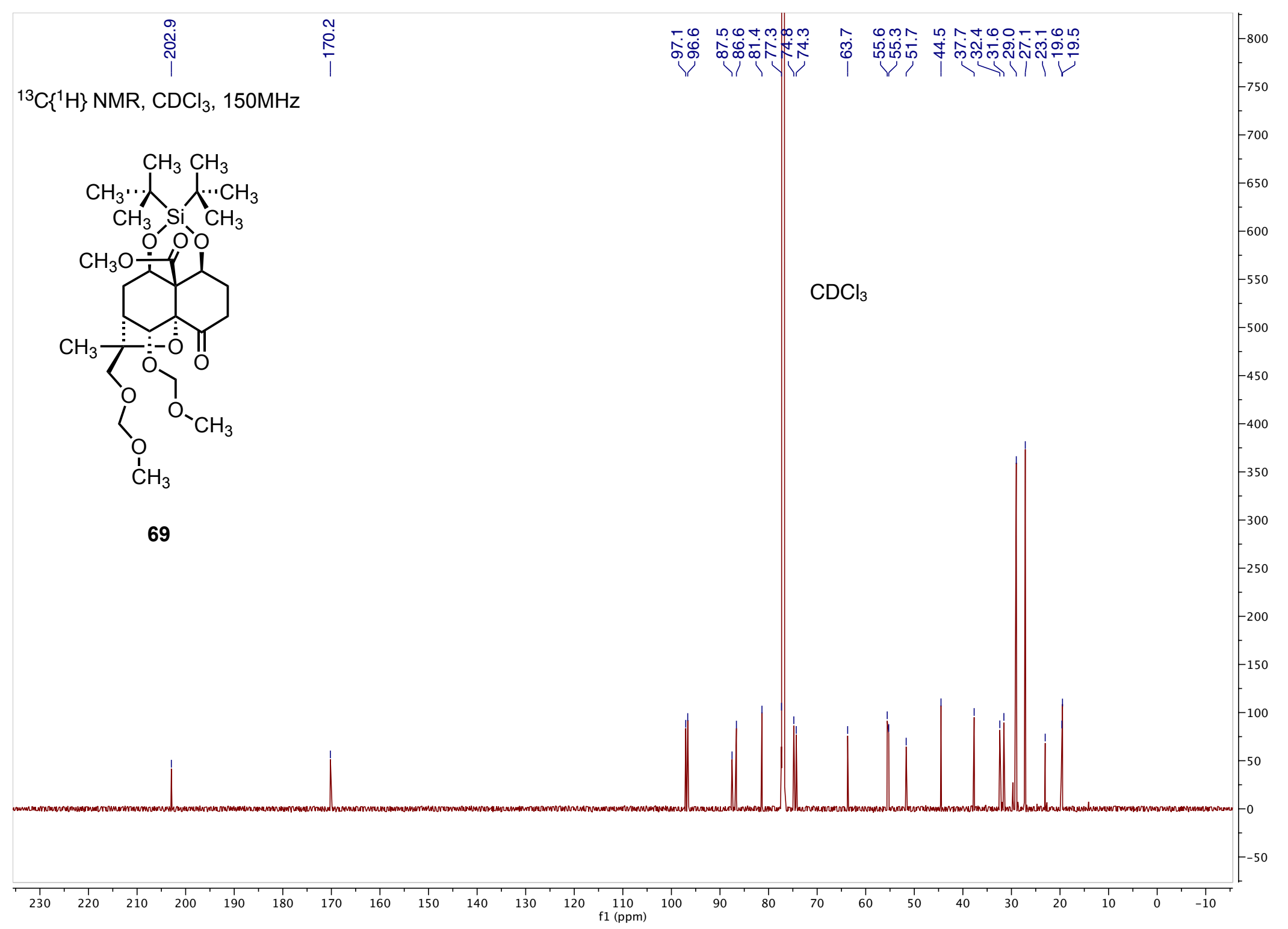




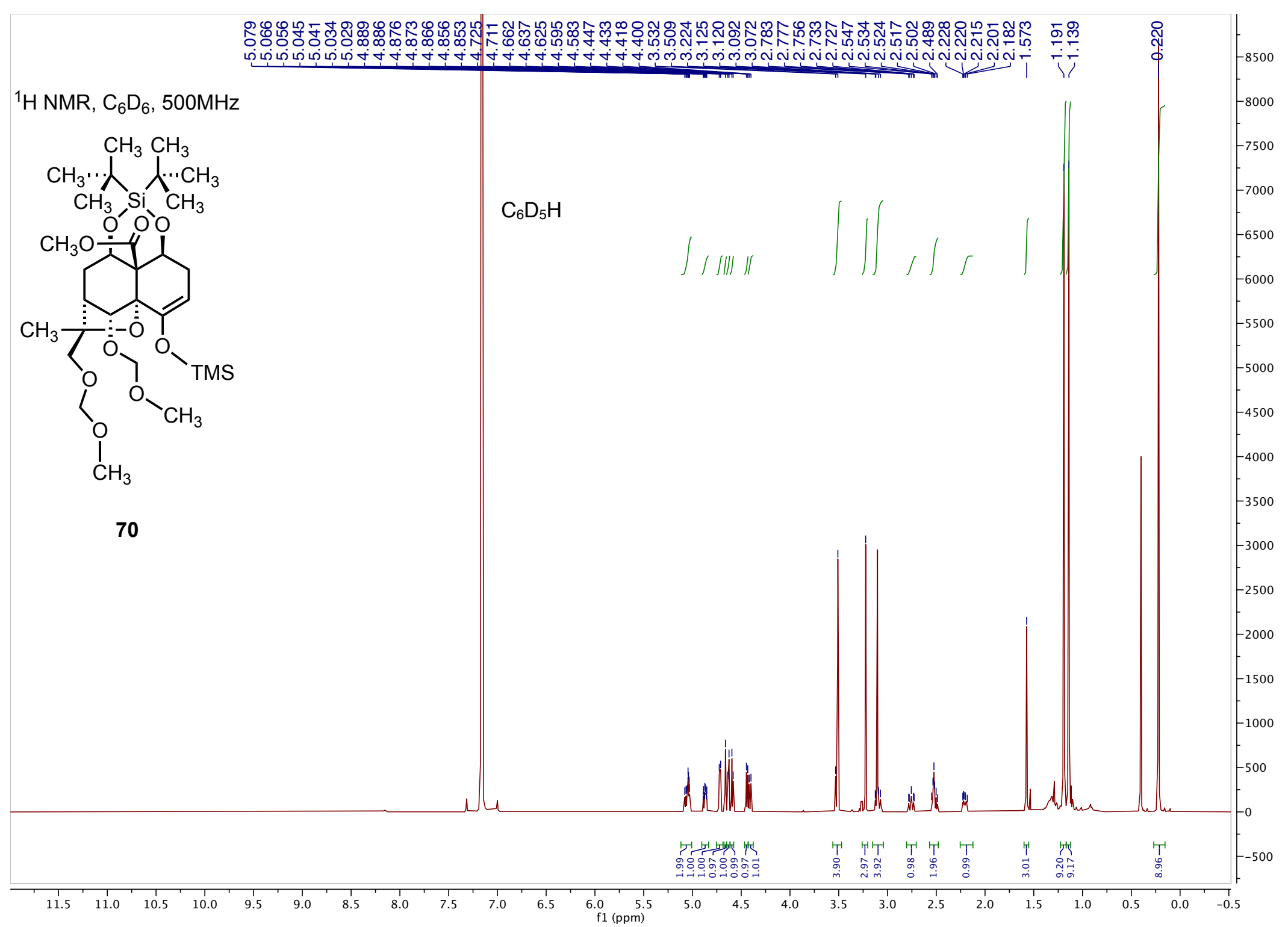




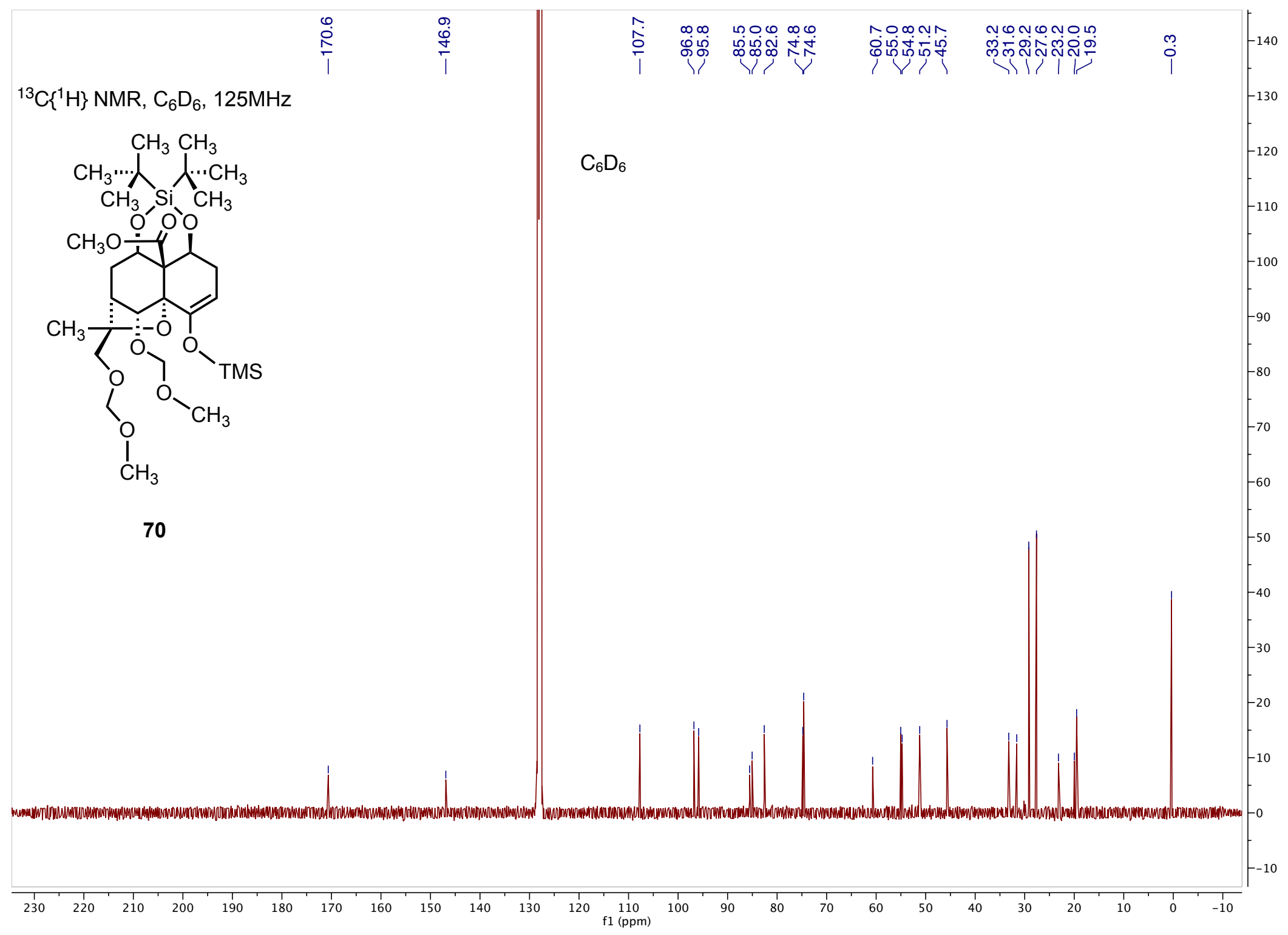




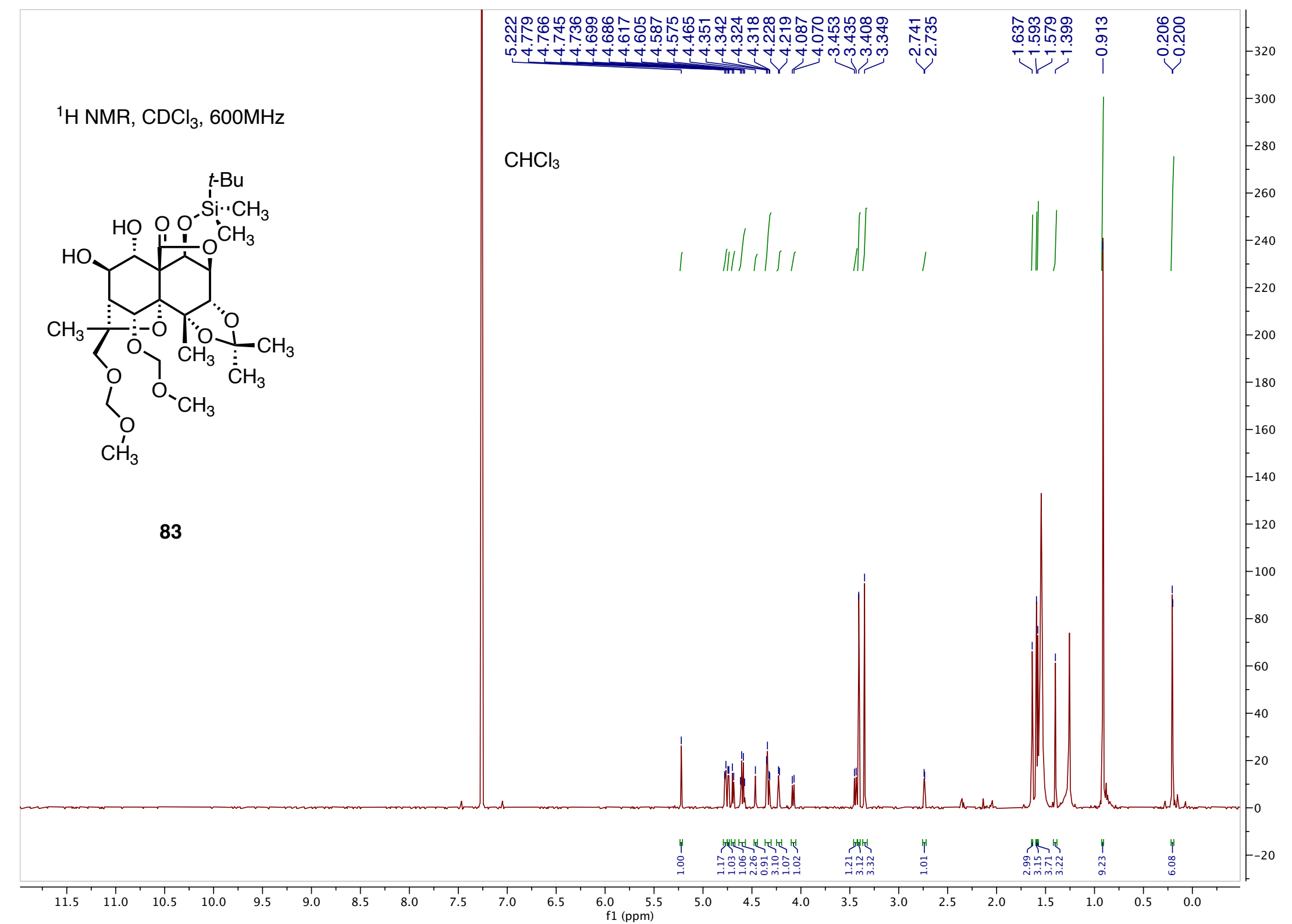




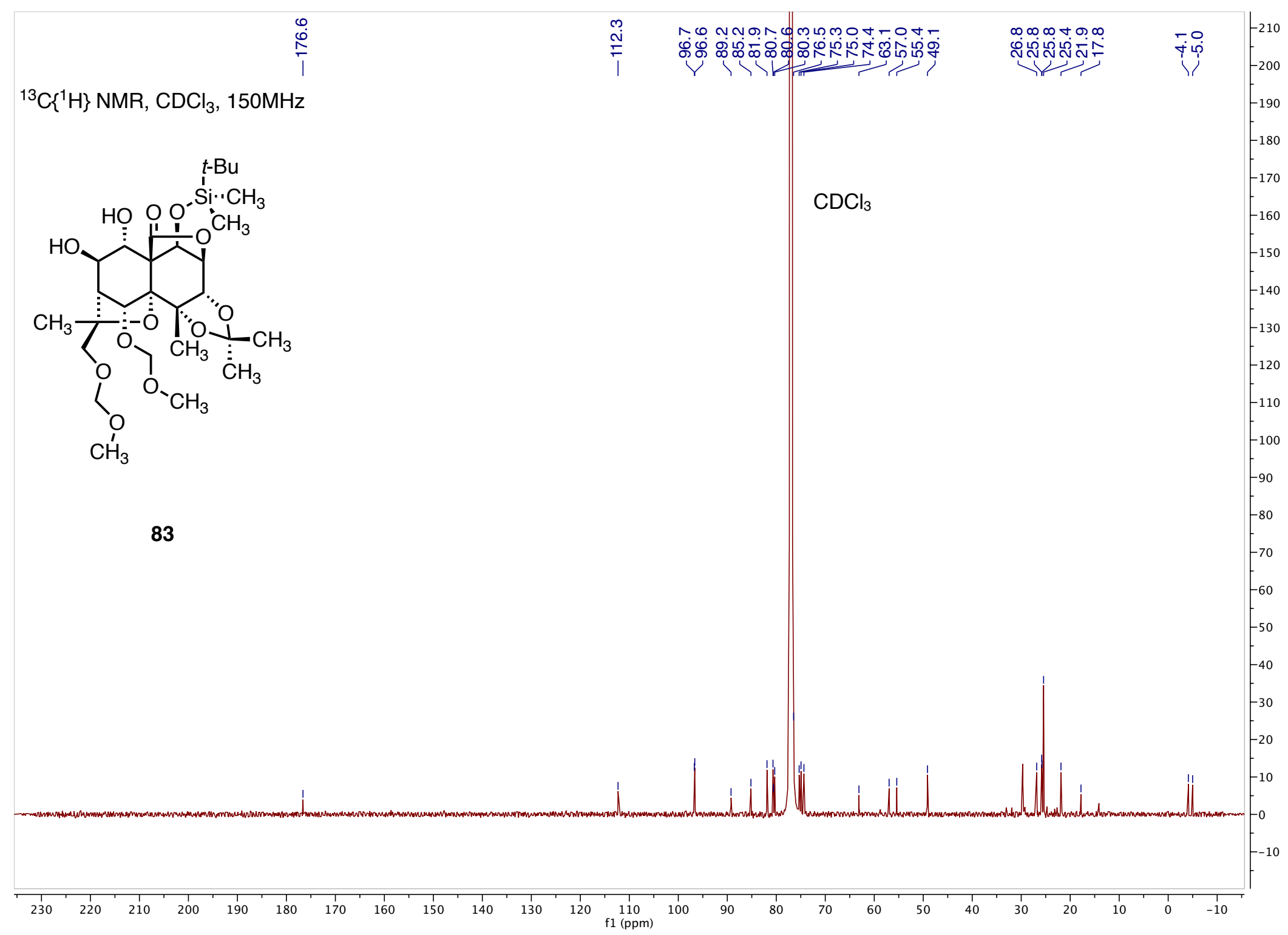




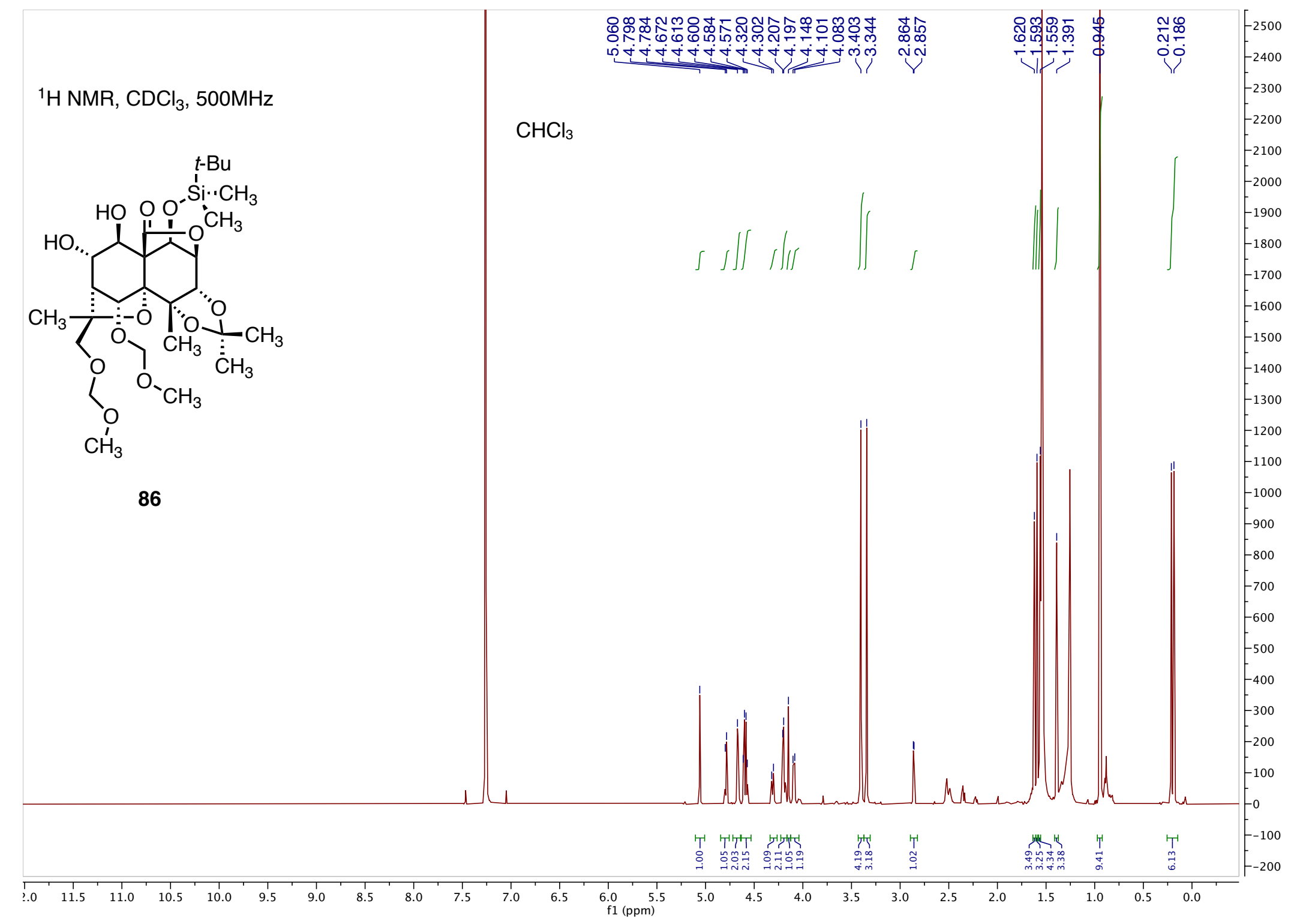




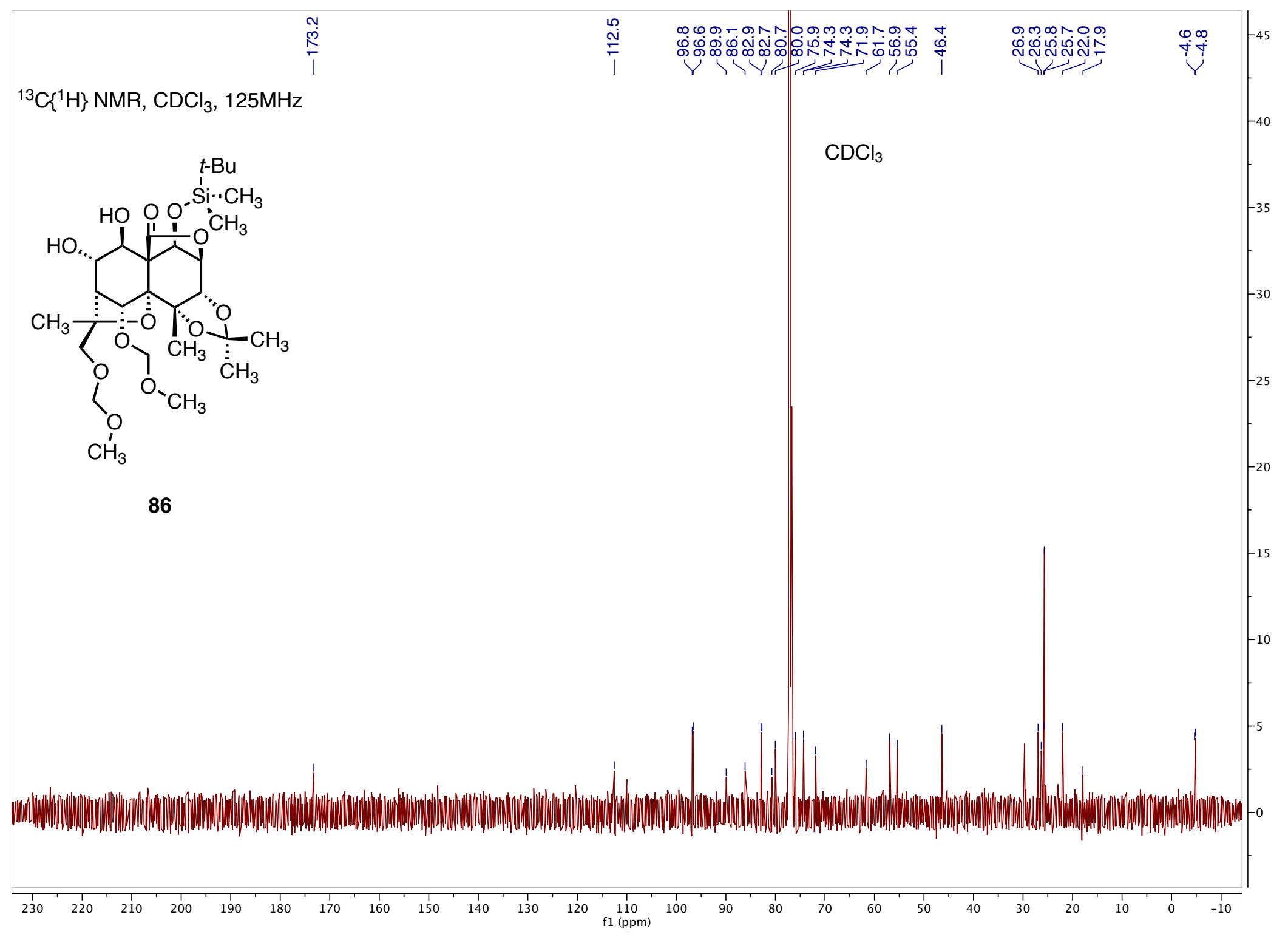




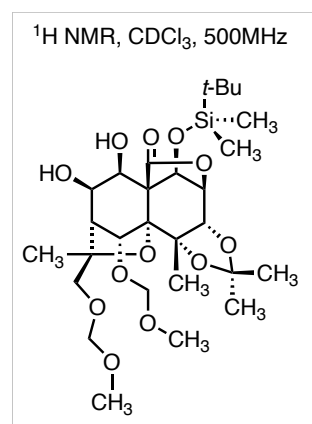

88

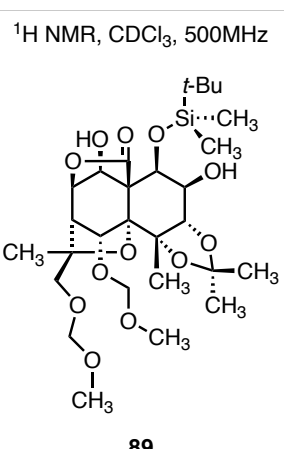

${ }^{1} \mathrm{H} \mathrm{NMR}, \mathrm{CDCl}_{3}, 500 \mathrm{MHz}$

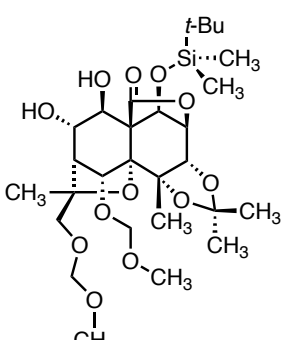

86
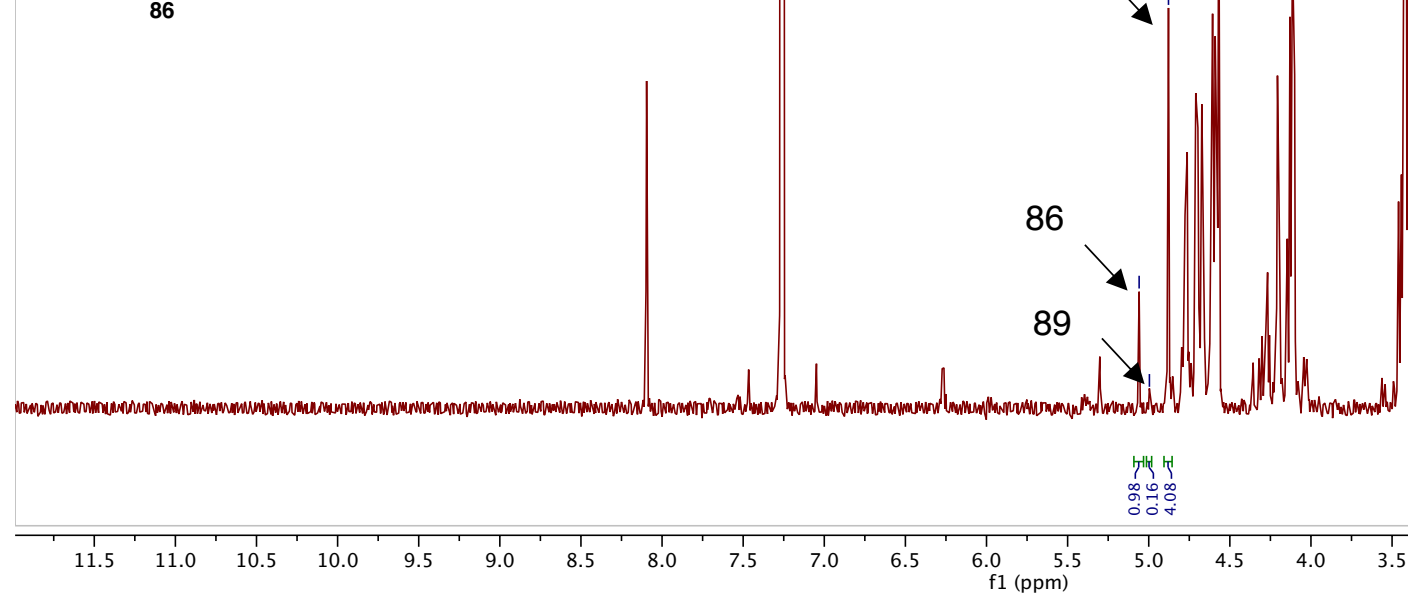

$\mathrm{CHCl}_{3}$

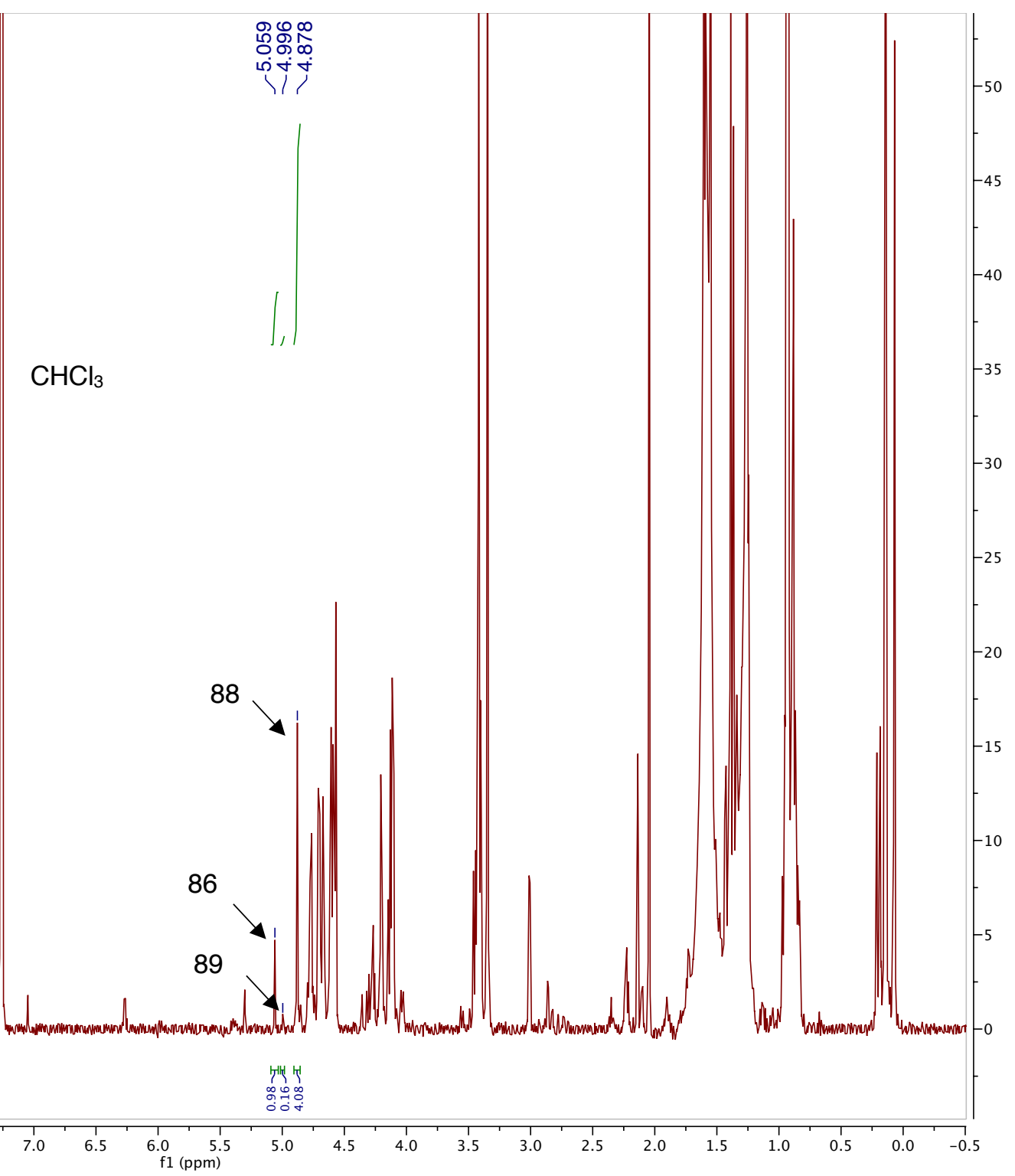




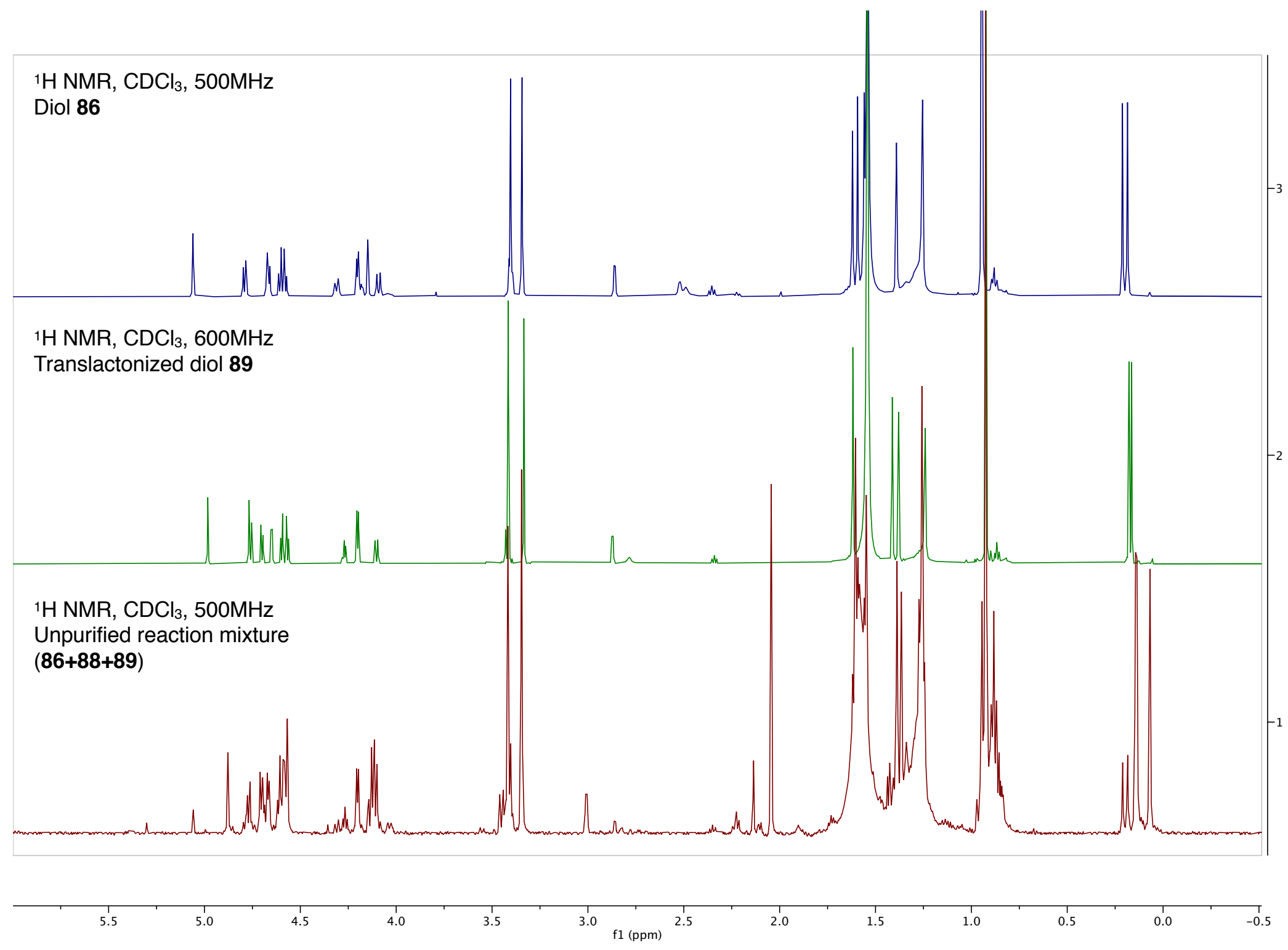




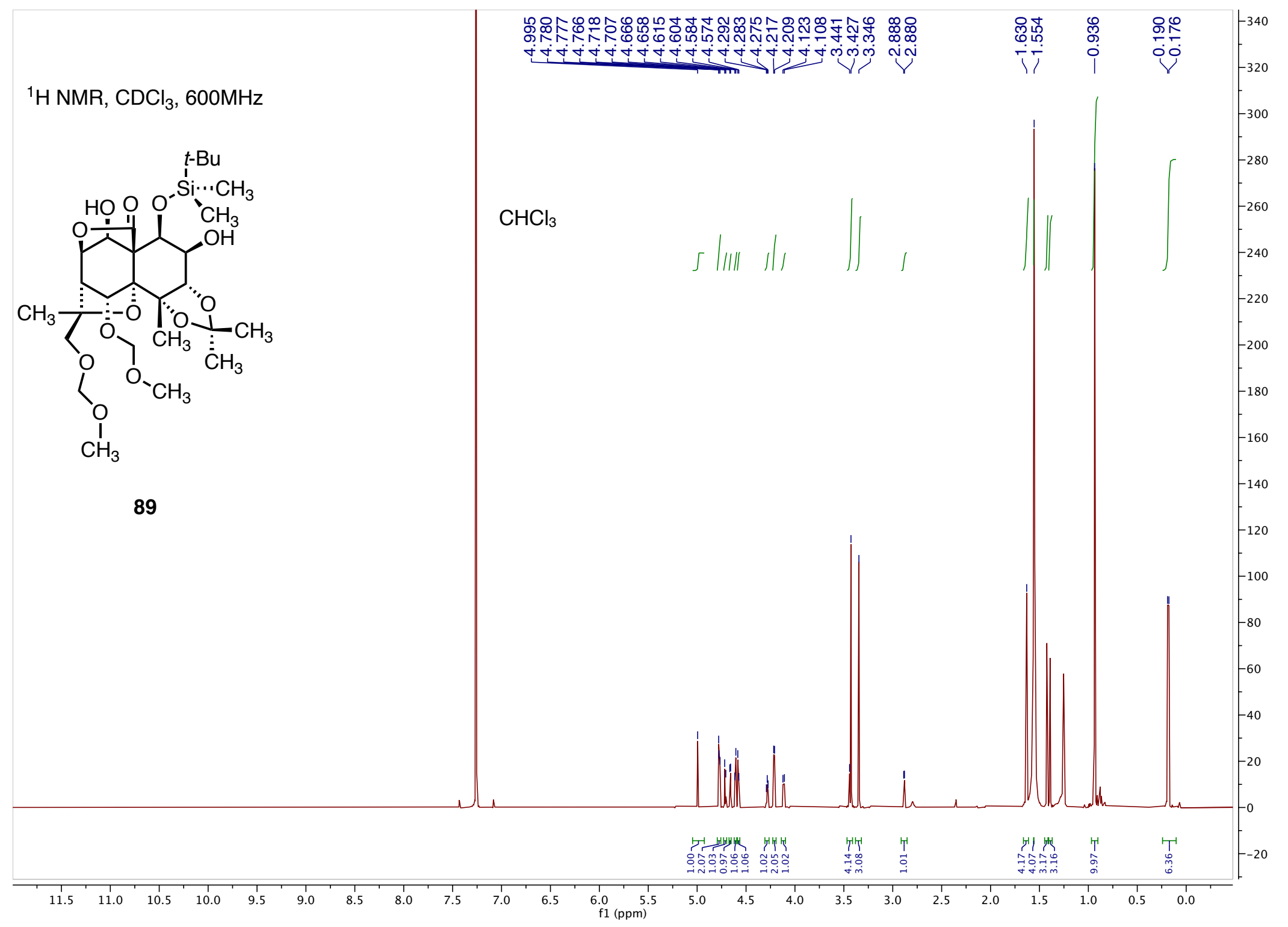




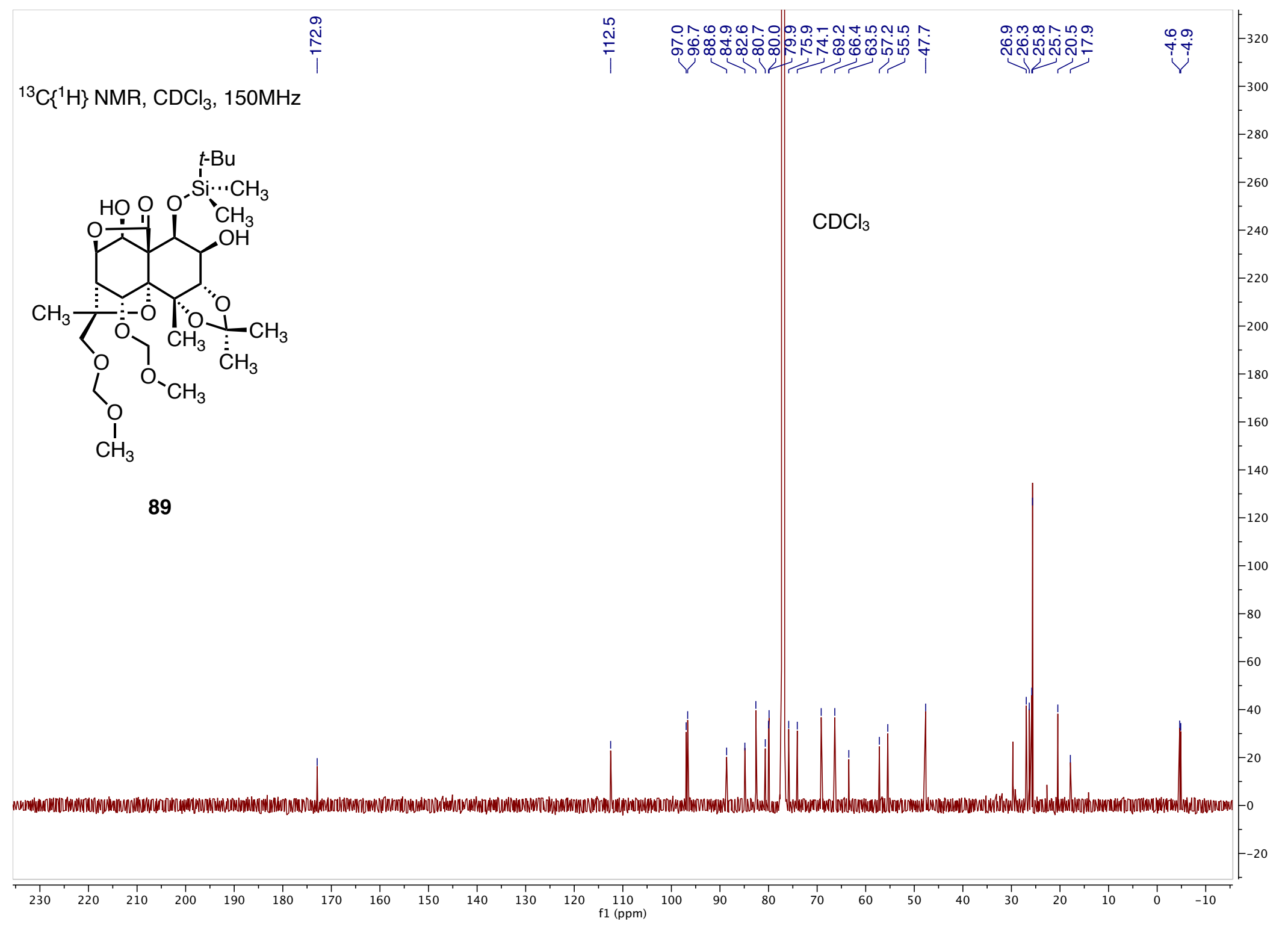




\section{Bibliography.}

(1) White, J. D.; Shin, H.; Kim, T.-S.; Cutshall, N. S. Total Synthesis of the Sesquiterpenoid Polyols ( \pm -Euonyminol and ( \pm -3,4-Dideoxymaytol, Core Constituents of Esters of the Celastraceae. J. Am. Chem. Soc. 1997, 119, 2404.

(2) Tomanik, M.; Xu, Z.; Herzon, S. B. Enantioselective Synthesys of Euonyminol. J. Am. Chem. Soc. 2021, 143, 699.

(3) Frisch, M. J.; Trucks, G. W.; Schlegel, H. B.; Scuseria, G. E.; Robb, M. A.; Cheeseman, J. R.; Scalmani, G.; Barone, V.; Petersson, G. A.; Nakatsuji, H.; Li, X.; Caricato, M.; Marenich, A. V.; Bloino, J.; Janesko, B. G.; Gomperts, R.; Mennucci, B.; Hratchian, H. P.; Ortiz, J. V.; Izmaylov, A. F.; Sonnenberg, J. L.; Williams; Ding, F.; Lipparini, F.; Egidi, F.; Goings, J.; Peng, B.; Petrone, A.; Henderson, T.; Ranasinghe, D.; Zakrzewski, V. G.; Gao, J.; Rega, N.; Zheng, G.; Liang, W.; Hada, M.; Ehara, M.; Toyota, K.; Fukuda, R.; Hasegawa, J.; Ishida, M.; Nakajima, T.; Honda, Y.; Kitao, O.; Nakai, H.; Vreven, T.; Throssell, K.; Montgomery Jr., J. A.; Peralta, J. E.; Ogliaro, F.; Bearpark, M. J.; Heyd, J. J.; Brothers, E. N.; Kudin, K. N.; Staroverov, V. N.; Keith, T. A.; Kobayashi, R.; Normand, J.; Raghavachari, K.; Rendell, A. P.; Burant, J. C.; Iyengar, S. S.; Tomasi, J.; Cossi, M.; Millam, J. M.; Klene, M.; Adamo, C.; Cammi, R.; Ochterski, J. W.; Martin, R. L.; Morokuma, K.; Farkas, O.; Foresman, J. B.; Fox, D. J. Gaussian 16 Rev. A.03, Wallingford, CT (2016).

(4) Becke, A. D. Density-functional thermochemistry. III. The role of exact exchange. J. Chem. Phys. 1993, 98, 5648.

(5) Kim, K.; Jordan, K. D. Comparison of Density Functional and MP2 Calculations on the Water Monomer and Dimer. J. Chem. Phys. 1994, 98, 10089.

(6) Stephens, P. J.; Devlin, F. J.; Chabalowski, C. F.; Frisch, M. J. Ab Initio Calculation of Vibrational Absorption and Circular Dichroism Spectra Using Density Functional Force Fields. J. Chem. Phys. 1994, 98, 11623.

(7) Weigend, F.; Ahlrichs, R. Balanced basis sets of split valence, triple zeta valence and quadruple zeta valence quality for $\mathrm{H}$ to $\mathrm{Rn}$ : Design and assessment of accuracy. Phys. Chem. Chem. Phys. 2005, 7, 3297.

(8) Petersson, G. A.; Al-Laham, M. A. A complete basis set model chemistry. II. Open-shell systems and the total energies of the first-row atoms. J. Chem. Phys. 1991, 94, 6081.

(9) Petersson, G. A.; Bennett, A.; Tensfeldt, T. G.; Al-Laham, M. A.; Shirley, W. A.; Mantzaris, J. A complete basis set model chemistry. I. The total energies of closed-shell atoms and hydrides of the first-row elements. J. Chem. Phys. 1988, 89, 2193.

(10) Binning Jr, R. C.; Curtiss, L. A. Compact contracted basis sets for third-row atoms: Ga-Kr. J. Comput. Chem. 1990, 11, 1206.

(11) Blaudeau, J.-P.; McGrath, M. P.; Curtiss, L. A.; Radom, L. Extension of Gaussian-2 (G2) theory to molecules containing third-row atoms K and Ca. J. Chem. Phys. 1997, 107, 5016.

(12) Curtiss, L. A.; McGrath, M. P.; Blaudeau, J. P.; Davis, N. E.; Binning, R. C.; Radom, L. Extension of Gaussian-2 theory to molecules containing third-row atoms Ga-Kr. J. Chem. Phys. 1995, 103, 6104.

(13) Hay, P. J. Gaussian basis sets for molecular calculations. The representation of $3 \mathrm{~d}$ orbitals in transition-metal atoms. J. Chem. Phys. 1977, 66, 4377.

(14) Krishnan, R.; Binkley, J. S.; Seeger, R.; Pople, J. A. Self-consistent molecular orbital methods. XX. A basis set for correlated wave functions. J. Chem. Phys. 1980, 72, 650. 
(15) McGrath, M. P.; Radom, L. Extension of Gaussian-1 (G1) theory to bromine-containing molecules. J. Chem. Phys. 1991, 94, 511.

(16) McLean, A. D.; Chandler, G. S. Contracted Gaussian basis sets for molecular calculations. I. Second row atoms, Z=11-18. J. Chem. Phys. 1980, 72, 5639.

(17) Raghavachari, K.; Trucks, G. W. Highly correlated systems. Excitation energies of first row transition metals Sc-Cu. J. Chem. Phys. 1989, 91, 1062.

(18) Wachters, A. J. H. Gaussian Basis Set for Molecular Wavefunctions Containing Third-Row Atoms. J. Chem. Phys. 1970, 52, 1033.

(19) Grimme, S.; Antony, J.; Ehrlich, S.; Krieg, H. A consistent and accurate ab initio parametrization of density functional dispersion correction (DFT-D) for the 94 elements HPu. J. Chem. Phys. 2010, 132, 154104. 